Japan. J. Math.

Vol. 17, No. 2, 1991

\title{
Amalgams of quasithin solvable groups
}

\author{
By Yasuhiko TANaKa \\ (Received May 7, 1990)
}

\section{$\S 1 . \quad$ Introduction}

In this paper we study the triples $(X, S, Y)$ of finite groups satisfying the following conditions:

(a) $\quad S$ is a common Sylow 2-subgroup of $X$ and $Y$;

(b) both $X$ and $Y$ are 2-irreducible;

(c) no nonidentity subgroup of $S$ is normal both in $X$ and in $Y$;

(d) $\quad C_{X}\left(O_{2}(X)\right) \subseteq O_{2}(X)$ and $C_{Y}\left(O_{2}(Y)\right) \subseteq O_{2}(Y)$.

Here, a group $G$ is said to be $H$-irreducible for a subgroup $H$ of $G$ if $H$ is contained in a unique maximal subgroup of $G$, and a finite group $G$ is said to be 2-irreducible if $G$ is $T$-irreducible for a Sylow 2-subgroup $T$ of $G$. We will call the triple $(X, S, Y)$ an amalgam because we often embed the groups $S, X, Y$ into the amalgamated product of $X$ and $Y$ over $S$.

In previous papers [7] and [11], we studied such amalgams of thin solvable groups, and determined the isomorphism types (or the isomorphism classes) of possible ones. Applying the result, Gomi [3] gave an alternative short proof of Janko's theorem [9] which classifies the finite thin simple groups with solvable 2local subgroups. The success has stimulated our study to obtain a similar alternative proof of Smith's theorem [10] which classifies the finite quasithin simple groups with solvable 2-local subgroups.

The purpose of this paper is to lay a foundation for such a proof by extending the result of [7] to amalgams of quasithin solvable groups. In fact, in their forthcoming paper $[\mathbf{6}]$, Gomi and Hayashi will apply our result to an alternative proof of Smith's theorem.

In order to state our result precisely, we review here the following definition.

A finite group $G$ is said to be thin (resp. quasithin) if $e(G) \leqq 1$ (resp. $e(G) \leqq 2$ ), where the invariant $e(G)$ is defined as follows:

$e(G)=\max \left\{m_{2, p}(G) \mid 2 \neq p \in \pi(G)\right\}$

$m_{2, p}(G)=\max \left\{m_{p}(L) \mid L\right.$ is a 2-local subgroup of $\left.G\right\}$;

$m_{p}(L)=\max \left\{m_{p}(E) \mid E\right.$ is an elementary abelian $p$-subgroup of $\left.L\right\}$.

In this terminology, the main result of [7] may be stated as follows. 
Theorem A $([\mathbf{7}])$. Let $S, X, Y$ be finite groups satisfying the conditions (a)(d) and the following additional condition $\left(\mathrm{e}_{1}\right)$ :

$\left(\mathrm{e}_{1}\right)$ both $X$ and $Y$ are solvable and thin.

Then the amalgam $(X, S, Y)$ or $(Y, S, X)$ is a $G L_{3}(2)$-amalgam, an $S p_{4}(2)$ amalgam, a $G_{2}(2)^{\prime}$-amalgam, a $G_{2}(2)$-amalgam, an $M_{12}$-amalgam, an $\operatorname{Aut}\left(M_{12}\right)$ amalgam, a ${ }^{2} F_{4}(2)^{\prime}$-amalgam, or $a^{2} F_{4}(2)$-amalgam.

We will give no precise definition of the above eight types of amalgams, but we will only note that the isomorphism classes of $S, X, Y$ are completely determined. (See $[\mathbf{7}]$ and $[\mathbf{1 1}]$ for details.)

Now, our result may be stated as follows.

Theorem B. Let $S, X, Y$ be finite groups satisfying the conditions (a)-(d) and the following additional conditions $\left(\mathrm{e}_{2}\right)$ and $(\mathrm{g})$ :

$\left(\mathrm{e}_{2}\right)$ both $X$ and $Y$ are solvable and quasithin;

(g) $S=\left[O_{2}(X), O^{2}(X)\right]\left(O_{2}(X) \cap O_{2}(Y)\right)\left[O_{2}(Y), O^{2}(Y)\right]$. Then both $X$ and $Y$ are thin.

We note that Theorem B together with Theorem A determines the isomorphism classes of $S, X, Y$ for all amalgams $(X, S, Y)$ satisfying the conditions (a)-(d), $\left(\mathrm{e}_{2}\right)$, and $(\mathrm{g})$. Also, Theorem B really generalizes Theorem A because the amalgams considered in [7] satisfy the condition $(\mathrm{g})$.

Though the invariant $e(G)$ for a finite group $G$ has been playing an important role in the finite group theory, the new invariant $f(G)$ defined as follows seems more essential than $e(G)$ to the analysis both in $[6]$ and in this paper:

$$
\begin{aligned}
& f(G)=\max \left\{n_{2, p}(G) \mid 2 \neq p \in \pi(G)\right\} ; \\
& n_{2, p}(G)=\max \left\{n_{p}(L) \mid L \text { is a } 2 \text {-local subgroup of } G\right\} ; \\
& n_{p}(L)=\max \left\{m_{p}(V) \mid V \text { is a chief } p \text {-factor of } L\right\} .
\end{aligned}
$$

Indeed, it is not Theorem $\mathrm{B}$ but the following theorem that is proved in this paper and applied to [6].

Theorem C. Let $S, X, Y$ be finite groups satisfying the conditions (a)-(d), $(\mathrm{g})$, and the following condition $\left(\mathrm{f}_{2}\right)$ :

$\left(f_{2}\right)$ both $X$ and $Y$ are solvable and $f(X) \leqq 2 \geqq f(Y)$. Then both $X$ and $Y$ are thin.

Since Thompson observed in his analysis in the $N$-group paper [12] that $e(G) \leqq 2$ implies $f(G) \leqq 2$, Theorem $\mathrm{C}$ is stronger than Theorem B. Moreover, since $[6]$ needs the structure of only the amalgams with the conditions $(a)-(d),\left(f_{2}\right)$, and $(\mathrm{g})$, our study is sufficient to an application to Smith's theorem.

It may be possible to generalize Theorem $\mathrm{C}$ further by eliminating the condition $(\mathrm{g})$, but such a generalization does not seem to have important applications. 
On the other hand, it is no doubt relevant to replace the condition $\left(f_{2}\right)$ by the following weaker condition (f):

(f) both $X$ and $Y$ are solvable.

In fact, this kind of generalization is necessary to a similar alternative proof of a theorem of Gorenstein and Lyons [8] which completes the classification of the finite simple groups with solvable 2-local subgroups.

Theorem $\mathrm{C}$ is established by a graph theoretical approach of Delgado, Goldshmidt, and Stellmacher [2]. For an amalgam $(X, S, Y)$, we construct the amalgamated product $G$ of $X$ and $Y$ over $S$, and define $\Gamma=\Gamma(X, S, Y)$ to be the (right) coset graph of $G$ with respect to the subgroups $X$ and $Y$. Then $G$ acts on $\Gamma$ by (right) multiplication. For a vertex $\xi$ of $\Gamma$, we denote by $G_{\xi}$ the stabilizer of $\xi$ in $G$, and put $Q_{\xi}=O_{2}\left(G_{\xi}\right)$ and $V_{\xi}=\left\langle\Omega_{1}(Z(T)) \mid T \in \operatorname{Syl}_{2}\left(G_{\xi}\right)\right\rangle$. Finally, we set $b(X, S, Y)$ to be the minimum distance $d(\alpha, \delta)$ of $\alpha, \delta \in V(\Gamma)$ under the condition that $V_{\alpha} \nsubseteq Q_{\delta}$.

Suppose that the amalgam $(X, S, Y)$ satisfies the hypothesis of Theorem C. We divide the proof of Theorem $\mathrm{C}$ into three parts by the value of $b=b(X, S, Y)$ : the small case; the even case; the odd case. First, we deal with the small case where $b \leqq 2$ in Section 3 . In this case, we can replace the condition $\left(\mathrm{f}_{2}\right)$ by the weaker condition (f). Next, we treat the case where $b$ is even and $b \geqq 4$ in Section 5 , and the case where $b$ is odd and $\mathrm{b} \geqq 3$ in Sections $6-8$. Contrary to the small case, our present proof needs the condition $\left(f_{2}\right)$ in the latter two cases.

Since most of the analysis of this paper is devoted to determination of the chief 2 -factors involved within $X$ and $Y$, the theory of $G F(2)$-representations for 2 -irreducible solvable groups is of fundamental importance. The necessary lemmas on $G F(2)$-representations for such groups are collected in the next section.

We close this section with the following four lemmas.

(1.1) Let $S$ be a 2-group, and $t$ a noncentral involution of $S$. Then the following holds.

(1) There exists $u \in t^{S}$ such that $\langle t, u\rangle \cong \mathbf{E}_{2^{2}}$.

(2) If $m(S)=2$, then $\Omega_{1}(Z(S)) \subseteq\langle t, u\rangle$ and $\langle t, u\rangle$ is the unique fours subgroup of $S$ containing $t$.

Proof. Take $s \in N_{S}\left(C_{S}(t)\right)-C_{S}(t)$, and put $u=t^{s}$. Then $u$ has the desired property. Suppose $m(S)=2$, and let $t \in E \subseteq S$ and $E \cong \mathbf{E}_{2^{2}}$. Then $\Omega_{1}(Z(S)) \subseteq$ $E$ and so $E=\left\langle t, \Omega_{1}(Z(S))\right\rangle$. Thus $E$ is the unique fours subgroup of $S$ containing $t$.

(1.2) Let $t$ be an involution inverting an abelian group $P$ of odd order, and $V$ a $G F(2) P\langle t\rangle$-module with $C_{V}(P)=0$. Then $|V|=\left|V: C_{V}(t)\right|^{2}$.

Proof. We may assume that $V$ is faithful and indecomposable. Let $C$ be 
a nonidentity cyclic subgroup of $P$, and take $u \in t^{C}$ so that $C=\langle t u\rangle$. Then, by the $P\langle t\rangle$-invariant decomposition $V=[V, C] \oplus C_{V}(C)$, we have $C_{V}(t) \cap C_{V}(u)$ $\subseteq C_{V}(C)=0$, and hence $|V|=\left|V: C_{V}(t)\right|^{2}$.

(1.3) Let $G$ be a finite group acting on a normal series

$$
T=T_{0} \supseteqq T_{1} \supseteqq \cdots \supseteqq T_{r-1} \supseteqq T_{r}=1
$$

of a finite group $T$. Then

$$
|T: U| \cdot|[U, A]| \geqq \prod_{i=1}^{r}\left|T_{i-1} / T_{i}: U_{i} / T_{i}\right| \cdot\left|\left[U_{i} / T_{i}, A\right]\right|
$$

for a subgroup $A$ of $G$ and an $A$-invariant subgroup $U$ of $T$, where $U_{i}=\left(T_{i-1} \cap\right.$ $U) T_{i}(1 \leqq i \leqq r)$.

Proof. Define $V_{i}=\left(T_{i-1} \cap[U, A]\right) T_{i}(1 \leqq i \leqq r)$. Then we have

$$
\begin{aligned}
& {\left[U_{i} / T_{i}, A\right] \subseteq V_{i} / T_{i}(1 \leqq i \leqq r), \text { and }} \\
& |T: U| \cdot|[U, A]|=\prod_{i=1}^{r}\left|T_{i-1} / T_{i}: U_{i} / T_{i}\right| \cdot\left|V_{i} / T_{i}\right| .
\end{aligned}
$$

Hence the assertion follows.

(1.4) Let $P$ be a p-group for some odd prime $p$ acting on a 2-group $T$. Suppose that $P=P_{1} P_{2} \triangleright P_{i}(i=1,2)$, that $T=C_{T}\left(P_{1}\right) C_{T}\left(P_{2}\right)$, and that $C_{T}(P)=1$. Then

$$
T=C_{T}\left(P_{1}\right) \times C_{T}\left(P_{2}\right) .
$$

Proof. It suffices to prove that $C_{T}\left(P_{i}\right) \triangleleft T(i=1,2)$. Let $\{i, j\}=\{1,2\}$. Then, since $P_{j}$ acts on $C_{T}\left(P_{i}\right)$, we have

$$
C_{T}\left(P_{i}\right)=\left[C_{T}\left(P_{i}\right), P_{j}\right] C_{C_{T}\left(P_{i}\right)}\left(P_{j}\right)=\left[C_{T}\left(P_{i}\right) C_{T}\left(P_{j}\right), P_{j}\right] C_{T}(P)=\left[T, P_{j}\right] \triangleleft T .
$$

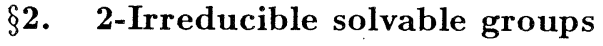

Throughout this section, we will assume that all groups are finite and that all $G F(2)$-representations of groups are finite dimensional. This section is devoted to a preliminary study of 2 -irreducible solvable groups.

The first lemma describes the fundamental structure of such groups. Recall that $n_{p}(G)=\max \left\{m_{p}(V) \mid V\right.$ is a chief $p$-factor of $\left.G\right\}$ for a group $G$ and an odd prime $p$. 
Lemma 2.1 Let $G$ be a 2-irreducible solvable group, $S$ a Sylow 2-subgroup of $G$, and $M$ the unique maximal subgroup of $G$ containing $S$. Put $Q=\mathrm{O}_{2}(G)$. Then the following holds.

(1) $O^{2}(G) \neq 1$.

(2) If $N \triangleleft G \neq S N$ (or, equivalently, $O^{2}(G) \nsubseteq N \triangleleft G$ ), then $S \cap N \triangleleft G$, $Q \in \operatorname{Syl}_{2}(Q N), O_{2}(G / N)=Q N / N$, and $G / N$ is 2-irreducible and solvable.

(3) $G=O_{2, p, 2}(G)$ for some odd prime $p$.

Let $P \in \operatorname{Syl}_{p}(G)$.

(4) $S / Q$ acts irreducibly and faithfully on $P Q / \Phi(P) Q$.

(5) $Z(S / Q)$ is cyclic, and an element $t$ of $S$ inverts $P Q / \Phi(P) Q$ if and only if $Q \neq t Q \in \Omega_{1}(Z(S / Q))$.

(6) If $n_{p}(G) \leqq 2$, then $m_{p}(P / \Phi(P)) \leqq 2$ and $m_{2}(S / Q) \leqq 2$.

(7) The following holds:

(i) $P$ is cyclic if and only if $n_{p}(G)=1$;

(ii) if $S / Q$ is elementary abelian, then $|S / Q| \leqq 2$ and $P$ is cyclic;

(iii) if $P$ is cyclic, then $S / Q$ is cyclic.

(8) $\quad M=\Phi(P) S, \cap M^{G}=\Phi(P) Q$, and $\Phi(P) Q / Q=\Phi\left(O^{2}(G / Q)\right)$.

(9) If $O^{2}(G / Q N)$ is cyclic and nonidentity (or, equivalently, $n_{p}(G / Q N)=$ 1) for some $N \triangleleft G$, then $P$ is cyclic and $n_{p}(G)=1$.

(10) If $Q \neq z Q \in \Omega_{1}(Z(S / Q))$ for an element $z \in S$, then the following four conditions for an element $x \in G$ are equivalent:

(i) $x \notin M$;

(ii) $\quad G=\left\langle S, z^{x}\right\rangle$;

(iii) $\quad G=\left\langle S, S^{x}\right\rangle$;

(iv) $\quad G=\langle S, x\rangle$.

(11) If $x \in S-Q$, there exists $T \in \operatorname{Syl}_{2}(G)$ such that $G=\langle T, x\rangle$.

Proof. Part (1) holds because $S \subseteq M \subset G$.

Let $N \triangleleft G \neq S N$. Then $G=N_{G}(S \cap N) S N$ by a Frattini argument. Thus the 2-irreducibility of $G$ shows that $G=N_{G}(S \cap N)$, and hence $S \cap N \triangleleft G$. Since $Q N \triangleleft G \neq S Q N$, we also have $Q \subseteq S \cap Q N \triangleleft G$, so $Q=S \cap Q N \in \operatorname{Syl}_{2}(Q N)$. Let $\bar{G}=G / N$. Then $\bar{S} \in \operatorname{Syl}_{2}(\bar{G})$ and $\bar{M}$ is the unique maximal subgroup of $\bar{G}$ containing $\bar{S}$. Suppose $R$ is the inverse image of $O_{2}(\bar{G})$ in $G$. Then $Q N \subseteq R$ $\subseteq S N$, so $Q=S \cap R \in \operatorname{Syl}_{2}(R)$ as above. Thus the index $|R: Q N|$ is odd, and hence $R=Q N$. This proves (2).

For the remainder of this proof, we may assume $Q=1$, replacing $G$ by $G / Q$.

The solvability of $G$ shows that either $G=O(G)$ or $O_{2}(G / O(G)) \neq 1$, so $G=S O(G)$ by (2). Therefore $G=O_{2^{\prime}, 2}(G)$. Let $p$ be a prime divisor of the index $|G: M|$, and choose a Hall $\{2, p\}$-subgroup, say $H$, of $G$ containing $S$. Then we have $H \nsubseteq M$, and so $G=H$ by the 2-irreducibility of $G$. Thus (3) holds.

Now, let $P \in \operatorname{Syl}_{p}(G)$ and $W=P / \Phi(P)$. Then $P=O_{p}(G)$ by (3), and so 
$\Phi(P) \triangleleft G$ and $S$ acts faithfully on $W$. Since $W$ is completely reducible as a $G F(p) S$ module by Maschke's theorem, the 2-irreducibility of $G$ forces that $S$ is irreducible on $W$. This proves (4), and hence $Z(S)$ is cyclic and $\Omega_{1}(Z(S))$ inverts $W$ by Schur's lemma. Put $\Omega_{1}(Z(S))=\langle z\rangle$, and suppose $W$ is inverted by some element $t \in S$. Then $t z \in C_{S}(W)=1$, and so $t=z$. Thus (5) holds.

In proving (6) and (7), let $m=m_{p}(W)$. Then we have $1 \leqq m \leqq n_{p}(G)$ because $P \neq 1$ by (1) and $W$ is a chief factor of $G$ by (3) and (4). Since $S$ is isomorphic to an irreducible subgroup of Aut $(W)\left(\cong G L_{m}(p)\right)$ by $(4)$, we conclude that $m_{2}(S) \leqq 2$ if $n_{p}(G) \leqq 2$, and that $S$ is cyclic if $m=1$. This proves (6) and (iii) of (7). Part (i) of (7) holds because $P \triangleleft G$ by (3), and (ii) of (7) holds by (4) and (5).

Since $P=O^{2}(G)$ by $(3)$, we have $\Phi(P)=\Phi\left(O^{2}(G)\right)$. Put $L=\cap M^{G}$. Let $N \triangleleft$ $G$, and suppose $O^{2}(G / N) \neq 1$. Then we have $G \neq S N$, so $N$ has odd order by (2). Thus $L \subseteq P$ by setting $N=L$, and so $N \cong L \subseteq P \cap M \neq P$. Therefore we have $\Phi(P)=L=P \cap M$ by setting $N=\Phi(P)$, and hence $N \subseteq \Phi(P)$ and $M=S \Phi(P)$. This proves (8). Suppose that $O^{2}(G / Q N)$ is cyclic and nonidentity. Since $G / N$ is 2-irreducible and solvable with $O_{2}(G / N)=1$ by $(2)$, we have $O^{2}(G / N)=P / N$ by (3). Hence $P$ is cyclic as $N \subseteq \Phi(P)$ by (8), and so $n_{p}(G)=1$ by (7). Thus (9) holds.

To prove (10), first let $x \notin M$. Put $\bar{G}=G / \Phi(P)$, and take an element $y \in P$ so that $\bar{x} \in \overline{S y}$. Since $\bar{z}$ inverts $\bar{y}$ by (4), $\bar{y} \in\left\langle\bar{z}, \bar{z}^{\bar{y}}\right\rangle \subseteq\left\langle\bar{S}, \bar{z}^{\bar{y}}\right\rangle=\left\langle\bar{S}, \bar{z}^{\bar{x}}\right\rangle$, so $M \not \ngtr y \in\left\langle S, z^{x}\right\rangle \Phi(P)$. Therefore $\left\langle S, z^{x}\right\rangle \nsubseteq M$, and hence $G=\left\langle S, z^{x}\right\rangle$. This shows that (i) implies (ii). Clearly, (ii) implies (iii), (iii) implies (iv), and (iv) implies (i).

Finally, let $1 \neq x \in S$. Since $x \notin \Phi(P)=\cap M^{G}$ by (8), there exists an element $g \in G$ such that $x \notin M^{g}$. Thus we have $G=\left\langle S^{g}, x\right\rangle$ by (10). This proves (11).

In the next two propositions, we need the following definitions.

Let $S$ be a 2 -group. We will denote by $\mathcal{A}(S)$ the set of elementary abelian subgroups of $S$ of maximal order. Put $J(S)=\langle\mathcal{A}(S)\rangle$, the Thompson subgroup of $S$, and $K(S)=C_{S}\left(\Omega_{1}(Z(J(S)))\right)$. The characteristic subgroup $Q(S)$ of $S$ is defined in $[5]$.

Proposition 2.2 Let $G$ be a 2 -irreducible solvable group with $C_{G}\left(O_{2}(G)\right) \subseteq$ $\mathrm{O}_{2}(G)$, and $S$ a Sylow 2-subgroup of $G$. Suppose that $\Omega_{1}(Z(S)) \varsubsetneqq Z(G)$ and that $Q(K(S)) \nless G$. Then

$$
\left[O_{2}(G), O^{2}(G)\right] \subseteq\left\langle\Omega_{1}(Z(S))^{G}\right\rangle
$$

Proof. Let $Z=\Omega_{1}(Z(S))$ and $V=\Omega_{1}\left(Z\left(O_{2}(G)\right)\right)$. Then we have $Z \subseteq V$ because $C_{G}\left(O_{2}(G)\right) \subseteq O_{2}(G)$. Put $\bar{G}=G / C_{G}(V)$. We will regard $V$ as a faithful $G F(2) \bar{G}$-module.

Since $G$ satisfies Hypothesis III of [4], the pair $(\bar{G}, V)$ satisfies Hypothesis II of [4] by 6.6 of [4]. Therefore $5.1,5.3$, and 5.4 of [4] force $G$ to have a unique noncentral 
chief factor within $V$, and then it lies within $\left\langle Z^{G}\right\rangle$ because $Z(G) \nsupseteq Z \subseteq V$. Since $\left[O_{2}(G), O^{2}(G)\right] \subseteq V$ by 6.7 of $[4]$, we conclude that $\left[O_{2}(G), O^{2}(G)\right] \subseteq\left\langle Z^{G}\right\rangle$.

Proposition 2.3 Let $G$ be a 2-irreducible solvable group with $C_{G}\left(O_{2}(G)\right) \subseteq$ $\mathrm{O}_{2}(G), S$ a Sylow 2-subgroup of $G$, and $A$ a group of automorphisms of $S$ of odd order. Then some nonidentity A-invariant subgroup of $S$ is normal in $G$.

Proof. Suppose false. Then no nonidentity characteristic subgroup of $S$ is normal in $G$. Thus, by the corollary of the main theorem of $[\mathbf{4}], G$ is described as follows:

$$
\begin{aligned}
& G=S N \\
& N=\left\langle J(S)^{G}\right\rangle=L_{1} \times \cdots \times L_{m} \times D_{1} \times \cdots \times D_{n} \times E \\
& L_{1} \cong \cdots \cong L_{m} \cong \Sigma_{4} \\
& D_{1} \cong \cdots \cong D_{n} \cong \mathbf{D}_{8} \\
& E^{2}=1
\end{aligned}
$$

Let $T=J(S)$. Since $T \cong S \cap N=J(S \cap N)$ by the structure of $N$, we have $T=S \cap N \in \operatorname{Syl}_{2}(N)$. Now, define $Q=O_{2}(N), T_{i}=T \cap L_{i}, Z_{i}=T_{i}^{\prime}$, $V_{i}=O_{2}\left(L_{i}\right), \mathbf{E}_{2^{2}} \cong W_{i} \leqq T_{i}=\left\langle V_{i}, W_{i}\right\rangle(i \leqq i \leqq m), Z=Z_{1} \times \cdots \times Z_{m}$, $V=V_{1} \times \cdots \times V_{m}, W=W_{1} \times \cdots \times W_{m}$. Then the Krull-Remak-Schmidt theorem together with the 2-irreducibility of $G$ yields that $S$ permutes, by conjugation, $Z_{1}, \ldots, Z_{m}, D_{1}^{\prime}, \ldots, D_{n}^{\prime}$, and transitively permutes $L_{1}^{\prime}, \ldots, L_{m}^{\prime}$. Thus we conclude that $S$ transitively permutes $Z_{1}, \ldots, Z_{m}$. Since $\left[Q, O^{2}(G)\right] \subseteq V$, we can take, by our hypothesis, $a \in A$ so that $T=T^{a} \supseteqq V^{a} \varsubsetneqq Q=C_{T}(V)$. Since $S \triangleright\left[V, V^{a}\right]=$ $\left[V_{1}, V^{a}\right] \times \cdots \times\left[V_{m}, V^{a}\right]$ and $\left[V_{i}, V^{a}\right]=1$ or $Z_{i}(1 \leqq i \leqq m)$, we have $\left[V, V^{a}\right]=Z$ by the choice of $a$ and the above remark. Therefore $\left[V^{a^{-1}}, V\right]=Z^{a^{-1}} \neq 1$, and hence $V^{a^{-1}} \nsubseteq Q$. Thus the same argument also shows that $\left[V, V^{a^{-1}}\right]=Z$, so we have

$$
Z=\left[V, V^{a}\right]=Z^{a} .
$$

Let $\mathcal{A}=\mathcal{A}(S)$, and put

$$
\mathcal{A}_{V}=\{B \in \mathcal{A} \mid V \subseteq B\} \quad \text { and } \quad \mathcal{A}_{W}=\{B \in \mathcal{A} \mid W \subseteq B\}
$$

Let $B \in \mathcal{A}$. Then we have

$$
B \cap T_{i}=V_{i} \text { or } W_{i} \text { for } 1 \leqq i \leqq m
$$

because $B=\left(B \cap T_{1}\right) \times \cdots \times\left(B \cap T_{m}\right) \times\left(B \cap\left(D_{1} \times \cdots D_{n} \times E\right)\right)$. Therefore

$$
\mathcal{A}_{V} \cap \mathcal{A}_{W}=\phi
$$

and, for $B, C \in \mathcal{A}$ with $[B, C] \supseteqq Z$, we have $B \in \mathcal{A}_{V}$ if and only if $C \in \mathcal{A}_{W}$. 
Now, let $s=|\langle a\rangle|$. Pick $B \in \mathcal{A}_{V}$, and define $B_{k}=B^{a^{k}}(k \geqq 0)$. Then $B_{k} \in \mathcal{A}$ and $\left[B_{k}, B_{k+1}\right] \supseteqq\left[V^{a^{k}}, V^{a^{k+1}}\right]=\left[V, V^{a}\right]^{a^{k}}=Z^{a^{k}}=Z(k \geqq 0)$, so

$$
B_{0}=B \in \mathcal{A}_{V}, B_{1} \in \mathcal{A}_{W}, \ldots, B_{s-1} \in \mathcal{A}_{V}, B=B_{s} \in \mathcal{A}_{W}
$$

by the above remark. But this contradicts that $\mathcal{A}_{V} \cap \mathcal{A}_{W}=\phi$.

Let $G$ be a 2-irreducible solvable group with $C_{G}\left(O_{2}(G)\right) \subseteq O_{2}(G)$, and let $V$ be a noncentral chief 2 -factor of $G$. Then the pair $(G, V)$ satisfies the hypothesis of the following lemma, regarding $V$ as a $G F(2) G$-module.

Lemma 2.4 Let $G$ be a 2-irreducible solvable group, $S$ a Sylow 2-subgroup of $G$, and $V$ a $G F(2) G$-module with $Q=O_{2}(G) \subseteq C_{G}(V) \nsupseteq O^{2}(G)$. Put $\bar{G}=$ $G / C_{G}(V)$. Then the following holds.

(1) $\bar{G}$ is a 2-irreducible solvable group with $O_{2}(\bar{G})=1, C_{S}(V)=Q$, and $C_{G}(V) / Q \subseteq \Phi\left(O^{2}(G / Q)\right)$.

(2) $C_{G}\left(\left[V, O^{2}(G)\right]\right)=C_{G}(V)$.

(3) $\quad V=\left[V, O^{2}(G)\right] \oplus C_{V}\left(O^{2}(G)\right)$.

Proof. Part (1) follows from (2.1)-(2)(8). For the proof of (2) and (3), put $P=O^{2}(G)$ and $W=[V, P]$. Note that $O^{2}(\bar{G})=\bar{P}$. We may assume $C_{G}(V)=1$ replacing $G$ by $\bar{G}$ if necessary. Since $[W, P]=W \neq 0$, we have $P \nsubseteq C_{G}(W)$, and hence $C_{G}(W)$ has odd order by $(2.1)-(2)$. Thus $C_{G}(W) \subseteq O^{2}\left(C_{G}(V / W / 1)\right) \subseteq C_{G}(V)=$ 1 , so (2) holds. Part (3) holds because $P$ has odd order by (2.1)-(3).

From now on, we will use the following terminology and notation.

First, let $G$ and $H$ be groups. We will say that a $G F(2) G$-module $V$ is induced by a $G F(2) H$-module $W$ and denote by $(G, V) \ll(H, W)$ if there exist a one to one homomorphism $\sigma: \bar{G}=G / C_{G}(V) \longrightarrow \bar{H}=H / C_{H}(W)$ and a $G F(2)$-isomorphism $f: V \longrightarrow W$ such that $(v \bar{g}) f=(v f)(\bar{g} \sigma)$ for all $v \in V$ and $g \in G$.

Next, let $G$ be a group acting on a group $T$. Then we will define

$$
\begin{aligned}
& {[T, G ; 0]=T, \quad \text { and }} \\
& {[T, G ; k]=[[T, G ; k-1], G] \text { for } \quad k \geqq 1 .}
\end{aligned}
$$

In order to clarify the structure of chief 2 -factors of 2 -irreducible solvable groups, we often need several information on faithful $G F(2) \vec{G}$-modules for 2-irreducible solvable groups $\bar{G}$ with $O_{2}(\bar{G})=1$ by $(2.4)$. Thus, for a while, we will study such $G F(2)$-representations.

The first three lemmas give precise information on certain $G F(2)$-representations for 2-irreducible $\{2,3\}$-groups with Sylow 3-subgroups isomorphic to $\mathbf{E}_{3^{2}}$ or $E s p_{27}$. Here, $E s p_{27}$ denotes the extraspecial group of order 27 and of exponent 3 . 
Lemma 2.5 Let $G$ be a 2-irreducible $\{2,3\}$-group with $O_{2}(G)=1, S \in \operatorname{Syl}_{2}(G)$, $P=O^{2}(G) \cong \mathbf{E}_{3^{2}}$, and $V$ a four dimensional faithful $G F(2) G$-module. Define

$$
V_{n}=[V, S ; n](n \geqq 0), Z=\Omega_{1}(Z(S)) \text {, and } E=C_{S}\left(V_{2}\right) .
$$

Then the following holds.

(1) $(G, V) \ll\left(O_{4}^{+}(2), G F(2)^{4}\right), S \cong \mathbf{Z}_{4}$ or $\mathbf{D}_{8},|S: E|=2$, and $Z \cong E$.

(2) If $S \cong \mathbf{D}_{8}$ (or, equivalently, $G \cong O_{4}^{+}(2)$ ), then there exist generators $\sigma$, $\tau$ of $S$, generators $\varphi, \psi$ of $P$, and a basis $\left\{v_{1}, v_{2}, v_{3}, v_{4}\right\}$ of $V$ such that

$$
\left\{\begin{array}{l}
v_{1}^{\sigma}=v_{4}, v_{2}^{\sigma}=v_{2}, v_{3}^{\sigma}=v_{3}, \\
v_{1}^{\tau}=v_{2}, v_{3}^{\tau}=v_{4}, \\
v_{1}^{\varphi}=v_{4}, v_{2}^{\varphi}=v_{2}, v_{3}^{\varphi}=v_{3}, v_{4}^{\varphi}=v_{1}+v_{4} \\
v_{1}^{\psi}=v_{1}, v_{2}^{\psi}=v_{3}, v_{3}^{\psi}=v_{2}+v_{3}, v_{4}^{\psi}=v_{4} .
\end{array}\right.
$$

(3) $\left|V_{n-1}: V_{n}\right|=2(1 \leqq n \leqq 4)$, and

$$
V=V_{0} \supseteqq V_{1} \supseteqq V_{2} \supseteqq V_{3} \supseteqq V_{4}=0
$$

is the unique $S$-composition series of $V$.

(4) $V_{3}=C_{V}(S), V_{2}=[V, T]=C_{V}(T)$ for $T=Z$ or $E$, and $E$ is the unique quadratic fours subgroup of $S$ if $S \cong \mathbf{D}_{8}$.

Let $A$ be an elementary abelian subgroup of $S$.

(5) If $\left|V: C_{V}(A)\right| \leqq|A|$ or $|[V, A]| \leqq|A|$, then $Z \neq A \subseteq E$ and $\mid V$ : $C_{V}(A)|=| A|=|[V, A] \mid$.

(6) If $Z \neq A \leqq E$ and $A \subseteq B \leqq C_{S}(A)$, then $[V, B]=[V, A]+$ $\left[C_{V}(A), B\right]$.

(7) If $1 \neq A \subseteq E$, then $V_{2}=V_{3}+[V, A]=C_{V_{1}}(A)$.

(8) Let $V_{2} \subset W \subset V$. If $1 \neq A \subseteq E$, then the following holds:

(i) if $[W, A]=V_{2}$, then $Z \neq A=E$ and $W=V_{1}$;

(ii) if $[W, A]=V_{3}$, then $A=Z$ and $W=V_{1}$;

(iii) if $[W, A]=0$, then $Z \neq A \neq E$ and $W \neq V_{1}$.

Let $T \subseteq S$.

(9) If $T \neq 1$, then $[V, T] \nsubseteq V_{3}$ and $\left[V_{1}, T\right] \neq 0$.

(10) Suppose $\left|V: C_{V}(T)\right| \leqq 2^{2}$. Then $|[V, T]| \leqq 2^{2}$ and, moreover, $[V, T]$ $\supseteq C_{V}(S)$ if $|[V, T]|=2^{2}$.

Suppose $S \cong \mathbf{D}_{8}$.

(11) Let $H=E P$ and $x \in G$. Choose $y \in P$ so that $x \in S y$. Then the following five conditions are equivalent:

(i) $E \cap E^{x} \neq 1$;

(ii) $H \neq\left\langle E, E^{x}\right\rangle$; 
(iii) $\langle y\rangle \triangleleft H$;

(iv) $\quad V_{2} \cap V_{2}{ }^{x} \neq 0$;

(v) $C_{V}(y) \neq 0$.

(12) Let $x \in G$. Then $S \cap S^{x} \neq 1$ and $E \cap E^{x}=E \cap S^{x}=S \cap E^{x}$.

(13) Let $x \in G$. Then $S \cap S^{x} \neq 1$ and $E \cap E^{x}=E \cap S^{x}=S \cap E^{x}$.

Proof. Embed $G=S P$ into $L=G L(V)$, and let $N=N_{L}(P)$. Then $G \cong N \cong O_{4}^{+}(2)$ and $N$ is a Sylow 3-normalizer of $L$. Choose $R \in \operatorname{Syl}_{2}(N)$ so that $S \cong R$. Then $N=R P, S=R \cap G$, and $R \cong \mathbf{D}_{8}$. Therefore $S \cong \mathbf{D}_{8}$ or $\mathbf{Z}_{4}$ by $(2.1)-(7)$ because $P$ is not cyclic.

Let $q$ be the nondegenerate quadratic form on $V$ so that

$$
N=\left\{x \in G L(V) \mid q\left(v^{x}\right)=q(v) \text { for all } v \in V\right\} .
$$

We can take a basis $\left\{v_{1}, v_{2}, v_{3}, v_{4}\right\}$ of $V$ so that

$$
(\mathcal{Q}) \quad\left\{\begin{array}{l}
q\left(v_{i}\right)=1 \quad(1 \leqq i \leqq 4), \\
f\left(v_{i}, v_{j}\right)= \begin{cases}0 & (i+j \neq 5) \\
1 & (i+j=5)\end{cases}
\end{array}\right.
$$

where $f$ is the bilinear form on $V$ associated with $q$. Define the elements $\sigma, \tau, \varphi$, $\psi$ of $L$ by $(\mathcal{R})$. Then we have $\sigma, \tau, \varphi, \psi \in N$ by $(\mathcal{Q})$. Since $\sigma^{2}=\tau^{2}=(\sigma \tau)^{4}=1$ and $\varphi^{3}=\psi^{3}=[\varphi, \psi]=1$ by $(\mathcal{R}),\langle\sigma, \tau\rangle$ is a Sylow 2-subgroup of $N$ and $\langle\varphi, \psi\rangle=$ $O^{2}(N)=P$. Thus, replacing $R$ by its $N$-conjugate if necessary, we have

$$
R=\langle\sigma, \tau\rangle, S \supseteq\langle\sigma \tau\rangle, Z=\left\langle(\sigma \tau)^{2}\right\rangle .
$$

Note that $V_{n-1} \neq V_{n}$ if $V_{n-1} \neq 0$. Therefore we have

$$
(\mathcal{V})\left\{\begin{array}{l}
V_{1}=\left\langle v_{1}+v_{3}, v_{1}+v_{2}, v_{3}+v_{4}\right\rangle \\
V_{2}=\left\langle v_{1}+v_{4}, v_{2}+v_{3}\right\rangle \\
V_{3}=\left\langle v_{1}+v_{2}+v_{3}+v_{4}\right\rangle \\
V_{4}=0
\end{array}\right.
$$

by $(\mathcal{R})$, so $\left|V_{n-1}: V_{n}\right|=2(1 \leqq n \leqq 4)$. Thus

$$
V=V_{0} \supseteq V_{1} \supseteq V_{2} \supseteq V_{3} \supseteq V_{4}=0
$$

is the unique $S$-composition series of $V$, and, in particular, $V_{3}=C_{V}(S)$. Let $D=$ $\langle\sigma, Z\rangle$ and $C=\langle\tau, Z\rangle$. Then $\left[V_{2}, D\right]=0 \neq\left[V_{2}, C\right]$ by $(\mathcal{R})$ and $(\mathcal{V})$, so $D=C_{R}\left(V_{2}\right)$ and $E=S \cap D \neq S$. Thus $|S: E|=|R: D|=2$, and so $Z \subseteq E$. Moreover, $(\mathcal{R})$ and $(\mathcal{V})$ show that $V_{2}=[V, U]=C_{V}(U)$ for $U=Z$ or $D$, and that $[V, C, C]=V_{3}$. 
Therefore we conclude that $D$ is the unique quadratic fours subgroup of $R$. This proves (1)-(4).

Let $A$ be an elementary abelian subgroup of $S$.

To prove (5), suppose $\left|V: C_{V}(A)\right| \leqq|A|$ or $|[V, A]| \leqq|A|$. We may assume $A \neq 1$. If $A \subseteq C$, then $\left|V: C_{V}(A)\right|>|A|<|[V, A]|$ by $(\mathcal{R})$, a contradiction. Therefore we have $Z \nsupseteq A \subseteq D$, and hence $\left|V: C_{V}(A)\right|=|A|=|[V, A]|$ by $(\mathcal{R})$.

For the proof of (6) and (7), we may assume $Z \supsetneqq A \subseteq E$, and so $S \cong \mathbf{D}_{8}$ and $C_{S}(A)=E$. Hence we may also assume $B=E$ in proving (6). Computation using $(\mathcal{R})$ and $(\mathcal{V})$ shows that $V_{3} \nsupseteq[V, A] \subseteq V_{2} \subseteq C_{V_{1}}(A) \subset V_{1}$ and that $\left[C_{V}(A), E\right] \nsubseteq[V, A]$ if $A \neq E$. Thus

$$
V_{2}=V_{3}+[V, A]=C_{V_{1}}(A)=[V, A]+\left[C_{V}(A), E\right] .
$$

In proving (8), let $V_{2} \subset W \subset V$. Computation shows that $[V, A] \nsupseteq V_{3}$ if $Z \nsubseteq A$ and that $V_{3} \supsetneqq[W, D]=[W, Z] \subset V_{2}$ if $W \neq V_{1}$.

Suppose first that $[W, A] \supseteq V_{3}$. Then the above remark shows that $Z \cong A$, and then it also shows that $W=V_{1}$. Hence (i) and (ii) hold because $\left[V_{1}, D\right]=V_{2}$ and $\left[V_{1}, Z\right]=V_{3}$.

Suppose $[W, A]=0$. Then $W \neq V_{1}$ by $(7)$, so $[W, Z] \neq 0$ by the above. Hence we have $Z \nsubseteq A$, so (iii) holds.

Let $T \subseteq S$. If $T \subseteq E$, then (9) holds by (7), and (10) holds because $[V, T] \subseteq V_{2}$ also by (7). Computation shows that $C_{V}(\sigma \tau)=V_{3}=C_{V}(C)$ and that $[V, t]=C_{V}(t) \supseteqq V_{3}$ for $t \in C-Z$. Thus (9) and (10) also hold if $T \nsubseteq E$.

Suppose $S \cong \mathbf{D}_{8}$. Let $H=E P$, and put $K=\left\langle E, E^{x}\right\rangle$ for $x \in P$. Note that $H \cong \Sigma_{3} \times \Sigma_{3}$, and so $Z(H)=1$.

First, we will prove that $K=\langle E, x\rangle$, so we may assume $K \neq H$. Then $K=$ $E(K \cap P)=E^{x}(K \cap P) \cong \mathbf{D}_{12}$, so $1 \neq Z(K)=C_{E}(K \cap P)=C_{E^{x}}(K \cap P)=Z(K)^{x}$. Therefore $x \in C_{H}(Z(K))=K$, and hence $K=\langle E, x\rangle$.

Now, we prove (11). We may assume $x=y$ because $E^{x}=E^{y}$ and $V_{2}{ }^{x}=V_{2}{ }^{y}$. Let $1 \neq t \in E \cap E^{x}$. Then $K \subseteq C_{H}(t) \subset H$ and $0 \neq[V, t] \subseteq V_{2} \cap V_{2}{ }^{x}$. This shows that (i) implies (ii) and (iv). If $H \neq K$, then $\langle x\rangle=K \cap P \triangleleft K P=H$, and if $\langle x\rangle \triangleleft H$, then $1 \neq C_{E}(x) \subseteq E \cap E^{x}$. This shows that (ii) implies (iii) and that (iii) implies (i). Note that $\left[V_{2} \cap V_{2}{ }^{x}, K\right]=0$. Thus we have $H \neq K$ and $C_{V}(x) \neq 0$ if $V_{2} \cap V_{2}{ }^{x} \neq 0$. This shows that (iv) implies (ii) and (v). Suppose $C_{V}(x) \neq 0$. Then we have $C_{V_{2}}(x)=C_{C_{V}(x)}(Z) \neq 0$ because $C_{V}(Z)=V_{2}$ and $Z$ inverts $x$. Thus $V_{2} \cap V_{2}{ }^{x} \neq 0$. This shows that (v) implies (iv).

To prove (12), we may assume $x \in P$ as in (11). Let $\Delta$ be the set of subgroups of $P$ of order 3 , and put $\Delta_{1}=\{X \in \Delta \mid X \triangleleft H\}$ and $\Delta_{2}=\{X \in \Delta \mid X \not H\}$. Then $\left|\Delta_{1}\right|=\left|\Delta_{2}\right|=2$ by the structure of $H$, and $G$ leaves $\Delta_{1}$ and $\Delta_{2}$ invariant. Thus we have $\left|G: N_{G}(\langle x\rangle)\right| \leqq 2$, and so $C_{S}(x) \neq 1$ because $|S|=2^{3}$ and $\mid N_{G}(\langle x\rangle)$ : $C_{G}(x) \mid \leqq 2$. Since $G=\left\langle S, S^{x}\right\rangle$ and $O_{2}(G)=1$, we have $\left|S \cap S^{x}\right| \leqq 2$, so $S \cap S^{x}=$ 
$C_{S}(x)$. Consequently, if $C_{S}(x) \subseteq E$ then $C_{S}(x)=E \cap E^{x}=E \cap S^{x}=S \cap E^{x}$, and if $C_{S}(x) \varsubsetneqq E$ then $1=E \cap E^{x}=E \cap S^{x}=S \cap E^{x}$.

Lemma 2.6 Let $G \cong O_{4}^{+}(2)$, and let $V$ be an at most five dimensional $G F(2) G$-module. Suppose $(G, \bar{V}) \ll\left(O_{4}^{+}(2), G F(2)^{4}\right)$ for $\bar{V}=V / C_{V}(G)$. Denote by $\mathcal{J}$ the set of subgroups $J$ of $G$ of order 3 with $C_{\bar{V}}(J)=0$. Also, denote by $\mathcal{W}$ the set of $S$-invariant subspaces $W$ with $\bar{W}=C_{\bar{V}}(E)$ and $V=\left\langle W^{G}\right\rangle$, where $S$ ranges over the set $\operatorname{Syl}_{2}(G)$ and $E$ is the fours subgroup of $S$ acting quadratically on $V$. Then, for a given hyperplane $H$ of $V$, there exist $J \in \mathcal{J}$ and $W \in \mathcal{W}$ such that $H$ contains at most one member of $W^{J}=\left\{W^{x} \mid x \in J\right\}$.

Proof. Suppose false for some hyperplane $H$ of $V$. Let $W \in \mathcal{W}$. Then $0 \neq$ $C_{V}(G) \varsubsetneqq W$ because $\bar{V}=\bar{W}+\bar{W}^{x}$ for all $x \in O^{2}(G)$ with $C_{\bar{V}}(x)=0$ by (2.5)-(11). Moreover, replacing $W$ by its conjugate, if necessary, we may assume $W \subseteq H$.

Let $\left\{v_{0}, v_{1}, v_{2}, v_{3}, v_{4}\right\}$ be a basis of $V$ so that $C_{V}(G)=\left\langle v_{0}\right\rangle$, that $[V, G]=$ $\left\langle v_{1}, v_{2}, v_{3}, v_{4}\right\rangle$, and that the representation of $G$ on $[V, G]$ is given in (2.5)-(2). We will use the notation introduced there. Then

$$
S=\langle\sigma, \tau\rangle \quad \text { and } \quad E=\left\langle\sigma,(\sigma \tau)^{2}\right\rangle .
$$

Since $W \cap[V, G]=C_{[V, G]}(S)=\left\langle v_{1}+v_{2}+v_{3}+v_{4}\right\rangle$, we have

$$
W=\left\langle v_{0}+v_{1}+v_{4}, v_{1}+v_{2}+v_{3}+v_{4}\right\rangle
$$

and so

$$
\begin{aligned}
& W^{\varphi \psi}=\left\langle v_{0}+v_{1}, v_{1}+v_{2}\right\rangle, \quad W^{\varphi \psi^{-1}}=\left\langle v_{0}+v_{1}, v_{1}+v_{3}\right\rangle \\
& W^{\varphi^{-1} \psi}=\left\langle v_{0}+v_{4}, v_{2}+v_{4}\right\rangle, \quad W^{\varphi^{-1} \psi^{-1}}=\left\langle v_{0}+v_{4}, v_{3}+v_{4}\right\rangle
\end{aligned}
$$

by calculation using $(\mathcal{R})$ in (2.5)-(2). Our hypothesis forces that either $W^{\varphi \psi} \subseteq H$ or $W^{\varphi^{-1}} \psi^{-1} \subseteq H$, and that either $W^{\varphi^{-1} \psi} \subseteq H$ or $W^{\varphi \psi^{-1}} \subseteq H$. Then we have $H=V$ for all of the possible four cases, a contradiction.

Lemma 2.7 Let $G$ be a 2 -irreducible $\{2,3\}$-group with $O_{2}(G)=1, S \in \operatorname{Syl}_{2}(G)$, $P=O^{2}(G) \cong E s p_{27}$, and $V$ a six dimensional faithful $G F(2) G$-module. Put $\langle t\rangle=$ $\Omega_{1}(Z(S)), Z=\Omega_{1}(Z(P))$, and $T=C_{S}(Z)$. Then the following holds.

(1) $|S: T| \leqq 2, t \in T, C_{V}(Z)=0$, and $P$ is irreducible on $V$.

(2) $\quad\left|V / C_{V}(t)\right|=\left|C_{V}(t) /[V, t]\right|=|[V, t]|=2^{2}$.

(3) If $W=V / C_{V}(t), C_{V}(t) /[V, t]$, or $[V, t]$, then $C_{S Z}(W)=T$ and $(S Z, W)$ $\ll\left(G L_{2}(2), G F(2)^{2}\right)$.

(4) $S$ (resp. $T$ ) is embedded in $S D_{16}$ (resp. $\left.\mathbf{Q}_{8}\right),|T| \geqq 4$, all the elements of $S$ of order 4 are contained in $T$, and $[V, U]=C_{V}(t)$ and $\left[C_{V}(t), U\right]=[V, t]$ for all subgroups $U$ with $\langle t\rangle \subset U \subseteq T$. 
(5) If $S \neq T$, then $\left|V: C_{V}(S)\right|=\left|C_{V}(S)\right|=2,[V, S, t]=C_{V}(S)$, $|[V, S, S]|>2^{2},[V, S, S, S] \neq 0$, and $S \cong \mathbf{Z}_{8}, \mathbf{D}_{8}$, or $S D_{16}$.

(6) Let $A$ be an elementary abelian subgroup of $S$.

(ii) If $\left|V: C_{V}(A)\right| \leqq 2^{3}$, then $|A| \leqq 2$.

Proof. First, since $Z \cong \mathbf{Z}_{3}$, we have $|S: T| \leqq 2$. Also, (2.1)-(7) shows that $|S| \geqq 4$ because $P / Z \cong \mathbf{E}_{3^{2}}$. Thus $T \neq 1$, and so $t \in T$. Since $G L_{5}(2)$ has abelian Sylow 3-subgroups, $P$ is irreducible on $V$, and hence $C_{V}(Z)=0$.

Let $H=T P$ and $F=G F\left(2^{2}\right)$. Then $V$ is regarded as a three dimensional $F H$ module by Schur's lemma because $Z \subseteq Z(H)$. Thus $t$ induces an $F$-transvection on $V$, and so $\left|V / C_{V}(t)\right|=\left|C_{V}(t) /[V, t]\right|=|[V, t]|=2^{2}$. Let $W=V / C_{V}(t), C_{V}(t) /[V, t]$, or $[V, t]$. Then we have $(S Z, W) \ll\left(G L_{2}(2), G F(2)^{2}\right)$. Since $W$ is considered to be a one dimensional $F T$-module, we have $[W, T]=0$, and hence $C_{S Z}(W)=T$. This proves (1)-(3).

Let $\bar{P}=P / Z$. Then $S$ is embedded into $G L(\bar{P})$ because $S$ is faithful on $\bar{P}$. Thus $T=S \cap S p(\bar{P}) \subseteq S L(\bar{P})$. Therefore $S$ (resp. $T$ ) is isomorphic to a subgroup of $S D_{16}$ (resp. $\mathrm{Q}_{8}$ ), and hence all the elements of $S$ of order 4 are contained in $T$. Suppose $|T|=2$. Then $S \cong \mathbf{Z}_{4}$ by (1) and (2.1)-(7). Thus $S=T$ by the above, a contradiction. This shows that $|T| \geqq 4$. Let $\langle t\rangle \subset U \subseteq T$, and suppose either $[V, U] \subset C_{V}(t)$ or $\left[C_{V}(t), U\right] \subset[V, t]$. Since $V$ is an $F U$-module, we have $\operatorname{dim}_{F}([V, U])=1$ or $\operatorname{dim}_{F}\left(C_{V}(U)\right)=2$, and so $\left[V, U^{2}\right] \subseteq[V, U, U]=0$ in either case. Hence $U^{2}=1$, a contradiction. Thus $[V, U]=C_{V}(t)$ and $\left[C_{V}(t), U\right]=[V, t]$. This proves (4).

Suppose $S \neq T$. Pick $u \in S-T$, and let $W=[V, T]$ or $C_{V}(T)$. Then $\left|W: C_{W}(u)\right|=\left|C_{W}(u)\right|=2$ by (3). Therefore $[V, S]=[V, T]+[V, u]$ and $C_{V}(S)=C_{C_{V}(T)}(u)$, and then $|V:[V, S]|=\left|C_{V}(S)\right|=2$. Thus, as $[V, S, t]$ is $S$ invariant and of order $2,[V, S, t]=C_{V}(S)$. Since $[V, S, T] \supset C_{V}(T) \supset C_{V}(S)$, we also have $[V, S, S, S] \neq 0$ and $|[V, S, S]|>2^{2}$. Since $|S| \geqq 8$ and $S$ is not generated by elements of order 4 by (1) and (4), we conclude that $S \cong \mathbf{Z}_{8}, \mathbf{D}_{8}$, or $S D_{16}$, so (5) holds.

For the proof of (6), note that $A \cap T \subseteq\langle t\rangle$ by (4). If $[V, t, A]=0$, then $A \subseteq C_{S}([V, t])=T$ by (3), so (i) holds. Suppose $|A|>2$. Then $A \nsubseteq T$, and so $A$ contains an involution $u$ so that $A=\langle t, u\rangle$ and that $u$ inverts $Z$. Hence $\left|V: C_{V}(A)\right|=\left|V: C_{V}(t)\right| \cdot\left|C_{V}(t): C_{C_{V}(t)}(u)\right|=2^{2} \cdot 2^{2}>2^{3}$ by $(2)$ and $(1.2)$ because $C_{C_{V}(t)}(Z)=0$ by (1). This proves (ii).

Now, we determine the possibility of the pairs of 2-irreducible solvable groups and their $G F(2)$-representations under the hypothesis that some 2-subgroup has a high dimensional centralizer.

Lemma 2.8 Let $t$ be an involution acting on a p-group $P$ for some odd prime 
$p$ with $P=[P, t]$, and $V$ a faithful $G F(2) P\langle t\rangle$-module with $C_{V}(P)=0$. Suppose $\left|V: C_{V}(t)\right| \leqq 2^{3}$. Then one of the thirteen cases listed in the following table occurs.

\begin{tabular}{|c|c|c|c|c|c|}
\hline & $P\langle t\rangle, V)$ & $P$ & & $n(V)$ & $m\left(V / C_{V}(t)\right)$ \\
\hline (1) & 1 & $\mathbf{Z}_{3}$ & & 2 & 1 \\
\hline (2) & 1 & $\mathbf{Z}_{5}$ & & 4 & 2 \\
\hline (3) & 1 & $\mathbf{Z}_{7}$ & & 6 & 3 \\
\hline (4) & 1 & $\mathbf{Z}_{9}$ & & 6 & 3 \\
\hline (5) & 1 & $E s p_{27}$ & & 6 & 2 \\
\hline (6) & 1 & $\mathbf{Z}_{3} \mathrm{wr}_{\mathbf{Z}} \mathbf{Z}_{3}$ & & 6 & 3 \\
\hline (7) & 2 & $\mathbf{Z}_{3}$ & & 4 & 2 \\
\hline (8) & 2 & $\mathbf{E}_{3^{2}}$ & & 4 & 2 \\
\hline (9) & 2 & $E s p_{27}$ & & 8 & 3 \\
\hline$(10)$ & 2 & $Z_{3} \times E s p_{2}$ & & 8 & 3 \\
\hline & $n(P\langle t\rangle, V)$ & $P$ & $m(V)$ & \multicolumn{2}{|c|}{$m\left(V / C_{V}(t)\right)$} \\
\hline (11) & 3 & $\mathbf{Z}_{3}$ & 6 & \multicolumn{2}{|r|}{3} \\
\hline$(12)$ & 3 & $\mathbf{E}_{3^{2}}$ & 6 & \multicolumn{2}{|r|}{3} \\
\hline (13) & 3 & $\mathbf{E}_{3^{3}}$ & 6 & \multicolumn{2}{|r|}{3} \\
\hline
\end{tabular}

Moreover, $C_{V}(\Phi(P)) \neq 0$ in the cases (9) and (10).

Proof. First, we deal with the case where $P\langle t\rangle$ is irreducible on $V$. Put $Z=Z(P)$.

Assume first that $[Z, t] \neq 1$. Put $Z_{0}=[Z, t]$. Since $C_{V}\left(Z_{0}\right)=0$ and $t$ inverts $Z_{0}$, we have $|V|=\left|V: C_{V}(t)\right|^{2}=2^{2 n}(n \leqq 3)$ by (1.2). Let $W$ be an irreducible $G F(2) Z_{0}$-submodule of $V$. Then $Z_{0} / C_{Z_{0}}(W)$ is cyclic by Schur's lemma. Since $C_{V}\left(C_{Z_{0}}(W)\right)$ is $P$-invariant, we have $C_{V}\left(C_{Z_{0}}(W)\right)=V$, so $C_{Z_{0}}(W)=1$. Hence $Z_{0}$ is cyclic.

Suppose $P$ is abelian. Then $Z_{0}=P$, and so, inspecting irreducible dihedral subgroups of $G L_{2 n}(2)$, we have $P \cong \mathbf{Z}_{3}$ if $n=1, \mathbf{Z}_{5}$ if $n=2, \mathbf{Z}_{7}$ or $\mathbf{Z}_{9}$ if $n=3$.

Suppose $P$ is nonabelian. Then, inspecting nonabelian $p$-subgroups of $G L_{6}(2)$, we have $|V|=2^{6}$ and $p=3$. Suppose $P$ is an extraspecial group of order 27 , and let $\bar{P}=P / Z$. Since $t$ inverts $\bar{P}$, we have $t \in S L(\bar{P})=S p(\bar{P})$, and so $[Z, t]=1$, a contradiction. Thus we have $P \cong \mathbf{Z}_{3}{ }_{\text {wr }} \mathbf{Z}_{3}$ (the wreath product of $\mathbf{Z}_{3}$ by $\mathbf{Z}_{3}$ ).

Assume $[Z, t]=1$. Then $P$ is nonabelian, and, moreover, $Z$ is cyclic by Schur's lemma because $Z \cong Z(P\langle t\rangle)$. Let $Z \subseteq L \subseteq P$ so that $L \triangleleft P\langle t\rangle$ and $|L: Z|=p$, and put $K=\Omega_{1}(L)$. Since $P \neq C_{P}(L), L$ is noncyclic abelian, so $L=K Z$ and $K \cong \mathbf{E}_{p^{2}}$. Thus $C_{P}(K)=C_{P}(L) \neq P=[P, t]$, and hence $[K, t] \neq 1$. Let $\Delta$ be the set of subgroups of $K$ of order $p$ distinct from $K \cap Z$. Then $V=\bigoplus_{X \in \Delta} C_{V}(X)$ by $(2.1)$ of $[\mathbf{1}]$ because $C_{V}(K \cap Z)=0$. Since $|\Delta|=p$ and $P$ acts transitively on 
$\Delta,|V|=\left|C_{V}(X)\right|^{p}$, and so $|[V, X]|=\left|V: C_{V}(X)\right|=\left|C_{V}(X)\right|^{p-1}$ for all $X \in \Delta$. Now, put $X=[K, t]$. Since $t$ inverts $X$ and $X \in \Delta,|[V, X]|=\left|[V, X]: C_{[V, X]}(t)\right|^{2}$ $\leqq\left|V: C_{V}(t)\right|^{2} \leqq 2^{6}$, and so $\left|C_{V}(X)\right|^{p-1} \leqq 2^{6}$. Note that $\left|C_{V}(X)\right| \geqq 2^{2}$ because $C_{C_{V}(X)}(Z)=0$. Therefore we have $p=3$ and $\left|C_{V}(X)\right|=2^{2}$, and so $|V|=2^{6}$. Hence $P\langle t\rangle$ is embedded in $G L_{3}\left(2^{2}\right)$ by Schur's lemma because $Z \cong Z(P\langle t\rangle)$. Thus $P=[P, t]$ is embedded in $G L_{3}\left(2^{2}\right)^{\prime}=S L_{3}\left(2^{2}\right)$, and so $P \cong E s p_{27}$.

Now, let $\left\{W_{1}, \ldots, W_{n}\right\}(n>1)$ be the set of $P\langle t\rangle$-composition factors of $V$. Then $\prod_{i=1}^{n}\left|W_{i}: C_{W_{i}}(t)\right| \leqq\left|V: C_{V}(t)\right| \leqq 2^{3}$ by (1.3), so $n \leqq 3$ and we may assume $\left|W_{1}: C_{W_{1}}(t)\right|=2$. Therefore we have $P / C_{P}\left(W_{1}\right) \cong \mathrm{Z}_{3}$ and $\left|W_{1}\right|=2^{2}$ by a result for the irreducible case, and hence $P$ is a 3-group.

Suppose $n=2$. Then, since $\left|W_{2}: C_{W_{2}}(t)\right| \leqq 2^{2}, P / C_{P}\left(W_{2}\right) \cong \mathbf{Z}_{3}$ and $\left|W_{2}\right|=$ $2^{2}$, or $P / C_{P}\left(W_{2}\right) \cong E s p_{27}$ and $\left|W_{2}\right|=2^{6}$. Therefore we have $P \cong \mathbf{Z}_{3}$ or $\mathbf{E}_{3^{2}}$ and $|V|=2^{4}$, or $P \cong E s p_{27}$ or $\mathbf{Z}_{3} \times E s p_{27}$ and $|V|=2^{8}$.

Suppose $n=3$. Then, since $\left|W_{2}: C_{W_{2}}(t)\right|=\left|W_{3}: C_{W_{3}}(t)\right|=2, P / C_{P}\left(W_{2}\right) \cong$ $P / C_{P}\left(W_{3}\right) \cong \mathbf{Z}_{3}$ and $\left|W_{2}\right|=\left|W_{3}\right|=2^{2}$. Therefore we have $P \cong \mathbf{Z}_{3}, \mathbf{E}_{3^{2}}$, or $\mathbf{E}_{3^{3}}$ and $|V|=2^{6}$.

Finally, we determine the index $\left|V: C_{V}(t)\right|$. If $P$ is abelian, then $\left|V: C_{V}(t)\right|=$ $|V|^{1 / 2}$ by (1.2). If $P$ is nonabelian, then $n=2$ and $1 \neq \Phi(P) \subseteq C_{P}\left(W_{1}\right)$, and so $V=C_{V}(\Phi(P)) \oplus[V, \Phi(P)] \cong W_{1} \oplus W_{2}$. Therefore $\left|V: C_{V}(t)\right|=\left|W_{1}: C_{W_{1}}(t)\right|$. $\left|W_{2}: C_{W_{2}}(t)\right|$.

Now, for a group $G$, define

$$
n_{*}(G)=\max \left\{n_{p}(G) \mid 2 \neq p \in \pi(G)\right\} .
$$

Suppose $O_{2}(G) \neq 1$. Then $G$ is itself a 2 -local subgroup of $G$, and hence

$$
n_{*}(G) \leqq f(G) \text {. }
$$

Suppose, moreover, that $G$ is 2-irreducible and solvable. Then (2.1)-(3) shows that $\pi(G)=\{2, p\}$ for some odd prime $p$, and then we have $n_{*}(G)=n_{p}(G)$. Therefore $G$ is thin if and only if $n_{*}(G)=1$ by $(2.1)-(7)$.

Proposition 2.9 Let $G$ be a 2-irreducible solvable group, $S$ a Sylow 2-subgroup of $G, V$ an irreducible faithful $G F(2) G$-module, and $t$ an involution of $S$.

(1) If $t \in Z(S)$ and $\left|V: C_{V}(t)\right| \leqq 2^{3}$, then one of the six cases listed in the following table occurs.

$\begin{array}{cccc} & O^{2}(G) & m(V) & m\left(V / C_{V}(t)\right) \\ (1-1) & \mathbf{Z}_{3} & 2 & 1 \\ (1-2) & \mathbf{Z}_{5} & 4 & 2 \\ (1-3) & \mathbf{Z}_{7} & 6 & 3 \\ (1-4) & \mathbf{Z}_{9} & 6 & 3 \\ (1-5) & E s p_{27} & 6 & 2 \\ (1-6) & \mathbf{E}_{3^{2}} & 4 & 2\end{array}$


(2) If $t \notin Z(S),\left|V: C_{V}(t)\right| \leqq 2^{2}$, and $n_{*}(G) \leqq 2$, then one of the three cases listed in the following table occurs.

$\begin{array}{cccc} & O^{2}(G) & m(V) & m\left(V / C_{V}(t)\right) \\ (2-1) & \mathbf{E}_{3^{2}} & 4 & 1 \\ (2-2) & \mathbf{E}_{3^{2}} & 4 & 2 \\ (2-3) & \mathbf{E}_{5^{2}} & 8 & 2\end{array}$

Proof. Let $P=O^{2}(G)$. Since $P \neq 1$ by $(2.1)-(1)$ and $G$ is irreducible and faithful on $V, C_{V}(P)=0$ and $O_{2}(G)=1$, and then $P$ is a Sylow $p$-subgroup of $G$ for some odd prime $p$ by $(2.1)-(3)$.

Suppose first that $t \in Z(S)$ and $\left|V: C_{V}(t)\right| \leqq 2^{3}$. Then $P=[P, t]$ by $(2.1)$ (5), and hence $P$ and $V$ are described as in (2.8). We have $P \neq \mathbf{Z}_{3}$ wr $\mathbf{Z}_{3}$ by the 2-irreducibility of $G$, and $m(V) \neq 8$ because $G$ is irreducible on $V$. If $P \cong \mathbf{Z}_{3}$, then $G \cong \Sigma_{3}$ and $|V|=2^{2}$ also because $G$ is irreducible on $V$. Suppose $P \cong \mathbf{E}_{3^{2}}$ or $\mathbf{E}_{3^{3}}$, and regard $V$ as a $G F(2) P$-module. Then Clifford's theorem shows that $V=W_{1} \oplus \cdots \oplus W_{s}$ where $W_{1}, \ldots, W_{s}$ are the homogenous components of $V$. Since $C_{V}(P)=0$, we have $\left|W_{i}\right| \geqq 2^{2}(1 \leqq i \leqq s)$, so $2^{2 s} \leqq|V| \leqq 2^{6}$ by (1.2). This shows that $|V|=2^{4}$ because $s>1$ and $s$ is a power of 2 , and hence $P \cong \mathbf{E}_{3^{2}}$.

Suppose next that $t \notin Z(S)$, that $\left|V: C_{V}(t)\right| \leqq 2^{2}$, and that $n_{p}(G)=$ $n_{*}(G) \leqq 2$. Then $|P / \Phi(P)|=p^{2}$ and $m(S)=2$ by $(2.1)-(6)$. We can take $u \in t^{S}$ such that $\Omega_{1}(Z(S)) \subseteq\langle t, u\rangle \cong \mathbf{E}_{2^{2}}$ by (1.1). Define

$$
P_{t}=[P, t], P_{u}=[P, u], V_{t}=\left[V, P_{t}\right], \quad \text { and } \quad V_{u}=\left[V, P_{u}\right] .
$$

Then $P_{t}$ and $V_{t}$ are described as in (2.8), and, moreover, $P=\left[P, \Omega_{1}(Z(S))\right]=P_{t} P_{u}$, and so $V=[V, P]=V_{t}+V_{u}$. Let $\bar{P}=P /\left(P_{t} \cap P_{u}\right)$. Then $\bar{P}_{t}$ is cyclic because $|P / \Phi(P)|=p^{2}$ and $\bar{P}_{t} \cong \bar{P}_{u}$. Thus, noting that $\left(P_{t}\right)^{p}=1$ for all of the possible cases listed in (2.8), we have $\left|\bar{P}_{t}\right|=p$, and so $P_{t} \cap P_{u}=\Phi(P)$.

Suppose $P_{t} \cong \mathbf{E}_{3^{2}}$. Then $P_{t} \cong E s p_{27}$ and $t$ inverts $Z(P)=\Phi(P)$. Therefore $|V|=\left|V: C_{V}(t)\right|^{2} \leqq 2^{4}$ by $(1.2)$ as $C_{V}(Z(P))=0$, which is a contradiction because $G L_{4}(2)$ has abelian Sylow 3 -subgroups. Suppose $P_{t} \cong E s p_{27}$. Then, as $Z(P) \neq \Phi(P)$ and $|\Phi(P)|=9, \Phi(P) \subset C_{P}(\Phi(P)) \subset P$, which contradicts the 2-irreducibility of $G$. Therefore, we have $P_{t} \cong P_{u} \cong \mathbf{Z}_{3}$ or $\mathbf{Z}_{5},\left|V_{t}\right|=\left|V_{u}\right|=2^{2}$ or $2^{4}$, and $P=P_{t} \times P_{u}$. Since $|V| \leqq\left|V_{t}\right| \cdot\left|V_{u}\right|$ and $P$ is faithful on $V$, we have $|V|=2^{8}$ if $P \cong \mathbf{E}_{5^{2}}$, and $2^{4} \leqq|V| \leqq 2^{8}$ if $P \cong \mathbf{E}_{3^{2}}$.

Suppose $P \cong \mathbf{E}_{3^{2}}$. Then $P_{t}\langle t\rangle \cong P_{u}\langle u\rangle \cong \Sigma_{3}$, and it follows from the argument in the second paragraph that $|V|=2^{4}$ or $2^{8}$. Assume $|V|=2^{8}$. Then we have $V=V_{t} \oplus V_{u}$, so $C_{P}\left(V_{t}\right)=P_{u}$ and $C_{P}\left(V_{u}\right)=P_{t}$. Therefore, regarded as a $G F(2) P$ module, $V_{t}$ and $V_{u}$ are the homogenous components of $V$, and hence $G$ permutes $P_{t}$ and $P_{u}$. Thus $\left|G: P_{t}\langle t\rangle \times P_{u}\langle u\rangle\right|=2$ because Aut $\left(\Sigma_{3}\right) \cong \Sigma_{3}$ and $O_{2}(G)=1$. Now, let $W$ be an irreducible $G F(2)\left(P_{t}\langle t\rangle \times P_{u}\langle u\rangle\right)$-submodule of $V_{t}$. Then $|W|=2^{2}$ as 
$\left[V_{t}, P_{u}\langle u\rangle\right]=0$, so $\left|\left\langle W^{G}\right\rangle\right| \leqq|W|^{2}=\left(2^{2}\right)^{2}<|V|$, which contradicts the fact that $G$ is irreducible on $V$. Thus we have $|V|=2^{4}$.

Lemma 2.10 Let $G$ be a 2-irreducible solvable group with $O_{2}(G)=1, S$ a Sylow 2-subgroup of $G$, and $V$ a faithful $G F(2) G$-module. Put $W=\left[V, O^{2}(G)\right]$, and let $A$ be an elementary abelian subgroup of $S$.

(1) Assume $\left|V: C_{V}(A)\right| \leqq|A| \neq 1$ and that $n_{*}(G) \leqq 2$. Then $W$ is irreducible, and

$$
\begin{aligned}
& O^{2}(G) \cong \mathbf{Z}_{3} \quad \text { and } \quad|W|=2^{2} \quad \text { if } \quad A \cong \Omega_{1}(Z(S)), \quad \text { and } \\
& O^{2}(G) \cong \mathbf{E}_{3^{2}} \quad \text { and } \quad|W|=2^{4} \quad \text { if } \quad A \nsubseteq \Omega_{1}(Z(S)) .
\end{aligned}
$$

Moreover, $\left|V: C_{V}(A)\right|=|A|$.

(2) Assume $\Omega_{1}(Z(S)) \subset A$, and that one of the following holds:

(i) $\left|V: C_{V}(A)\right| \leqq 2^{3}$;

(ii) $|[V, A]| \leqq 2^{3}$;

(iii) there exists an $A$-invariant subspace $U$ of $V$ such that $|V: U| \cdot|[U, A]| \leqq 2^{2}$.

Then $W$ is irreducible, and

$$
O^{2}(G) \cong \mathbf{E}_{3^{2}} \text { and }|W|=2^{4} .
$$

Moreover, $\left|V: C_{V}(A)\right|>2<|[V, A]|$.

Proof. Let $P=O^{2}(G)$. Throughout this proof, assume that the hypothesis of (1) or (2) is satisfied for the triple $(G, V, A)$.

First, we will assume that $V$ is irreducible.

Suppose $\Omega_{1}(Z(S)) \not \subset A$. Then we have $\left|V: C_{V}(A)\right| \leqq|A| \neq 1$, and, in particular, $|A|=2$ by (2.1)-(6). Thus (2.9) shows that

$$
\begin{aligned}
& P \cong \mathbf{Z}_{3} \text { and }|V|=2^{2} \text { if } A \cong \Omega_{1}(Z(S)), \text { and } \\
& P \cong \mathbf{E}_{3^{2}} \text { and }|V|=2^{4} \text { if } A \nsubseteq \Omega_{1}(Z(S)) .
\end{aligned}
$$

Therefore we have $\left|V: C_{V}(A)\right|=|A|$ by (2.5)-(4).

Suppose $\Omega_{1}(Z(S)) \subset A$. Then we may assume that the hypothesis of (2) is satisfied. Since $m(S) \geqq 2, P$ is not cyclic by $(2.1)-(7)$.

Suppose first that the condition (i) holds. Let $1 \neq t \in \Omega_{1}(Z(S))$. Then we have $\left|V: C_{V}(t)\right| \leqq 2^{3}$, and hence either

$$
P \cong \mathbf{E}_{3^{2}} \text { and }|V|=2^{4}, \quad \text { or } \quad P \cong E s p_{27} \text { and }|V|=2^{6}
$$

by (2.9). If $P \cong E s p_{27}$ and $|V|=2^{6}$, then $|A| \leqq 2$ by (2.7)-(6), a contradiction. Thus we have $P \cong \mathbf{E}_{3} 2$ and $|V|=2^{4}$, and hence $\left|V: C_{V}(A)\right|>2<|[V, A]|$ by $(2.5)-(5)$. 
Suppose next that the condition (ii) holds. Then the condition (i) holds for the dual module $V^{*}$ of $V$, and the conclusion is valid for $V^{*}$. Thus the conclusion also holds for $V$ because $|V|=\left|V^{*}\right|,\left|V: C_{V}(A)\right|=\left|\left[V^{*}, A\right]\right|$, and $|[V, A]|=$ $\left|V^{*}: C_{V^{*}}(A)\right|$.

Finally, suppose that the condition (iii) holds. We may assume that $|V: U|=$ $|[U, A]|=2$. Pick $s \in A-\{t\}$, and put $B=\langle t, s\rangle$. Then, as both $t$ and $s$ induce transvections on $U,\left|U: C_{U}(t)\right| \leqq 2 \geqq\left|U: C_{U}(s)\right|$, so $\left|U: C_{U}(B)\right| \leqq 2^{2}$. Thus we have $\left|V: C_{V}(B)\right| \leqq 2^{3}$. Hence the condition (i) holds for $B$, so $P \cong \mathbf{E}_{3^{2}}$ and $|V|=2^{4}$ and $\left|V: C_{V}(A)\right| \geqq\left|V: C_{V}(B)\right|>2<|[V, B]| \leqq|[V, A]|$.

We have shown that $(2.10)$ holds if $V$ is irreducible. Since $W$ is faithful by (2.4)-(2), it suffices to prove that $W$ is irreducible. This is done after the proof of (2.11).

Throughout the remainder of this paper, we will use the following terminology and notation.

Let $G$ be a group, $V$ a $G F(2) G$-module, and $A$ a subgroup of $G$. We will say that $A$ induces a transvection (resp. a quasitransvection) on $V$ if $\left|V: C_{V}(A)\right| \leqq 2$ (resp. $\left|V: C_{V}(A)\right| \leqq 2^{2}$ ). Put $\bar{G}=G / C_{G}(V)$. We will say that $A$ induces an offending action on $V$ if $\left|V: C_{V}(\bar{A})\right| \leqq|\bar{A}|$. Moreover, we will say that $A$ induces a semioffending action on $V$ if either $A$ induces an offending action on $V$, or the triple $(\bar{G}, V, \bar{A})$ satisfies the hypothesis of $(2.10)-(2)$.

Next, let $G$ be a group acting on a 2 -group $T$. We will denote by $n(G, T)$ the number of the nontrivial $G$-composition factors of $T$. A subgroup $A$ of $G$ induces a factorwise transvection, a factorwise quasitransvection, a factorwise offending action, or a factorwise semioffending action on $T$ if $A$ induces a transvection, a quasitransvection, an offending action, or a semioffending action, respectively, on each nontrivial $G$-composition factor of $T$ regarded as a $G F(2) G$-module. Also, $A$ induces a factorwise sesquitransvection on $T$ if $A$ induces a factorwise quasitransvection on $T$ and a transvection on at least one nontrivial $G$-composition factor of $T$. Let $H$ be another group. We will say that $T$ is induced by a $G F(2) H$-module $W$ and denote by $(G, T) \ll(H, W)$ if each $G$-composition factor of $T$ is either trivial or induced by $W$.

Lemma 2.11 Let $G$ be a 2-irreducible solvable group, and $S$ a Sylow 2-subgroup of $G$. Suppose $G$ acts on a 2-group $T$ with $\left[T, O^{2}(G)\right] \neq 1$. Put $Q=O_{2}(G)$, and take a subgroup $A$ of $S$ so that $A Q / Q$ is elementary abelian.

(1) Assume $A Q / Q \neq 1$ and that one of the following holds:

(i) A induces a factorwise quasitransvection on $T, G$ is a $\{2,3\}$-group, and $G$ has cyclic Sylow 3 -subgroups if $A Q / Q \subseteq \Omega_{1}(Z(S / Q))$;

(ii) $\quad A$ induces a factorwise sesquitransvection on $T$;

(iii) $A$ induces a factorwise semioffending action on $T$. 
Then we have

$$
\begin{aligned}
& C_{G}(W)=Q C_{G}(T), \\
& O^{2}\left(G / C_{G}(W)\right) \cong \mathbf{Z}_{3} \text { and }|W|=2^{2} \quad \text { if } \quad A Q / Q \cong \Omega_{1}(Z(S / Q)), \\
& O^{2}\left(G / C_{G}(W)\right) \cong \mathbf{E}_{3^{2}} \quad \text { and } \quad|W|=2^{4} \quad \text { if } \quad A Q / Q \nsubseteq \Omega_{1}(Z(S / Q)),
\end{aligned}
$$

where $W$ is an arbitrary nontrivial $G$-composition factor of $T$. Moreover, $|A Q / Q|=$ 2 if (ii) holds.

(2) Assume $|T: U| \cdot|[U, A]| \leqq 2^{\text {s }}$ for some $s \geqq 0$ and some $A$-invariant subgroup $U$ of $T$. Then $n(G, T) \leqq s$ if $A \nsubseteq Q$. Moreover, $|A Q / Q| \leqq 2$ and $A$ induces a factorwise sesquitransvection on $T$ if $s \leqq n^{\prime}$, where

$$
n^{\prime}=\left\{\begin{array}{lll}
n(G, T)+1 & \text { if } & n(G, T)>1 \\
1 & \text { if } & n(G, T)=1
\end{array}\right.
$$

(3) Assume $\Omega_{1}(Z(S / Q)) \subset A Q / Q$ and that one of the following holds:

(i) $\left|T: C_{T}(A)\right| \leqq 2^{2 s+1}$ for some $s \geqq 0$;

(ii) $|T: U| \cdot|[U, A]| \leqq 2^{2 s}$ for some $s \geqq 0$ and some $A$-invariant subgroup $U$ of $T$.

Then $n(G, T) \leqq s$. Moreover, $A$ induces a factorwise semioffending action on $T$ if $n(G, T)=s$.

Proof. By (2.1)-(3), we have $G=O_{2, p, 2}(G)$ for some odd prime $p$. Let $P$ be a Sylow $p$-subgroup of $G$, and let $W$ be an arbitrary nontrivial $G$-composition factor of $T$. Put $\bar{G}=G / C_{G}(W)$, and regard $W$ as an irreducible faithful $G F(2) \bar{G}$ module. Since $Q \subseteq C_{G}(W) \nsupseteq O^{2}(G)$, we have $C_{G}(W) \subseteq \Phi(P) Q,|\bar{A}|=|A Q / Q|$, and $\bar{A} \subseteq \Omega_{1}(Z(\bar{S}))$ if and only if $A Q / Q \subseteq \Omega_{1}(Z(S / Q))$ by (2.4)-(1). We remark, moreover, that $(2.10)$ holds for the triple $(\bar{G}, W, \bar{A})$.

To prove (1), assume first that $|A Q / Q|=2$. The definitions of a factorwise semioffending action and a factorwise sesquitransvection show that (iii) implies (ii). Suppose that (ii) holds, and choose $W$ on which $A$ induces a transvection. Then we have

$$
(*) \quad\left\{\begin{array}{l}
\bar{P} \cong \mathbf{Z}_{3} \text { and }|W|=2^{2} \text { if } A Q / Q \cong \Omega_{1}(Z(S / Q)), \\
\bar{P} \cong \mathbf{E}_{3^{2}} \text { and }|W|=2^{4} \text { if } A Q / Q \nsubseteq \Omega_{1}(Z(S / Q)) .
\end{array}\right.
$$

by (2.9). Therefore $G$ is a $\{2,3\}$-group in either case, and $P$ is cyclic if $A Q / Q \subseteq$ $\Omega_{1}(Z(S / Q)$ ), so (i) holds. Now, suppose (i) holds, and let $W$ be arbitrary. Then (2.9) also yields $(*)$. Thus $C_{G}(W)=\Phi(P) Q$ because $\bar{P}$ is elementary abelian, and hence $[T, \Phi(P)]=1$ because $W$ is arbitrary. This shows that $C_{G}(W)=Q C_{G}(T)$.

Assume next that $|A Q / Q|=2^{2}$. The definitions of a factorwise quasitransvection and a factorwise semioffending action show that (i) implies (iii). The condition (ii) never holds because $A$ does not induce a transvection on $W$ by (2.10)-(1). Now, 
suppose (iii) holds. Since $A Q / Q \nsubseteq \Omega_{1}(Z(S / Q))$ by $(2.1)-(4)$, we have $\bar{P} \cong \mathbf{E}_{3^{2}}$ and $|W|=2^{4}$ by $(2.10)-(2)$. Therefore, similarly as in the case where $|A Q / Q|=2$, we have $C_{G}(W)=Q C_{G}(T)$. This proves (1).

For the proof of (2) and (3), let

$$
T=T_{0} \supseteqq T_{1} \supseteqq \cdots \supseteqq T_{r-1} \supseteqq T_{r}=1
$$

be a $G$-composition series of $T$, and let $U$ be an $A$-invariant subgroup of $T$. Put $U_{i}=\left(T_{i-1} \cap U\right) T_{i}(1 \leqq i \leqq r)$. Suppose $W_{1}=T_{i_{1}-1} / T_{i_{1}}, \ldots, W_{n}=T_{i_{n}-1} / T_{i_{n}}$ $(n=n(G, T))$ are the nontrivial $G$-composition factors, and set $V_{k}=U_{i_{k}} / T_{i_{k}}$ $(1 \leqq k \leqq n)$. Then (1.3) shows that

$$
\begin{aligned}
|T: U| \cdot|[U, A]| & \geqq \prod_{i=1}^{r}\left|T_{i-1} / T_{i}: U_{i} / T_{i}\right| \cdot\left|\left[U_{i} / T_{i}, A\right]\right| \\
& \geqq \prod_{k=1}^{n}\left|W_{k}: V_{k}\right| \cdot\left|\left[V_{k}, A\right]\right| .
\end{aligned}
$$

Let $|T: U| \cdot|[U, A]| \leqq 2^{s}$.

Suppose $A \nsubseteq Q$. Since $A$ centralizes none of the nontrivial composition factor of $T$, we have $\left|W_{k}: V_{k}\right| \cdot\left|\left[V_{k}, A\right]\right| \geqq 2$ for all $k(1 \leqq k \leqq n)$, so

$$
2^{n} \leqq \prod_{k=1}^{n}\left|W_{k}: V_{k}\right| \cdot\left|\left[V_{k}, A\right]\right| \leqq|T: U| \cdot|[U, A]| \leqq 2^{s} .
$$

This shows that $n \leqq s$. Suppose $s \leqq n^{\prime}$. Then we have

$$
\begin{aligned}
& \left|W_{k}: V_{k}\right| \cdot\left|\left[V_{k}, A\right]\right| \leqq 2^{2} \quad \text { for all } \quad k(1 \leqq k \leqq n), \quad \text { and } \\
& \left|W_{m}: V_{m}\right| \cdot\left|\left[V_{m}, A\right]\right| \leqq 2 \text { for at least one } m(1 \leqq m \leqq n) .
\end{aligned}
$$

Hence $A$ induces a transvection on this $W_{m}$ or its dual module $W_{m}{ }^{*}$, and so $|A Q / Q|=2$ by (2.10)-(1). Therefore $A$ induces a quasitransvection on each $W_{k}$, and a transvection on $W_{m}$. Thus (2) holds.

Let $\Omega_{1}(Z(S / Q)) \subset A Q / Q$.

Suppose (i) of (3) holds. Then, letting $U=C_{T}(A)$ in (*), we have

$$
\prod_{k=1}^{n}\left|W_{k}: C_{W_{k}}(A)\right| \leqq\left|T: C_{T}(A)\right|=2^{2 s+1} .
$$

Therefore $n \leqq s$, and, moreover, $\left|W_{k}: C_{W_{k}}(A)\right| \leqq 2^{3}$ for all $k(1 \leqq k \leqq n)$ if $n=s$, because $\left|W_{k}: C_{W_{k}}(A)\right| \geqq 2^{2}$ for all $k(1 \leqq k \leqq n)$ by $(2.10)-(2)$. 
Suppose (ii) of (3) holds. Then, by (*), we also have $n \leqq s$ and $\left|W_{k}: V_{k}\right|$. $\left|\left[V_{k}, A\right]\right|=2^{2}$ for all $k(1 \leqq k \leqq n)$ if $n=s$, because $\left|W_{k}: V_{k}\right| \cdot\left|\left[V_{k}, A\right]\right| \geqq 2^{2}$ for all $k(1 \leqq k \leqq n)$ by $(2.10)-(2)$. Thus (3) holds.

Now, we return to the proof of $(2.10)$.

As stated before, it suffices to prove that $W=\left[V, O^{2}(G)\right]$ is irreducible under the hypothesis of (2.10).

Suppose the hypothesis of $(1)$ or $(2)$ of $(2.10)$ holds. Then there exists a unique nontrivial composition factor within $V$ by $(2.11)-(2)(3)$. Thus $W$ is indeed irreducible because $W=\left[W, O^{2}(G)\right]$ and $C_{W}\left(O^{2}(G)\right)=0$ by $(2.4)-(3)$. This completes the proof of $(2.10)$.

COROLlary 2.12 Let $G$ be a 2 -irreducible solvable group of even order with $\mathrm{O}_{2}(G)=1, S$ a Sylow 2-subgroup of $G$, and $V$ a faithful $G F(2) G$-module. Put $W=\left[V, O^{2}(G)\right]$.

(1) Suppose $|[V, S, S]| \leqq 2^{2}$. Then one of the following holds.

(i) $\quad G$ is dihedral and $[V, S, S]=0$.

(ii) $O^{2}(G) \cong \mathbf{Z}_{5},|W|=2^{4}$, and $|[W, S, S]|=2^{2}$.

(iii) $O^{2}(G) \cong \mathbf{E}_{3^{2}},|W|=2^{4}$, and $|[W, S, S]|=2^{2}$.

(iv) $\quad O^{2}(G) \cong E s p_{27},|W|=2^{6},|[W, S, S]|=2^{2}$, and $m(S)=1$.

Moreover, if $[V, S, S] \neq 0=[V, S, S, S]$ then $O^{2}(G) \cong E s p_{27}$.

Suppose $\left|V: C_{V}(S)\right| \leqq 2^{2}$. Then

$$
G \cong \mathbf{D}_{6} \quad \text { or } \quad \mathbf{D}_{10}, \quad \text { and } \quad|W|=2^{2} \quad \text { or } \quad 2^{4} .
$$

Proof. Let $P=O^{2}(G)$. Then $P$ is a Sylow $p$-subgroup for some odd prime $p$ by $(2.1)-(3)$.

Suppose $|[W, S, S]| \leqq|[V, S, S]| \leqq 2^{2}$. If $S^{2}=1$, then $|S|=2$ and $P$ is cyclic by (2.1)-(7), so (i) holds. Assume $S^{2} \neq 1$, and let $t \in \Omega_{1}(Z(S)) \cap S^{2}$. Then $t$ induces a quasitransvection on $W$ because $[W, t] \subseteq\left[W, S^{2}\right] \subseteq[W, S, S]$. Since $C_{W}(P)=0$ and $C_{G}(W)=1$ by $(2.4)-(2)(3)$, we have $P \cong \mathbf{Z}_{3}$ and $|W|=2^{2}$ or $2^{4}, P \cong \mathbf{Z}_{5}$ and $|W|=2^{4}, P \cong E s p_{27}$ and $|W|=2^{6}$, or $P \cong \mathbf{E}_{3^{2}}$ and $|W|=2^{4}$ by (2.8). Since $S^{2} \neq 1$, we have $P \neq \mathbf{Z}_{3}$, and then $t$ does not induce a transvection on $W$. Hence $|[W, t]|=|[W, S, S]|=2^{2}$. If $P \cong E s p_{27}$, then, by $(2.7)-(4)(5)$, we have $m(S)=1$. The last remark is shown after the proof of (2).

Suppose $\left|W: C_{W}(S)\right| \leqq\left|V: C_{V}(S)\right| \leqq 2^{2}$. Then $\left|\left[W^{*}, S, S\right]\right| \leqq 2$ for the dual module $W^{*}$ of $W$, so $G$ is a dihedral group by (1). Since $C_{W}(G) \subseteq C_{W}(P)=0$ by (2.4)-(3) and $G=\left\langle S, S^{x}\right\rangle$ for some element $x \in G$ by $(2.1)-(10)$, we have $|W| \leqq 2^{4}$. Thus, inspecting dihedral subgroups of $G L_{4}(2), G \cong \mathbf{D}_{6}$ or $\mathbf{D}_{10}$ and $|W|=2^{2}$ or $2^{4}$. This proves $(2)$.

Finally, suppose $0<|[V, S, S]| \leqq 2^{2}$ and $[V, S, S, S]=0$. Then one of the three cases (ii), (iii), (iv) of (1) occurs. Suppose $|W|=2^{4}$. Then $\left|W: C_{W}(S)\right| \leqq$ 
$|W:[W, S, S]|=2^{2}$, so $G$ is dihedral and $[V, S, S]=0$ by (2), a contradiction. Therefore $|W| \neq 2^{4}$, and hence $P \cong E s p_{27}$.

Corollary 2.13 Let $G$ be a 2-irreducible solvable group with $O_{2}(G)=1$, $S$ a Sylow 2-subgroup of $G, V$ a faithful $G F(2) G$-module, and $1 \neq t \in E \subseteq S$ and $E^{2}=1$. Suppose that $n_{*}(G) \leqq 2$, that $\left|V: C_{V}(t)\right| \leqq 2^{2}$ and, moreover, that $[V, t, E]=0$ if $t \in Z(S)$. Then one of the following holds.

(1) $G$ has a nonidentity elementary abelian 2-subgroup inducing an offending action on $\left[V, O^{2}(G)\right]$.

(2) $[V, E]=[V, t]+\left[C_{V}(t), E\right]$, and, moreover, $E=\langle t\rangle$ if $t \in Z(S)$.

Proof. Let $P=O^{2}(G)$ and $W=[V, P]$. Assume that (1) does not hold. Then we have

$$
\left|W: C_{W}(t)\right|=2^{2} \quad \text { and } \quad\left[C_{V}(P), t\right]=0 .
$$

We will prove that (2) holds, and we remark that (2) trivially holds if $E=\langle t\rangle$.

First, suppose $n(G, V) \geqq 2$. Then (2.11)-(2) shows that $t$ induces a factorwise sesquitransvection on $V$. Therefore we have

$$
O^{2}(G) \cong \mathbf{Z}_{3} \quad \text { if } \quad t \in Z(S), \quad \text { and } \quad O^{2}(G) \cong \mathbf{E}_{3^{2}} \quad \text { if } \quad t \notin Z(S) .
$$

Now, let $t \in Z(S)$ and $[V, t, E]=0$. It suffices to show that $E=\langle t\rangle$, so we may assume that $P$ is not cyclic. Thus we have $n(G, V)=1$ by the above, and then $G$ is irreducible on $W$, and $t$ induces a quasitransvection but no transvection on $W$. Therefore either $P \cong \mathbf{E}_{3^{2}}$ and $|W|=2^{4}$, or $P \cong E s p_{27}$ and $|W|=2^{6}$ by $(2.9)$ and our hypothesis. If $P \cong \mathbf{E}_{3^{2}}$, then $S \cong \mathbf{Z}_{4}$ by (2.5)-(4) because (1) does not holds, so $E=\langle t\rangle$. If $P \cong E s p_{27}$, then $E=\langle t\rangle$ by $(2.7)-(6)$.

Next, let $t \notin Z(S)$. We may assume $E \neq\langle t\rangle$, and then $\Omega_{1}(Z(S)) \subseteq\langle t, u\rangle=E$ for some $u \in t^{S}$ by (1.1). Therefore we also have

$$
\left|W: C_{W}(u)\right|=2^{2} \quad \text { and } \quad\left[C_{V}(P), u\right]=0,
$$

and hence $[V, E]=[W, E],[V, t]=[W, t]$, and $\left[C_{V}(t), E\right]=\left[C_{W}(t), E\right]$. Thus we may assume $V=W$, and then $C_{V}(P)=0$. As $t$ induces a quasitransvection on $V$, (2.9) shows that $P \cong \mathbf{E}_{3^{2}}$ or $\mathbf{E}_{5^{2}}$ if $n(G, V)=1$, and the above remark shows that $P \cong \mathbf{E}_{3^{2}}$ if $n(G, V) \geqq 2$.

Define $P_{t}=[P, t]$ and $P_{u}=[P, u]$, and regard $V$ as a $G F(2) P$-module. Let $W$ be an arbitrary homogenous component of $V$. Suppose $W \neq W^{t}$, and put $U=$ $W \oplus W^{t}$. Then we have $2^{2} \leqq|W| \leqq\left|U: C_{U}(t)\right| \leqq\left|V: C_{V}(t)\right| \leqq 2^{2}$, and so $|W|=2^{2}$ and $U \subseteq\left[V, P_{t}\right]$. Hence $V=\left[V, P_{t}\right]+\left[V, P_{u}\right] \subseteq U$ as $W^{t}=W^{u}$. Thus we have $P \cong \mathbf{E}_{3^{2}}$ and $|V|=2^{4}$, and then (1) holds by (2.5)-(5), a contradiction. Therefore $W=W^{t}$, and hence $C_{P}(W)=P_{t}$ or $P_{u}$. This shows that $V=C_{V}\left(P_{t}\right) \oplus C_{V}\left(P_{u}\right)$.

If $\left|C_{V}\left(P_{u}\right): C_{C_{V}\left(P_{u}\right)}(t)\right|=2$, then $\left|C_{V}\left(P_{t}\right)\right|=\left|C_{V}\left(P_{u}\right)\right|=2^{2}$, and so $P \cong \mathbf{E}_{3^{2}}$ and $|V|=2^{4}$, also a contradiction. Thus we have $\left|C_{V}\left(P_{u}\right): C_{C_{V}\left(P_{u}\right)}(t)\right|=2^{2}$, 
and then $\left[C_{V}\left(P_{t}\right), t\right]=0$ and, by symmetry, $\left[C_{V}\left(P_{u}\right), u\right]=0$. Hence $C_{V}(t)=$ $C_{V}\left(P_{t}\right)+C_{C_{V}\left(P_{u}\right)}(t)$, and then

$$
\left[C_{V}(t), E\right]=\left[C_{V}(t), u\right]=\left[C_{V}\left(P_{t}\right), u\right]
$$

Therefore it follows that

$$
[V, E]=[V, t]+[V, u]=[V, t]+\left[C_{V}\left(P_{t}\right), u\right]=[V, t]+\left[C_{V}(t), E\right]
$$

The next two lemmas give information on the number of noncentral chief 2 factors.

Lemma 2.14 Let $G$ be a group, $S$ a Sylow 2 -subgroup of $G, Q=O_{2}(G) \supseteq U \triangleleft$ $S, T=\left\langle U^{G}\right\rangle$, and $W=\cap U^{G}$. Then the following holds.

(1) $[T, Q]=\left\langle(U \cap[T, Q])^{G}\right\rangle=\left\langle[U, Q]^{G}\right\rangle$, and if $T \neq U$ then $\left[T, O^{2}(G)\right] \nsubseteq[T, Q]$.

(2) If $n(G, T / W) \leqq k$, then $[T, Q ; k] \leqq U$.

(3) There exists a normal subgroup $N$ of $G$ such that $W \subseteq N \subseteq T$ and $T / N$ is a noncentral chief factor of $G$.

Proof. Let $L=[T, Q]$ and $M=\left\langle[U, Q]^{G}\right\rangle$. Then $M \subseteq\left\langle(U \cap L)^{G}\right\rangle \leqq L$ because $[U, Q] \subseteq L \cap M$. Since $[U M, Q] \subseteq M$ and $\left\langle(U M)^{G}\right\rangle=T$, we have $L=$ $[T, Q] \subseteq M$, so $L=\left\langle(U \cap L)^{G}\right\rangle=M$. Suppose $\left[T, O^{2}(G)\right] \subseteq L$. Then, since $U L \triangleleft S, T=\left\langle U^{G}\right\rangle \subseteq U L$, so we have $T=U L$. This shows that $T=U$, so (1) holds.

Part (2) holds for $k=0$ by (1). Suppose $k \geqq 1$, and define $T_{0}=L W$ and $U_{0}=$ $U \cap T_{0}$. Then $T_{0}=\left\langle U_{0}{ }^{G}\right\rangle$ and $n\left(G, T / T_{0}\right) \geqq 1$ by $(1)$, and so $n\left(G, T_{0} / W\right) \leqq k-1$. Thus $\left[T_{0}, Q ; k-1\right] \subseteq U$ by the inductive hypothesis, and hence $[T, Q ; k] \subseteq U$.

To prove (3), let $\widetilde{T}=T / T_{0}$ and regard $\widetilde{T}$ as a $G F(2) G$-module with $Q \subseteq$ $C_{G}(\widetilde{T}) \nsupseteq O^{2}(G)$. Then we have $\widetilde{T}=\left[\widetilde{T}, O^{2}(G)\right] \times C_{\widetilde{T}}\left(O^{2}(G)\right) \neq C_{\widetilde{T}}\left(O^{2}(G)\right)$ by (2.4)-(3). Choose a subgroup $N$ so that $T_{0} \subseteq N \subseteq T$ and $\tilde{N}$ is a maximal submodule of $\widetilde{T}$ containing $C_{\widetilde{T}}\left(O^{2}(G)\right)$. Then $N$ has the desired property.

Lemma 2.15 Let $G$ be a 2-irreducible solvable group, $S$ a Sylow 2-subgroup, $Q=O_{2}(G) \supseteqq U \triangleleft S, T=\left\langle U^{G}\right\rangle$, and $W=\cap U^{G}$. Suppose $n(G, T / W)=r$ and that $\left|C_{V}(S)\right|=2$ for all noncentral chief factors $V$ of $G$ within $T / W$. Then $\left|C_{U / W}(R)\right| \leqq 2^{r}$ for all normal subgroups $R$ of $S$ with $S=Q R$.

Proof. We may assume that $[U, R] \subseteq W$. Define the subgroups $K_{m}$ and $L_{m}(m \geqq 0)$ as follows:

$$
K_{0}=L_{0}=T \text {; }
$$


if $L_{m-1} \neq W$, then choose $K_{m}$ so that $W \subseteq K_{m} \subseteq L_{m-1}$ and that $L_{m-1} / K_{m}$ is a noncentral chief factor of $G$ (this is possible by (2.14)-(3)), and put $L_{m}=\left\langle\left(U \cap K_{m}\right)^{G}\right\rangle$;

if $L_{m-1}=W$, then $K_{m}=L_{m}=K_{m+1}=L_{m+1}=\cdots=W$.

Let $n$ be a positive integer so that $L_{n-1} \neq W=L_{n}$. Then we have $n \leqq r$. Therefore, since $\left|U \cap L_{m-1}: U \cap L_{m}\right|=2(1 \leqq m \leqq n)$ by our hypothesis, we have $|U: W|=2^{n} \leqq 2^{r}$.

\section{$\S 3$. Amalgams of solvable groups} ditions:

In this section, we study the amalgams $(X, S, Y)$ satisfying the following con-

(a) $S$ is a common Sylow 2-subgroup of $X$ and $Y$;

(b) both $X$ and $Y$ are 2-irreducible;

(c) no nonidentity subgroup of $S$ is normal both in $X$ and in $Y$;

(d) $\quad C_{X}\left(O_{2}(X)\right) \subseteq O_{2}(X)$ and $C_{Y}\left(O_{2}(Y)\right) \subseteq O_{2}(Y)$;

(f) both $X$ and $Y$ are solvable;

(g) $S=\left[O_{2}(X), O^{2}(X)\right]\left(O_{2}(X) \cap O_{2}(Y)\right)\left[O_{2}(Y), O^{2}(Y)\right]$.

We will use the following notation throughout this section:

$$
\begin{aligned}
& Q=O_{2}(X) ; \quad R=O_{2}(Y) ; \\
& Z=\Omega_{1}(Z(S)) ; \quad V=\left\langle Z^{X}\right\rangle ; \quad W=\left\langle Z^{Y}\right\rangle ; \\
& \pi(X)=\{2, q\} ; \quad \pi(Y)=\{2, r\} .
\end{aligned}
$$

(3.1) If a subgroup $U$ of $S$ is normalized both by a Sylow q-subgroup $K$ of $X$ and by a Sylow $r$-subgroup $L$ of $Y$, then $U=1$.

Proof. Since $X=S K=K S$ and $Y=S L=L S,\left\langle U^{S}\right\rangle$ is normal both in $X$ and in $Y$. Thus $U \subseteq\left\langle U^{S}\right\rangle=1$ by (c).

(3.2) The following holds.

(1) $S=Q R \neq Q=\left[Q, O^{2}(X)\right](Q \cap R)$.

(2) $\quad\left[Q, O^{2}(X)\right] \nsubseteq Q \cap R \nrightarrow X$.

(3) $\quad Q=\left(R^{x} \cap Q\right)(Q \cap R)$ and $X=\left\langle R^{x}, R\right\rangle=\langle R, x\rangle$ if $X=\langle S, x\rangle$.

(4) The statements in (1)-(3) remain true if we replace $X, Q, R$ by $Y, R$, $Q$, respectively.

Proof. First, (4) holds by symmetry of $X$ and $Y$. We have $S=Q R$ and $Q=\left[Q, O^{2}(X)\right](Q \cap R)$ by $(\mathrm{g})$, and $Q \neq S \neq R$ by (2.3). Therefore $Q \neq Q \cap R$, and hence $\left[Q, O^{2}(X)\right] \nsubseteq Q \cap R$.

Choose $x \in X$ so that $X=\langle S, x\rangle$. Then $X=\left\langle S^{x}, S\right\rangle=\left\langle(Q R)^{x}, Q R\right\rangle=$ $\left\langle R^{x}, Q, R\right\rangle$ by $(2.1)-(10)$. Let $T=\left(R^{x} \cap Q\right)(R \cap Q)$. Since $\left[Q, R^{x}\right] \subseteq Q \cap R^{x} \subseteq T \supseteqq$ 
$Q \cap R \supseteqq[Q, R],[Q, X] \subseteq[Q, Q] T$, and hence $Q=\left[Q, O^{2}(X)\right](Q \cap R) \subseteq[Q, Q] T$. This yields that $Q=T$, and so $Q \cap R \Varangle X$ because $Q \neq Q \cap R$. Therefore $X=$ $\left\langle R^{x}, Q, R\right\rangle=\left\langle R^{x},\left(R^{x} \cap Q\right)(Q \cap R), R\right\rangle=\left\langle R^{x}, R\right\rangle=\langle R, x\rangle$.

(3.3) Let $Y \triangleright U \subseteq Q$. Suppose that there exists a normal elementary abelian subgroup $T / U$ of $S / U$ not contained in $R / U$. Then $n_{*}(X)=1$ and $|S: Q|=2$.

Proof. Take $y \in Y$ so that $Y=\langle S, y\rangle$. Since $R \supseteqq T^{y} \triangleleft S^{y}, \Omega_{1}\left(Z\left(S^{y} / R\right)\right) \subseteq$ $T^{y} R / R$, so $Y=\left\langle S, T^{y}\right\rangle$ by (2.1)-(10). Thus $Y=\left\langle Q, T^{y}\right\rangle$ by (3.2)-(3), and so, $\left[R, O^{2}(Y)\right] \subseteq[R, Y] \subseteq[R, Q]\left[R, T^{y}\right] \subseteq(Q \cap R)\left(R \cap T^{y}\right)$. Therefore $S=$ $Q\left[R, O^{2}(Y)\right]=Q\left(R \cap T^{y}\right)$, and hence $S / Q$ is elementary abelian. Thus (2.1)-(7) shows that $n_{*}(X)=1$ and $|S: Q|=2$.

(3.4) Suppose that either $V \nsubseteq R$ or $W \varsubsetneqq Q$. Then $n_{*}(X)=1=n_{*}(Y)$.

Proof. We may assume, by symmetry, that $V \nsubseteq R$. Then $n_{*}(X)=1$ and $|R: Q \cap R|=|S: Q|=2$ by (3.3). Thus $V$ induces a factorwise transvection on $R$ because $[Q \cap R, V]=1$. Since $S=Q R \subseteq C_{Y}(V) R, V R / R \subseteq \Omega_{1}(Z(S / R))$, and hence $(2.11)-(1)$ shows that $O^{2}(Y / R)=O^{2}\left(Y / R C_{Y}(R)\right) \cong \mathbf{Z}_{3}$. Therefore $n_{*}(Y)=1$ by $(2.1)-(9)$.

(3.5) If $\langle V, W\rangle \subseteq Q \cap R$, then either $Z \subseteq Z(X)$ or $Z \subseteq Z(Y)$.

Proof. Suppose $\langle V, W\rangle \subseteq Q \cap R$, and that $Z(X) \nsupseteq Z \varsubsetneqq Z(Y)$. By symmetry, we may assume $Q(K(S)) \Varangle X$. Then $(2.2)$ shows that $\left[Q, O^{2}(X)\right] \subseteq V \subseteq Q \cap R$, contrary to $(3.2)-(2)$.

(3.6) If $Z \subseteq Z(Y)$, then $V=\left[V, O^{2}(X)\right]$ and $C_{V}(X)=1$.

Proof. Since $C_{V}(S) \cap C_{V}\left(O^{2}(X)\right)=C_{Z}(X)=1$ by (c), we have $C_{V}(X)$ $\subseteq C_{V}\left(O^{2}(X)\right)=1$. Thus we have $V=\left[V, O^{2}(X)\right]$ by (2.4)-(3), regarding $V$ as a $G F(2) X$-module.

(3.7) Suppose $Z \subseteq Z(Y)$. If $\left\langle V^{Y}\right\rangle$ is not abelian, then $n_{*}(X)=1=n_{*}(Y)$.

Proof. We may assume $V \subseteq R$ by (3.4), and so $\left[Q / V, O^{2}(X)\right] \neq 1$ by (3.2)-(2). Take $y \in Y$ so that $\left[V, V^{y}\right] \neq 1$. We may assume $\left|V: V \cap Q^{y}\right| \leqq \mid V$ : $V \cap Q^{y^{-1}}|=| V^{y}: V^{y} \cap Q \mid$, replacing $y$ by $y^{-1}$ if necessary. Let $\bar{X}=X / C_{X}(V)$, and regard $V$ as a faithful $G F(2) \bar{X}$-module. Then we have

$$
C_{V}(\bar{S}) \varsubsetneqq C_{V}(\bar{X})
$$

by (3.6). Moreover, since $Q \subseteq C_{X}(V) \nsupseteq O^{2}(X), \bar{X}$ is a 2-irreducible solvable group with $O_{2}(\bar{X})=1$ and $V^{y} \cap Q=C_{V^{y}}(V)$ by (2.4)-(1). Similarly, regarding $V^{y}$ as a $G F(2) X^{y}$-module, we have $V \cap Q^{y}=C_{V}\left(V^{y}\right)=C_{V}\left(\overline{V^{y}}\right)$, and hence $\left|V: C_{V}\left(\overline{V^{y}}\right)\right| \leqq\left|\overline{V^{y}}\right| \neq 1$. 
Let $\bar{A}$ be a nonidentity elementary abelian 2-subgroup of $\bar{X}$ that is minimal under the condition $\left|V: C_{V}(\bar{A})\right| \leqq|\bar{A}| \neq 1$. We will prove that

$$
|\bar{A}| \cdot\left|C_{V}(\bar{A})\right| \geqq|\bar{B}| \cdot\left|C_{V}(\bar{B})\right| \text { for all subgroups } \bar{B} \text { of } \bar{A} \text {. }
$$

This is true if $\bar{B}=1$ or $\bar{A}$. Suppose $1 \neq \bar{B} \neq \bar{A}$. Then the minimality of $\bar{A}$ forces that $\left|V: C_{V}(\bar{B})\right|>|\bar{B}|$, and hence $|\bar{A}| \cdot\left|C_{V}(\bar{A})\right| \geqq|V|>|\bar{B}| \cdot\left|C_{V}(\bar{B})\right|$.

We have shown that the pair $(\bar{X}, V)$ satisfies Hypothesis II of [5]. Therefore there exists, by 5.1 of [5], a normal subgroup $\bar{N}$ of $\bar{X}$ such that $\bar{X}=\overline{S N}$ and $\bar{N} \cong \Sigma_{3} \times \cdots \times \Sigma_{3}$ (k copies), and, moreover, $V=[V, \bar{N}]$ by (3.6). Since $V^{y} \triangleleft R$ and $S=Q R$, we can choose $t \in V^{y}-Q$ so that $t Q \in Z(S / Q)$. Thus we have

$$
2^{k}=\left|V: C_{V}(\bar{t})\right| \leqq\left|V: C_{V}\left(\overline{V^{y}}\right)\right| \leqq\left|\overline{V^{y}}\right|
$$

by 5.6 of $[\mathbf{5}]$. Since $\left[V, V^{y}, V^{y}\right]=1,5.7$ of $[\mathbf{5}]$ shows that $\left|\overline{V^{y}}\right|=2^{k}$ and that $C_{\bar{X}}\left(\overline{V^{y}}\right)=\overline{V^{y}}$, and then 5.8 of $[\mathbf{5}]$ shows that $\overline{V^{y}}=\overline{S \cap N}$. Therefore $\left|V: V \cap Q^{y}\right|=\left|V^{y}: V^{y} \cap Q\right|=\left|V: V \cap Q^{y^{-1}}\right|$. This shows that $\overline{V^{z}}=\overline{S \cap N}$ for all $z \in Y$ with $\left[V, V^{z}\right] \neq 1$, and hence $\bar{U}=\overline{S \cap N}$ and $U=(U \cap Q) V^{y}=$ $(U \cap Q) V^{y^{-1}}=\left(U \cap Q^{y}\right) V$, where $U=\left\langle V^{Y}\right\rangle$. Therefore $\left[U, V^{y}\right]=\left[V, V^{y}\right] \subseteq V$, and so $Q / V \nsupseteq V V^{y} / V \subseteq \Omega_{1}(Z(U / V)) \triangleleft S / V$. Thus $n_{*}(Y)=1$ and $\left|Q^{y}: R \cap Q^{y}\right|=$ $\left|S^{y}: R\right|=2$ by $(3.3)$.

Note that $V \cong Q \cap R \cong C_{S^{y} \bmod Q^{y}}(V)=V Q^{y}$ as above. Thus $Q \cap R=$ $V\left(Q \cap R \cap Q^{y}\right)$, and hence $t$ induces a factorwise transvection on $Q / V$. Therefore $O^{2}\left(X / Q C_{X}(Q / V)\right) \cong \mathbf{Z}_{3}$ by $(2.11)-(1)$, and so $n_{*}(X)=1$ by $(2.1)-(9)$.

(3.8) Let $X \triangleright A \subseteq R$, and suppose $n(Y, B / C)=1$ where $B=\left\langle A^{Y}\right\rangle$ and $C=\cap A^{Y}$. Let $D \triangleleft Y$ and $E \triangleleft X$ so that

$$
\left[D, O^{2}(Y)\right] \subseteq B \subseteq D \subseteq R, \quad A \subseteq E \subseteq Q, \text { and } B \varsubsetneqq E .
$$

Then $\left[E \cap D,\left[R, O^{2}(Y)\right]\right] \subseteq A$. In particular, $\left[E, O^{2}(X)\right] \subseteq A$ if $E \subseteq D$.

Proof. We may assume $D=(E \cap D) B=\left\langle(E \cap D)^{Y}\right\rangle$. Let $P=\left[R, O^{2}(Y)\right]$, $F=\left[D, O^{2}(Y)\right] C$, and $J=[D, P] C$. Since $J \subseteq[D, R] C$, we have $\left[D, O^{2}(Y)\right] \nsubseteq J$ by (2.14)-(1), and so $J \subset F \subseteq B$ and $\left[J, O^{2}(Y)\right] \subseteq C$ because $n(Y, D / C)=1$. Since $[B, R] \subseteq C$ by $(2.14)-(2)$, we have $B / C=F / C \times C_{B / C}\left(O^{2}(Y)\right)$ by $(2.4)-(3)$, regarding $B / C$ as a $G F(2) Y$-module. Thus $C_{F / C}\left(O^{2}(Y)\right)=1$ and so $J \subseteq C$. This shows that $[E \cap D, P] \subseteq J \subseteq C \cong A$. Suppose $E \subseteq D$. Since $P \varsubsetneqq Q$ by (3.2)-(3), $X$ has no noncentral chief factor within $E / A$, and hence $\left[E, O^{2}(X)\right] \subseteq A$.

(3.9) Let $X \triangleright A \subseteq R$, and suppose $n(Y, B / C)=1$ and $(Y, B / C) \ll$ $\left(O_{4}^{+}(2), G F(2)^{4}\right)$ where $B=\left\langle A^{Y}\right\rangle$ and $C=\cap A^{Y}$. Assume $B \subseteq Q$, and define $E=\cap B^{X}, D=\left\langle B^{X}\right\rangle$, and $D^{(n)}=[D, Q ; n] A$ for $n \geqq 0$. Then the following holds. 
(1) $\quad 2^{4} \leqq|B: C| \leqq 2^{4} \cdot|A: C|$.

(2) If $|B: A|=2^{n}$, then $\left|B \cap D^{(k-1)}: B \cap D^{(k)}\right|=2$ for $1 \leqq k \leqq n$.

(3) $|E: A| \leqq 2$, and $D^{(m-1)} \neq E=D^{(m)}$ if $|B: E|=2^{m}$.

Proof. Let $M=\left[B, O^{2}(Y)\right] C$. Then $B=A M$ and, moreover, $[B, R] \subseteq C$ by $(2.14)-(2)$ as $n(Y, B / C)=1$. Thus we have $B / C=M / C \times C_{B / C}\left(O^{2}(Y)\right)$ by (2.4)-(3), and hence $(Y, M / C) \ll\left(O_{4}^{+}(2), G F(2)^{4}\right)$, and $|B: C|=|B: M| \cdot \mid M:$ $C|=| A: A \cap M|\cdot| M: C|\leqq| A: A \cap M \mid \cdot 2^{4}$.

Let $|B: A|=2^{n}$ and $|B: E|=2^{m}$. Since $B / A$ is isomorphic, as $G F(2) S$ modules, to a factor module of $M / C$, we have

$$
|[B, Q ; k-1] A:[B, Q ; k] A|=2 \quad(1 \leqq k \leqq n),
$$

by (2.5)-(3). Thus $\left|B \cap D^{(k-1)}: B \cap D^{(k)}\right|=2(1 \leqq k \leqq n)$, and, in particular, $D^{(m-1)} \neq D^{(m)}$. Since $[E, R] \subseteq A$ and $X=\left\langle R^{X}\right\rangle,[E, S] \subseteq[E, X] \subseteq A$, and so $|E: A| \leqq 2$.

(3.10) Let $X \triangleright A \neq 1$ and $Y \triangleright B, C$ with $C \subseteq A \subseteq B \subseteq R$. Suppose $O^{2}\left(Y / R C_{Y}(B / C)\right)$ is elementary abelian and nonidentity. Then the following holds.

(1) Let $T$ be a 2-subgroup of $Y$. If $Y \neq\langle S, T\rangle$, then $T \subseteq S^{y}$ and $A=A^{y}$ for some $y \in Y$.

(2) Let $z \in Y$. Then $A \neq A^{z}$ if and only if $Y=\left\langle S, S^{z}\right\rangle$.

Proof. Let $M=S C_{Y}(B / C)$. Then $M$ is the unique maximal subgroup of $Y$ containing $S$ by $(2.1)-(8)$.

Suppose $Y \neq\langle S, T\rangle$ for a 2-subgroup $T$ of $Y$. Then $T \subseteq M$, so there exists an element $y \in M$ such that $T \subseteq S^{y}$. We have $A=A^{y}$ by the definition of $M$. Hence (1) holds.

Let $z \in Y$. If $A=A^{z}$, then $A \triangleleft\left\langle X, S^{z}\right\rangle$, so $Y \neq\left\langle S, S^{z}\right\rangle$ by (c). Suppose $Y \neq\left\langle S, S^{z}\right\rangle$. Then $S^{z}=S^{y}$ and $A=A^{y}$ for some $y \in Y$ by (1). Since $S \nless Y$ by (3.2)-(1), we have $z y^{-1} \in N_{Y}(S) \subseteq M$, and so $A^{z y^{-1}}=A$. Thus $A^{z}=A^{y}=A$, proving (2).

\section{$\S 4$. The coset graph}

Throughout the remainder of this paper, suppose the amalgam $(X, S, Y)$ satisfies the hypothesis of Theorem C.

First, we will define the coset graph $\Gamma=\Gamma(X, S, Y)$ associated with the amalgam $(X, S, Y)$. Let $G$ be the amalgamated product of $X$ and $Y$ over $S$, and embed $S, X, Y$ into $G$. Note that $S=X \cap Y$ in $G$ by the uniqueness of the standard forms of elements in an amalgamated product. The set $V(\Gamma)$ of vertices of $\Gamma$ consists of the right cosets of $X$ and $Y$ in $G$, and two vertices are adjacent if and only if they are distinct and have a nonempty intersection. Then $G$ acts on $\Gamma$ by right multipli- 
cation. We call a set of two adjacent vertices an edge, and denote by $E(\Gamma)$ the set of edges of $\Gamma$.

(4.1) The group $G$ acts transitively on $E(\Gamma)$.

Proof. Let $\{\xi, \eta\} \in E(\Gamma)$. We may assume $\xi=X g$ and $\eta=Y g^{\prime}$ for some elements $g, g^{\prime} \in G$. Since $X g \cap Y g^{\prime} \neq \phi$, there exists an element $h \in G$ such that $\left(X g \cap Y g^{\prime}\right) h \cap S \neq \phi$, and hence $X g h \cap S \neq \phi \neq Y g^{\prime} h \cap S$. Therefore we have $X g h=X$ and $Y g^{\prime} h=Y$, and so $\{\xi, \eta\}^{h}=\{X, Y\}$.

We denote by $G_{\xi}$ the stabilizer of $\xi$ in $G$ for all $\xi \in V(\Gamma)$. Then (4.1) shows that the amalgam $(X, S, Y)$ is isomorphic to an amalgam $\left(G_{\xi}, G_{\xi \eta}, G_{\eta}\right)$ for all edges $\{\xi, \eta\} \in E(\Gamma)$ where $G_{\xi \eta}=G_{\xi} \cap G_{\eta}$, and hence $\left(\mathrm{f}_{2}\right)$ forces that $n_{*}\left(G_{\xi}\right) \leqq f\left(G_{\xi}\right) \leqq 2 \geqq f\left(G_{\eta}\right) \geqq n_{*}\left(G_{\eta}\right)$.

For $\xi \in V(\Gamma)$, let $\Delta(\xi)=\Delta_{\Gamma}(\xi)$ be the set of vertices of $\Gamma$ adjacent to $\xi$, and put $Q_{\xi}=O_{2}\left(G_{\xi}\right)$ and $V_{\xi}=\left\langle\Omega_{1}(Z(T)) \mid T \in \operatorname{Syl}_{2}\left(G_{\xi}\right)\right\rangle$. Then $G_{\xi}$ is transitive on $\Delta(\xi)$ by (4.1), and hence $\operatorname{Syl}_{2}\left(G_{\xi}\right)=\left\{G_{\xi \eta} \mid \eta \in \Delta(\xi)\right\}$. Thus we have

$$
V_{\xi}=\left\langle\Omega_{1}\left(Z\left(G_{\xi \eta}\right)\right) \mid \eta \in \Delta(\xi)\right\rangle \text {. }
$$

A path of length $n$ in $\Gamma$ is a sequence $\mathcal{S}=\left(\xi_{0}, \xi_{1}, \ldots, \xi_{n}\right)$ of $n+1$ vertices of $\Gamma$ such that $\left\{\xi_{i-1}, \xi_{i}\right\} \in E(\Gamma)$ for $1 \leqq i \leqq n$.

(4.2) The graph $\Gamma$ is connected, that is, every pair of vertices is joined by a path.

Proof. Let $\Gamma_{0}$ be a connected component of $\Gamma$ containing the edge $\{X, Y\}$. Then $G$ leaves $\Gamma_{0}$ invariant because $G=\langle X, Y\rangle$. Thus every edge of $\Gamma$ is contained in $\Gamma_{0}$ by (4.1), and hence $\Gamma=\Gamma_{0}$.

Let $\lambda, \mu \in V(\Gamma)$. The distance $d(\lambda, \mu)=d_{\Gamma}(\lambda, \mu)$ of $\lambda, \mu \in V(\Gamma)$ is the minimum length of the paths from $\lambda$ through $\mu$ in $\Gamma$. We will define $b=b(X, S, Y)$ to be the minimum distance $d(\alpha, \delta)$ of $\alpha, \delta \in V(\Gamma)$ under the condition that $V_{\alpha} \varsubsetneqq Q_{\delta}$.

(4.3) We have $1 \leqq b<\infty$.

Proof. Note that $\bigcap_{\xi \in V(\Gamma)} Q_{\xi} \sqsubseteq \bigcap_{\xi \in V(\Gamma)} G_{\xi}=1$ by (c). Thus there exists a pair $(\alpha, \delta)$ of vertices of $\Gamma$ such that $V_{\alpha} \nsubseteq Q_{\delta}$. Hence $b$ is well-defined and $b<\infty$ because $d(\alpha, \delta)<\infty$ by $(4.2)$. Also, we have $1 \leqq b$ because $V_{\xi}=\left\langle\Omega,\left(Z\left(G_{\xi \eta}\right)\right)^{G_{\xi}}\right\rangle$ by $(*)$ and $\Omega_{1}\left(Z\left(G_{\xi \eta}\right)\right) \subseteq C_{G_{\xi}}\left(Q_{\xi}\right) \subseteq Q_{\xi}$ by $(\mathrm{d})$.

Now, define

$$
\begin{aligned}
\mathcal{A} & =\left\{\alpha \in V(\Gamma) \mid V_{\alpha} \nsubseteq Z\left(G_{\alpha}\right)\right\}, \\
\mathcal{B} & =V(\Gamma)-\mathcal{A}, \quad \text { and } \\
\mathcal{C} & =\left\{(\alpha, \delta) \mid \alpha, \delta \in V(\Gamma), V_{\alpha} \nsubseteq Q_{\delta}, d(\alpha, \delta)=b\right\} .
\end{aligned}
$$


(4.4) Let $\{\xi, \eta\} \in E(\Gamma)$, and suppose $\eta \in \mathcal{B}$. Then $\xi \in \mathcal{A}, V_{\eta}=\Omega_{1}\left(Z\left(G_{\xi \eta}\right)\right)$, and $V_{\xi}=\left\langle V_{\eta} G_{\xi}\right\rangle$.

Proof. We have $V_{\eta}=\left\langle\Omega_{1}\left(Z\left(G_{\xi \eta}\right)\right)^{G_{\eta}}\right\rangle=\Omega_{1}\left(Z\left(G_{\xi \eta}\right)\right)$ by $(*)$. Hence $\xi \in \mathcal{A}$ by (c), and $V_{\xi}=\left\langle\Omega_{1}\left(Z\left(G_{\xi \eta}\right)\right)^{G_{\xi}}\right\rangle=\left\langle V_{\eta}{ }^{G}\right\rangle$ by $(*)$ and the above.

(4.5) If $(\alpha, \delta) \in \mathcal{C}$, then $\alpha \in \mathcal{A}$.

Proof. Let $(\alpha, \beta, \ldots, \delta)$ be a path of length $b$ from $\alpha$ through $\delta$. Suppose $\alpha \in \mathcal{B}$. Then $V_{\alpha} \subseteq V_{\beta}$ by $(4.4)$, so $V_{\beta} \varsubsetneqq Q_{\delta}$ and $d(\beta, \delta)<b$, contrary to the minimality of $b$.

(4.6) Suppose $b \geqq 2$. Then either

$$
\xi \in \mathcal{A} \quad \text { and } \quad \eta \in \mathcal{B}, \quad \text { or } \quad \eta \in \mathcal{A} \quad \text { and } \quad \xi \in \mathcal{B}
$$

for all $\{\xi, \eta\} \in E(\Gamma)$.

Proof. Let $\{\xi, \eta\} \in E(\Gamma)$. Since $b \geqq 2$, we have $\left\langle V_{\xi}, V_{\eta}\right\rangle \subseteq Q_{\xi} \cap Q_{\eta}$, so either $\Omega_{1}\left(Z\left(G_{\xi \eta}\right)\right) \subseteq Z\left(G_{\xi}\right)$ or $\Omega_{1}\left(Z\left(G_{\xi \eta}\right)\right) \subseteq Z\left(G_{\eta}\right)$ by (3.5). This yields that either $\xi \in \mathcal{B}$ or $\eta \in \mathcal{B}$, and so the assertion follows from (4.4).

For the proof of Theorem C, it suffices to prove that $n_{*}\left(G_{\xi}\right)=1=n_{*}\left(G_{\eta}\right)$ for some $\{\xi, \eta\} \in E(\Gamma)$ by (4.1). We now obtain the following theorem.

Theorem 4.7 Suppose $b \leqq 2$. Then $n_{*}\left(G_{\xi}\right)=1=n_{*}\left(G_{\eta}\right)$ for some $\{\xi, \eta\} \in$ $E(\Gamma)$.

Proof. Let $(\alpha, \delta) \in \mathcal{C}$. We have $V_{\alpha} \nsubseteq Q_{\delta}$ and $d(\alpha, \delta)=b$ by the definition of $\mathcal{C}$.

Suppose first that $b=1$. Then $\{\alpha, \delta\} \in E(\Gamma)$, and hence $n_{*}\left(G_{\alpha}\right)=1=n_{*}\left(G_{\delta}\right)$ by (3.4).

Suppose next that $b=2$, and take $\beta \in V(\Gamma)$ so that $\beta \in \Delta(\alpha) \cap \Delta(\delta)$. Then (4.3) and (4.4) show that $\alpha \in \mathcal{A} \ni \delta$ and $\beta \in \mathcal{B}$. Since $V_{\alpha} \subseteq Q_{\beta}$ by the minimality of $b$ and $C_{G_{\beta \delta}}\left(V_{\delta}\right)=Q_{\delta}$ by $(2.4)-(1)$, we have $\left[V_{\alpha}, V_{\delta}\right] \neq 1$, and so $\left\langle V_{\alpha} G_{\beta}\right\rangle$ is nonabelian. Thus $n_{*}\left(G_{\alpha}\right)=1=n_{*}\left(G_{\beta}\right)$ by $(3.7)$.

In the remaining four sections, we treat the case where $b \geqq 3$. By (4.6), we can identify an edge with an ordered pair $\langle\xi, \eta\rangle$ of adjacent vertices with $\xi \in \mathcal{A}$ and $\eta \in \mathcal{B}$. Since $G_{\xi} \not G_{\eta}$ for all $\xi \in \mathcal{A}$ and $\eta \in \mathcal{B},(4.1)$ shows that $G$ acts transitively on $\mathcal{A}$ and $\mathcal{B}$, respectively. Therefore, for the proof of Theorem $\mathrm{C}$, it suffices to prove that $n_{*}\left(G_{\xi}\right)=1=n_{*}\left(G_{\eta}\right)$ for some $\xi \in \mathcal{A}$ and some $\eta \in \mathcal{B}$.

\section{$\S 5 . \quad$ Even case}

In this section, we consider the case where $b$ is even and $b \geqq 4$. 
Theorem 5.1 Let $\xi \in \mathcal{A}$ and $\eta \in \mathcal{B}$. If $b$ is even and $b \geqq 4$, then $n_{*}\left(G_{\xi}\right)=$ $1=n_{*}\left(G_{\eta}\right)$.

Proof. Pick $(\alpha, \delta) \in \mathcal{C}$, and denote by $(\alpha, \alpha+1, \alpha+2, \ldots, \delta-1, \delta)$ a path of length $b$ from $\alpha$ through $\delta$. Then $\alpha \in \mathcal{A}, \alpha+1 \in \mathcal{B}, \alpha+2 \in \mathcal{A}, \ldots, \delta-1 \in \mathcal{B}, \delta \in \mathcal{A}$ by (4.5) and (4.6). Also, we have $V_{\delta} \subseteq Q_{\alpha+1} \subseteq G_{\alpha, \alpha+1}$ (resp. $V_{\alpha} \subseteq Q_{\delta-1} \subseteq G_{\delta-1, \delta}$ ) by the minimality of $b$, and so $V_{\delta} \cap Q_{\alpha}=C_{V_{\delta}}\left(V_{\alpha}\right)$ (resp. $V_{\alpha} \cap Q_{\delta}=C_{V_{\alpha}}\left(V_{\delta}\right)$ ) by (2.4)-(1), regarding $V_{\alpha}$ (resp. $V_{\delta}$ ) as a $G F(2) G_{\alpha^{-}}$(resp. $G F(2) G_{\delta^{-}}$) module. Since $V_{\alpha} \nsubseteq Q_{\delta}$ by the definition of $\mathcal{C}$, we have $\left[V_{\alpha}, V_{\delta}\right] \neq 1$, so $V_{\delta} \nsubseteq Q_{\alpha}$. Now, by symmetry of $\alpha$ and $\delta$, we may assume $\left|V_{\alpha}: V_{\alpha} \cap Q_{\delta}\right| \leqq\left|V_{\delta}: V_{\delta} \cap Q_{\alpha}\right| \neq 1$, and hence

$$
\left|V_{\alpha}: C_{V_{\alpha}}\left(\bar{V}_{\delta}\right)\right| \leqq\left|\bar{V}_{\delta}\right| \neq 1
$$

where $\bar{G}_{\alpha}=G_{\alpha} / C_{G_{\alpha}}\left(V_{\alpha}\right)$. We will prove that $n_{*}\left(G_{\alpha}\right)=1=n_{*}\left(G_{\alpha+1}\right)$ in a series of lemmas.

For a while, regard $V_{\alpha}$ as a faithful $G F(2) \bar{G}_{\alpha}$-module.

(1) $O^{2}\left(\bar{G}_{\alpha}\right) \cong \mathbf{E}_{3^{k}}$ and $\left|V_{\alpha}\right|=2^{2 k}$, where $k=1$ or 2 .

Proof. By (2.4)-(1) and (3.6), $\bar{G}_{\alpha}$ is a 2-irreducible solvable group with $O_{2}\left(\bar{G}_{\alpha}\right)=1$ and $V_{\alpha}=\left[V_{\alpha}, O^{2}\left(\bar{G}_{\alpha}\right)\right]$. Since $n_{*}\left(\bar{G}_{\alpha}\right) \leqq n_{*}\left(G_{\alpha}\right) \leqq f\left(G_{\alpha}\right) \leqq 2$, the assertion follows from $(*)$ together with $(2.10)-(1)$.

Throughout the remainder of this proof of (5.1), let the letter $k$ have the same meaning as in (1).

Now, we will introduce the following notation.

Notation. Let $\langle\xi, \eta\rangle \in E(\Gamma)$.

$$
\begin{aligned}
& Z_{\xi \eta}=\Omega_{1}\left(Z\left(G_{\xi \eta} \bmod Q_{\xi}\right)\right), \\
& E_{\xi \eta}=C_{G_{\xi \eta} \bmod Q_{\xi}}\left(C_{V_{\xi}}\left(Z_{\xi \eta}\right)\right), \\
& U_{\eta}=\left\langle V_{\xi}{ }^{G_{\eta}}\right\rangle, \\
& U_{\eta}{ }^{(n)}=\left[U_{\eta}, Q_{\eta} ; n\right] \text { for } n \geqq 0, \\
& W_{\xi}=\cap U_{\eta}{ }^{G_{\xi}}, \text { and } \\
& T_{\xi}=\left\langle U_{\eta}{ }^{G_{\xi}}\right\rangle .
\end{aligned}
$$

(2) If $k=2$, then $Z_{\alpha, \alpha+1} \nsupseteq V_{\delta} \subseteq E_{\alpha, \alpha+1}$, and $\bar{E}_{\alpha, \alpha+1}$ is the unique fours subgroup of $\bar{G}_{\alpha, \alpha+1}$ acting quadratically on $V_{\alpha}$.

Proof. This follows from (2.5)-(4)(5).

(3) Let $\langle\xi, \eta\rangle \in E(\Gamma)$. Then

$$
U_{\eta}^{(2 k-1)}=V_{\eta}=\cap V_{\xi}^{G_{\eta}} .
$$


Proof. Let $Z=\cap V_{\xi}^{G_{\eta}}$. Since $G_{\eta}=\left\langle Q_{\xi}{ }^{G_{\eta}}\right\rangle$ by $(3.2)-(3)$, we have $V_{\eta} \subseteq Z \subseteq C_{V_{\varepsilon}}\left(G_{\eta}\right) \subseteq V_{\eta}$.

Suppose first that $k=1$. Then we have $\left|V_{\xi}: V_{\eta}\right|=2$ by (1), and hence $\left[V_{\xi}, Q_{\eta}\right]=V_{\eta}$ because $Q_{\eta} \nsubseteq Q_{\xi}=C_{G_{\xi \eta}}\left(V_{\xi}\right)$ by $(3.2)-(1)$ and (2.4)-(1). Suppose next that $k=2$. Then (2.5)-(4) shows that $\left[V_{\xi}, Q_{\eta} ; 3\right]=\left[V_{\xi}, G_{\xi \eta} ; 3\right]=V_{\eta}$. Therefore we have $\left[V_{\xi}, Q_{\eta} ; 2 k-1\right]=V_{\eta}$, and so $\left[U_{\eta}, Q_{\eta} ; 2 k-1\right]=V_{\eta}$ by $(2.14)-(1)$.

Let $\lambda \in \Delta(\delta)$ so that $G_{\delta}=\left\langle V_{\alpha}, G_{\delta \lambda}\right\rangle$. We have $G_{\delta}=\left\langle V_{\alpha}, Q_{\delta}\right\rangle$ by (3.2)-(3).

(4) The following holds.

(i) $U_{\alpha+1} \cap V_{\lambda}=1$.

(ii) $G_{\alpha+1} \supseteq Q_{\alpha+2} \supseteq U_{\lambda}^{(2 k-2)} \varsubsetneqq G_{\alpha, \alpha+1}$.

(iii) If $k=1$, then $V_{\alpha+1}=V_{\delta-1}, W_{\delta-1}=U_{\delta} \cap Q_{\alpha+1}$, and $\left[W_{\alpha+2}, U_{\lambda}\right]=1$.

Proof. Since $U_{\alpha+1} \cap V_{\lambda}$ is centralized by $G_{\delta}$ and $G_{\lambda}$, we have $U_{\alpha+1} \cap V_{\lambda}=1$, so (i) holds.

Since $V_{\alpha+2} \subseteq Q_{\lambda}$ and $U_{\lambda}^{(2 k-2)} \subseteq U_{\lambda} \subseteq Q_{\alpha+3}$ by the minimality of $b$, $\left[V_{\alpha+2}, U_{\lambda}^{(2 k-2)}\right] \subseteq V_{\alpha+2} \cap U_{\lambda}^{(2 k-1)} \subseteq U_{\alpha+1} \cap V_{\lambda}=1$ by (i), so $U_{\lambda}^{(2)} \subseteq C_{G_{\alpha+2, \alpha+3}}$ $\left(V_{\alpha+2}\right)=Q_{\alpha+2} \subseteq G_{\alpha+1}$. Let $W=V_{\delta}\left(U_{\lambda}{ }^{(2 k-2)} \cap G_{\alpha, \alpha+1}\right)$. Then we have $\left[W, Q_{\lambda}\right]$ $\subseteq V_{\delta} \subseteq W$ because $\left[U_{\lambda}^{(2 k-2)}, Q_{\lambda}\right]=V_{\lambda} \subseteq V_{\delta}$ by $(3)$.

Suppose first that $k=2$. Since $\left[W, V_{\delta}\right]=1$ by the minimality of $b$,

$$
\left[V_{\alpha}, W\right]=\left[V_{\alpha}, V_{\delta}\right]\left[V_{\alpha} \cap Q_{\delta}, W\right] \subseteq W
$$

by (2) and (2.5)-(6). Thus we have $W \triangleleft\left\langle V_{\alpha}, Q_{\lambda}\right\rangle=G_{\delta}$, and hence $\left[W, G_{\delta}\right]=$ $\left[W,\left\langle Q_{\lambda} G_{\delta}\right\rangle\right] \subseteq V_{\delta}$. Therefore (3.8) shows that $U_{\lambda}{ }^{(2)} \nsubseteq W$ because $\left[U_{\lambda}^{(2)}, O^{2}\left(G_{\lambda}\right)\right]$ $\nsubseteq V_{\lambda}$ by $(2.14)-(1)$.

Suppose next that $k=1$. Then $\left|V_{\alpha}: V_{\alpha+1}\right|=2=\left|V_{\delta}: V_{\delta-1}\right|$, so $V_{\alpha+1}=$ $\left[V_{\alpha}, V_{\delta}\right]=V_{\delta-1}$. Thus we have $\left[V_{\alpha}, W\right] \subseteq V_{\alpha+1}=V_{\delta-1} \subseteq W$, and so $W \triangleleft G_{\delta}$. This shows that $W=W_{\delta} \nsupseteq U_{\lambda}$. Since $W_{\alpha+2} \subseteq U_{\alpha+3} \subseteq Q_{\lambda}$ and $U_{\lambda} \subseteq Q_{\alpha+3}$ by the minimality of $b$, we have $\left[W_{\alpha+2}, U_{\lambda}\right] \subseteq W_{\alpha+2} \cap V_{\lambda} \subseteq U_{\alpha+1} \cap V_{\lambda}=1$ by (i). This proves (ii) and (iii).

Now, we will assume $k=2$, and derive a contradiction.

$$
O^{2}\left(G_{\alpha+1} / Q_{\alpha+1} C_{G_{\alpha+1}}\left(U_{\alpha+1}\right)\right) \cong \mathbf{Z}_{3} \text { or } \mathbf{E}_{3^{2}} .
$$

Proof. By (3) and (4-i), we have $\left[U_{\alpha+1} \cap Q_{\lambda}, U_{\lambda}{ }^{(2)}\right] \subseteq U_{\alpha+1} \cap V_{\lambda}=1$. Since $m\left(G_{\delta-1, \delta} / Q_{\delta}\right) \leqq 2 \geqq m\left(G_{\delta \lambda} / Q_{\lambda}\right)$ by $(2.1)-(6)$,

$$
\begin{aligned}
\left|U_{\alpha+1}: C_{U_{\alpha+1}}\left(U_{\lambda}^{(2)}\right)\right| & \leqq\left|U_{\alpha+1}: U_{\alpha+1} \cap Q_{\lambda}\right| \\
& \leqq\left|U_{\alpha+1}: U_{\alpha+1} \cap Q_{\delta}\right| \cdot\left|U_{\alpha+1} \cap Q_{\delta}: U_{\alpha+1} \cap Q_{\delta} \cap Q_{\lambda}\right| \\
& \leqq 2^{2} \cdot 2^{2}=2^{4} .
\end{aligned}
$$


Since $n\left(G_{\alpha+1}, \quad U_{\alpha+1}\right) \geqq 3$ by (3) and (2.14)-(2), $U_{\lambda}{ }^{(2)}$ induces a factorwise sesquitransvection on $U_{\alpha+1}$ by $(2.11)-(2)$. Hence the assertion follows from (2.11)(1).

(6) A Contradiction.

Proof. Since $V_{\alpha+1}\left[V_{\alpha}, V_{\delta}\right] \neq V_{\alpha+1}$ by $(2.5)-(7)$ and $U_{\lambda}{ }^{(2)}$ centralizes $V_{\alpha+1}\left[V_{\alpha}, V_{\delta}\right]$ by (4-ii) and the minimality of $b$, we have $G_{\alpha+1} \neq\left\langle G_{\alpha, \alpha+1}, U_{\lambda}{ }^{(2)}\right\rangle$. Therefore there exists $x \in G_{\alpha+1}$ such that $U_{\lambda}{ }^{(2)} \subseteq G_{\alpha, \alpha+1}{ }^{x}=G_{\alpha^{z}, \alpha+1}$ and $V_{\alpha^{x}}=V_{\alpha}{ }^{*}=V_{\alpha} \nsubseteq Q_{\delta}$, by (5) and (3.10)-(1). Thus we have $\left(\alpha^{x}, \delta\right) \in \mathcal{C}$, but then this contradicts (4-ii).

We have shown that $k=1$. Thus $n_{*}\left(G_{\alpha}\right)=1$ by $(2.1)-(9)$.

Now, put $\widehat{U}_{\alpha+1}=U_{\alpha+1} / V_{\alpha+1}, C_{\alpha+1}=C_{G_{\alpha+1}}\left(\widehat{U}_{\alpha+1}\right)$, and $\widetilde{G}_{\alpha+1}=G_{\alpha+1} / C_{\alpha+1}$. We have $O^{2}\left(G_{\alpha+1}\right) \varsubsetneqq C_{\alpha+1}$ as $V_{\alpha+1} \subseteq V_{\alpha} \subseteq U_{\alpha+1}$, and $Q_{\alpha+1} \subseteq C_{\alpha+1}$ by (3). Therefore $\widetilde{G}_{\alpha+1}$ is a 2-irreducible solvable group with $O_{2}\left(\widetilde{G}_{\alpha+1}\right)=1$ by $(2.4)-(1)$. If $n_{*}\left(\widetilde{G}_{\alpha+1}\right)=1$, then $O^{2}\left(\widetilde{G}_{\alpha+1}\right)$ is cyclic, and so $n_{*}\left(G_{\alpha+1}\right)=1$ by $(2.1)-(9)$. Since $n_{*}\left(\widetilde{G}_{\alpha+1}\right) \leqq n_{*}\left(G_{\alpha+1}\right) \leqq f\left(G_{\alpha+1}\right) \leqq 2$, we will assume $n_{*}\left(\widetilde{G}_{\alpha+1}\right)=2$, and argue for a contradiction.

(7) $\left|W_{\alpha}\right|=2^{3},\left|U_{\alpha+1}\right|=2^{5},\left[U_{\alpha+1}, U_{\lambda}\right] V_{\alpha+1}=W_{\alpha+2}=C_{U_{\alpha+1} \bmod V_{\alpha+1}}\left(U_{\lambda}\right)$, and $n\left(G_{\alpha}, T_{\alpha} / W_{\alpha}\right) \geqq 2$.

Proof. We will regard $\widehat{U}_{\alpha+1}$ as a faithful $G F(2) \widetilde{G}_{\alpha+1}$-module. By (4-iii) and (2.4)-(1), we have

$$
\left|\widehat{U}_{\alpha+1}: C_{\widehat{U}_{\alpha+1}}\left(\widetilde{U}_{\lambda}\right)\right| \leqq\left|U_{\alpha+1}: W_{\alpha+2}\right|=\left|U_{\lambda}: W_{\delta}\right|=\left|\widetilde{U}_{\lambda}\right|
$$

Since $n_{*}\left(\widetilde{G}_{\alpha+1}\right)=2$ by our hypothesis, we have $O^{2}\left(\widetilde{G}_{\alpha+1}\right) \cong \mathbf{E}_{3^{2}}$ and $\left|\widehat{U}_{\alpha+1}\right| \geqq$ $\left|\left[\widehat{U}_{\alpha+1}, O^{2}\left(\widetilde{G}_{\alpha+1}\right)\right]\right|=2^{4}$ by $(2.10)-(1)$, and hence $\left(G_{\alpha+1}, \widehat{U}_{\alpha+1}\right) \ll\left(O_{4}^{+}(2), G F(2)^{4}\right)$ by (2.5)-(1). Thus (3.8) shows that $\left|W_{\alpha}: V_{\alpha}\right| \leqq 2$, and then $\left|W_{\alpha}: V_{\alpha}\right|=2$ and $\left|U_{\alpha+1}: W_{\alpha+2}\right|=2^{2}$. Consequently, we have $\left|W_{\alpha}\right|=2^{3},\left|U_{\alpha+1}\right|=2^{5}$, and $\left|\widetilde{U}_{\lambda}\right|=$ $2^{2}$. Hence, by $(2.5)-(3)(4)(5), \widehat{W}_{\alpha+2}=\left[\widehat{U}_{\alpha+1}, \widetilde{G}_{\alpha+1, \alpha+2} ; 2\right]$ and $\widetilde{U}_{\lambda}$ is the unique quadratic fours subgroup of $\widetilde{G}_{\alpha+1, \alpha+2}$. Therefore $\left[\widehat{U}_{\alpha+1}, \widetilde{U}_{\lambda}\right]=\widehat{W}_{\alpha+2}=C_{\widehat{U}_{\alpha+1}}\left(\widetilde{U}_{\lambda}\right)$ by (2.5)-(4), and $T_{\alpha}{ }^{(1)} \neq W_{\alpha}$ by (3.9), and then $n\left(G_{\alpha}, T_{\alpha} / W_{\alpha}\right) \geqq 2$ by (2.14)-(2).

(8) $T_{\delta} \subseteq Q_{\alpha+2}$ and $T_{\delta}^{2} \subseteq Q_{\alpha+1}$.

Proof. Note that $T_{\delta} \subseteq Q_{\alpha+3} \cap Q_{\lambda}$ by the minimality of $b$. As $\left[T_{\delta}, U_{\lambda}\right] \subseteq V_{\lambda}$ $\subseteq W_{\delta}$ and $T_{\delta}=\left\langle U_{\lambda}{ }^{G_{\delta}}\right\rangle,\left[T_{\delta}, T_{\delta}\right] \subseteq W_{\delta}$, and so $T_{\delta}{ }^{2} \subseteq W_{\delta} \subseteq Q_{\alpha+1}$. By (4-iii), we have 
$\left[V_{\alpha}, T_{\delta} \cap Q_{\alpha+1}\right] \subseteq V_{\alpha+1}=V_{\delta-1} \subseteq W_{\delta}$. Therefore

$$
\begin{aligned}
\left|T_{\delta} / W_{\delta}: C_{T_{\delta} / W_{\delta}}\left(V_{\alpha}\right)\right| & \leqq\left|T_{\delta}: T_{\delta} \cap Q_{\alpha+1}\right| \\
& \leqq\left|T_{\delta}: T_{\delta} \cap Q_{\alpha+2}\right| \cdot\left|T_{\delta} \cap Q_{\alpha+2}: T_{\delta} \cap Q_{\alpha+2} \cap Q_{\alpha+1}\right| \\
& \leqq 2 \cdot 2^{2}=2^{3}
\end{aligned}
$$

because $m\left(G_{\alpha+2, \alpha+3} / Q_{\alpha+2}\right) \leqq 1$ and $m\left(G_{\alpha+1, \alpha+2} / Q_{\alpha+1}\right) \leqq 2$. Thus $V_{\alpha}$ induces a factorwise sesquitransvection on $T_{\delta} / W_{\delta}$ by (7) and (2.11)-(2). Since $G_{\delta}$ is a $\{2,3\}$ group with cyclic Sylow 3 -subgroups, we conclude that $O^{2}\left(G_{\delta} / Q_{\delta} C_{G_{\delta}}\left(T_{\delta} / W_{\delta}\right)\right) \cong$ $\mathbf{Z}_{3}$ by (2.11)-(1), and hence $T_{\delta}=\left\langle U_{\delta-1}, U_{\lambda}, U_{\lambda}{ }^{x}\right\rangle$ for some $x \in V_{\alpha}-Q_{\delta}$. This shows that $T_{\delta} \subseteq Q_{\alpha+2}$.

(9) A contradiction.

Proof. Since $\left|U_{\alpha+1}: U_{\alpha+1} \cap Q_{\delta}\right|=2$, we have $W_{\alpha+2} \subset U_{\alpha+1} \cap Q_{\delta}$, so $V_{\alpha+1} \subset\left[U_{\alpha+1} \cap Q_{\delta}, U_{\lambda}\right] V_{\alpha+1} \subseteq W_{\alpha+2} \cap U_{\lambda} \subseteq W_{\alpha+2} \cap W_{\delta}$. Hence

$$
\left|W_{\alpha+2} W_{\delta}: W_{\delta}\right|=\left|W_{\alpha+2}: W_{\alpha+2} \cap W_{\delta}\right| \leqq 2 .
$$

Since $\left|\widetilde{U}_{\lambda}\right|=\left|U_{\lambda}: W_{\delta}\right|=2^{2}$ by $(7)$ and $\widetilde{T}_{\delta}$ is an elementary abelian subgroup of $\widetilde{G}_{\alpha+1, \alpha+2}$ containing $\widetilde{U}_{\lambda}$ by $(8)$, we have $\widetilde{T}_{\delta}=\widetilde{U}_{\lambda}$, and so

$$
\left[U_{\alpha+1}, T_{\delta}\right] \subseteq\left[U_{\alpha+1}, U_{\lambda}\right] V_{\alpha+1}=W_{\alpha+2} \subseteq W_{\alpha+2} W_{\delta}
$$

by (7). Therefore we have $n\left(G_{\alpha}, T_{\alpha} / W_{\alpha}\right) \leqq 1$ by (2.11)-(2), which contradicts (7). This completes the proof of $(5.1)$.

\section{$\S 6 . \quad$ Odd case}

In the remainder of this paper, we will assume that $b$ is odd and that $b \geqq 3$. The main aim of this section is to prove that $n_{*}\left(G_{\xi}\right)=1$ for some (and hence all) $\xi \in \mathcal{A}$.

Define

$$
\begin{aligned}
U_{\eta} & =\left\langle V_{\xi} \mid \xi \in \Delta(\eta)\right\rangle \quad \text { for } \quad \eta \in \mathcal{B}, \quad \text { and } \\
\mathcal{C}^{\prime} & =\left\{(\beta, \delta) \mid \beta, \delta \in \mathcal{B}, U_{\beta} \nsubseteq Q_{\delta}, \quad \text { and } \quad d(\beta, \delta)=b-1\right\} .
\end{aligned}
$$

Suppose $(\alpha, \delta) \in \mathcal{C}$, and that $(\alpha, \beta, \ldots, \delta)$ is a path of length $b$ from $\alpha$ through $\delta$. Then we have $\beta \in \mathcal{B} \ni \delta$ by (4.5) and (4.6) as in Section 4, and, moreover, $U_{\beta} \not Q_{\delta}$ and $d(\beta, \delta)=b-1$ by the definition of $\mathcal{C}$. This shows that $\mathcal{C}^{\prime} \neq \phi$.

Lemma 6.1 Let $\beta, \delta \in \mathcal{B}$, and suppose $d(\beta, \delta)=b-1$. Then

$$
\left|V_{\alpha}: V_{\alpha} \cap Q_{\delta}\right| \leqq 2 \text { for all } \alpha \in \Delta(\beta) \text {. }
$$


Proof. We will assume that $\left|V_{\alpha}: V_{\alpha} \cap Q_{\delta}\right| \geqq 2^{2}$ for some $\alpha \in \Delta(\beta)$, and argue for a contradiction.

Let $(\beta, \beta+1, \ldots, \delta-1, \delta)$ be a path of length $b-1$ from $\beta$ through $\delta$. Then we have $U_{\beta} \subseteq Q_{\delta-1}$ and $U_{\delta} \subseteq Q_{\beta+1}$ by the minimality of $b$. Since $m\left(G_{\delta-1, \delta} / Q_{\delta}\right) \leqq 2$ by $(2.1)-(6)$, we have $\Omega_{1}\left(Z\left(G_{\delta-1, \delta} / Q_{\delta}\right)\right) \subset V_{\alpha} Q_{\delta} / Q_{\delta}$. Let $\widehat{U}_{\delta}=U_{\delta} / V_{\delta}$. Then $n\left(G_{\delta}, \widehat{U}_{\delta}\right) \geqq 1$ and

$$
\begin{aligned}
\left|\widehat{U}_{\delta}: C_{\widehat{U}_{\delta}}\left(V_{\alpha}\right)\right| & \leqq\left|U_{\delta}: U_{\delta} \cap Q_{\alpha}\right| \\
& \leqq\left|U_{\delta}: U_{\delta} \cap Q_{\beta}\right| \cdot\left|U_{\delta} \cap Q_{\beta}: U_{\delta} \cap Q_{\beta} \cap Q_{\alpha}\right|
\end{aligned}
$$

Suppose first that $n_{*}\left(G_{\delta-1}\right)=1$. Then $G_{\alpha} \beta / Q_{\alpha}$ is cyclic by $(2.1)-(7)$, and so $\left|\widehat{U}_{\delta}: C_{\widehat{U}_{\delta}}\left(V_{\alpha}\right)\right| \leqq 2^{2} \cdot 2=2^{3}$. Hence $n\left(G_{\delta}, \widehat{U}_{\delta}\right)=1$ and $V_{\alpha}$ induces a factorwise semioffending action on $\widehat{U}_{\delta}$ by $(2.11)-(3)$. Thus $\left(G_{\delta}, \widehat{U}_{\delta}\right) \ll\left(O_{4}^{+}(2), G F(2)^{4}\right)$ by (2.11)-(1) and (2.5)-(2), and so $\left|V_{\delta-1, \delta}: V_{\delta}\right| \leqq 2$ by (2.15). This shows that $\left|V_{\alpha}: V_{\alpha} \cap Q_{\delta}\right| \leqq\left|V_{\alpha}: V_{\beta}\right| \leqq 2$, a contradiction.

Suppose next that $n_{*}\left(G_{\delta-1}\right)=2$. Put $\bar{G}_{\delta-1}=G_{\delta-1} / C_{G_{\delta-1}}\left(V_{\delta-1}\right)$ and regard $V_{\delta-1}$ as a faithful $G F(2) \bar{G}_{\delta-1}$-module. Since $n_{*}\left(\bar{G}_{\delta-1}\right)=2$ by $(2.1)-(9)$, we have $\left[V_{\delta-1}, Q_{\delta} ; 2\right]=\left[V_{\delta-1}, G_{\delta-1, \delta} ; 2\right] \neq 1$ by $(2.12)-(1)$. Hence $\left[U_{\delta}, Q_{\delta}\right] \varsubsetneqq \cap V_{\delta}^{G_{\delta-1}}=$ $V_{\delta-1}$, and so $n\left(G_{\delta}, \widehat{U}_{\delta}\right) \geqq 2$. Thus, by $(2.11)-(3), n\left(G_{\delta}, \widehat{U}_{\delta}\right)=2$ and $V_{\alpha}$ induces a factorwise semioffending action on $\widehat{U}_{\delta}$ because $\left|\widehat{U}_{\delta}: C_{\widehat{U}_{\delta}}\left(V_{\alpha}\right)\right| \leqq 2^{2} \cdot 2^{2}=2^{4}$. Hence we have $\left|V_{\delta-1, \delta}: V_{\delta}\right| \leqq 2^{2}$ as before by (2.11)-(1), (2.5)-(2), and (2.15). But then $O^{2}\left(\bar{G}_{\delta-1}\right)$ is cyclic and nonidentity by $(2.12)-(2)$, and so $n_{*}\left(G_{\delta-1}\right)=1$ by (2.1)-(9), a contradiction.

Theorem 6.2 Let $\xi \in \mathcal{A}$ and $\eta \in \mathcal{B}$. If $b$ is odd and $b \geqq 3$, then $n_{*}\left(G_{\xi}\right)=1$. More precisely,

$$
G_{\xi} / C_{G_{\xi}}\left(V_{\xi}\right) \cong \mathbf{D}_{6} \quad \text { or } \quad \mathbf{D}_{10}, \quad \text { and } \quad\left|V_{\xi}\right|=\left|V_{\eta}\right|^{2}=2^{2} \quad \text { or } \quad 2^{4} .
$$

Proof. Let $(\beta, \delta) \in \mathcal{C}^{\prime}$. Take $\alpha \in \Delta(\beta)$ and $\lambda \in \Delta(\delta)$ so that $G_{\delta}=\left\langle V_{\alpha}, G_{\delta \lambda}\right\rangle=$ $\left\langle V_{\alpha}, Q_{\lambda}\right\rangle$. Note that $V_{\delta}=V_{\lambda} \cap Q_{\alpha}$. We may assume $\xi=\lambda$ and $\eta=\delta$.

Suppose that $\left|V_{\lambda}: V_{\delta}\right| \geqq 2^{3}$. Then we have $\left|V_{\lambda}: V_{\lambda} \cap Q_{\beta}\right|=2$, $\left|V_{\lambda} \cap Q_{\beta}: V_{\lambda} \cap Q_{\beta} \cap Q_{\alpha}\right|=\left|V_{\lambda} \cap Q_{\beta}: V_{\delta}\right|=2^{2}$, and $\left|V_{\alpha}: C_{V_{\alpha}}\left(V_{\lambda} \cap Q_{\beta}\right)\right| \leqq \mid V_{\alpha}: V_{\alpha} \cap$ $Q_{\lambda} \mid \leqq 2^{3}$ by $(6.1)$. Thus $\Omega_{1}\left(Z\left(G_{\alpha \beta} / Q_{\alpha}\right)\right) \subset\left(V_{\lambda} \cap Q_{\beta}\right) Q_{\alpha} / Q_{\alpha}$, so $O^{2}\left(G_{\alpha} / C_{G_{\alpha}}\left(V_{\alpha}\right)\right)$ $\cong \mathbf{E}_{3^{2}}$ and $\left|V_{\alpha}\right|=2^{4}$ by (2.10)-(2) and (3.6). Therefore we have $\left[U_{\beta}, Q_{\beta} ; 3\right]=V_{\beta}=$ $\cap V_{\alpha}^{G_{\beta}}$ similarly as in (3) of (5.1), and hence $n\left(G_{\beta}, U_{\beta} / V_{\beta}\right) \geqq 3$ by (2.14)-(2). Since $\left|U_{\beta}: C_{U_{\beta}}\left(V_{\lambda}\right)\right| \leqq\left|U_{\beta}: U_{\beta} \cap Q_{\lambda}\right| \leqq 2^{4}, O^{2}\left(G_{\beta} / Q_{\beta} C_{G_{\beta}}\left(U_{\beta} / V_{\beta}\right)\right) \cong \mathbf{Z}_{3}$ or $\mathbf{E}_{3^{2}}$ similarly as in (5) of (5.1). Since $V_{\beta} \neq\left[V_{\alpha}, V_{\lambda} \cap Q_{\beta}\right] \subseteq V_{\alpha} \cap U_{\delta}$, we have $G_{\beta} \neq\left\langle Q_{\alpha}, U_{\delta}\right\rangle$, so $G_{\beta} \neq\left\langle G_{\alpha \beta}, U_{\delta}\right\rangle$ by (3.2)-(3). Thus there exists an element $x \in G_{\beta}$ such that $U_{\delta} \subseteq G_{\alpha^{x}, \beta}$ and $V_{\alpha^{x}}=V_{\alpha}$ by (3.10)-(1). Therefore $V_{\delta}=V_{\lambda} \cap Q_{\alpha^{x}}$, and so 
$\left|V_{\lambda}: V_{\delta}\right|=\left|V_{\lambda} Q_{\alpha^{z}}: Q_{\alpha^{z}}\right| \leqq 2^{2}$, a contradiction. This shows that $\left|V_{\lambda}: V_{\delta}\right| \leqq 2^{2}$. Let $\bar{G}_{\lambda}=G_{\lambda} / C_{G_{\lambda}}\left(V_{\lambda}\right)$, and regard $V_{\lambda}$ as a faithful $G F(2) \bar{G}_{\lambda}$-module. Then we have $\left|V_{\lambda}: C_{V_{\lambda}}\left(\bar{G}_{\delta \lambda}\right)\right| \leqq 2^{2}$, and hence the assertions follow from (3.6), (2.12)-(2), and $(2.1)-(7)$.

Corollary 6.3 Let $\eta \in \mathcal{B}$. Then $U_{\eta} / V_{\eta}$ is elementary abelian, $O^{2}\left(G_{\eta}\right) \nsubseteq$ $C_{G_{\eta}}\left(U_{\eta} / V_{\eta}\right)$, and $\left[U_{\eta}, Q_{\eta}\right]=V_{\eta}$.

Proof. Let $\eta \in \mathcal{B}$ and $\xi \in \Delta(\eta)$. Note that $U_{\eta}$ is elementary abelian as $b \geqq 3$. We have $O^{2}\left(G_{\eta}\right) \varsubsetneqq C_{G_{\eta}}\left(U_{\eta} / V_{\eta}\right)$ because $V_{\eta} \subseteq V_{\xi} \subseteq U_{\eta}$. Since $G_{\xi \eta}=Q_{\xi} Q_{\eta}$ by $(3.2)-(1)$, we have $\left[V_{\xi}, Q_{\eta}\right]=\left[V_{\xi}, G_{\xi \eta}\right]=V_{\eta}$ by $(6.2)$, so $\left[U_{\eta}, Q_{\eta}\right]=\left[\left\langle V_{\xi} G_{\eta}\right\rangle, Q_{\eta}\right]=$ $\left\langle\left[V_{\xi}, Q_{\eta}\right]^{G_{\eta}}\right\rangle=V_{\eta}$.

Corollary 6.4 Let $(\beta, \delta) \in \mathcal{C}^{\prime}$. Then

$$
\left|V_{\beta} V_{\delta}: V_{\beta}\right|=\left|V_{\beta} V_{\delta}: V_{\delta}\right| \leqq 2
$$

In particular, $V_{\beta} \cap V_{\delta} \neq 1$ if $\left|V_{\xi}\right|=2^{4}$ for $\xi \in \mathcal{A}$.

Proof. By (6.2), we may assume $\left|V_{\beta}\right|=\left|V_{\delta}\right|=2^{2}$, and hence it suffices to prove that $V_{\beta} \cap V_{\delta} \neq 1$. Take $\alpha \in \Delta(\beta)$ and $\lambda \in \Delta(\delta)$ so that $G_{\delta}=\left\langle V_{\alpha}, G_{\delta \lambda}\right\rangle=$ $\left\langle V_{\alpha}, Q_{\lambda}\right\rangle$. Then $V_{\delta}=V_{\lambda} \cap Q_{\alpha}$. Since $\left|V_{\alpha}: V_{\beta}\right|=\left|V_{\lambda}: V_{\delta}\right|=2^{2}$, we have $V_{\beta} \subset V_{\alpha} \cap Q_{\delta}$ and $V_{\delta} \subset V_{\lambda} \cap Q_{\beta}$ by (6.1). Hence $1 \neq\left[V_{\alpha} \cap Q_{\delta}, V_{\lambda} \cap Q_{\beta}\right] \subseteq V_{\beta} \cap V_{\delta}$.

Lemma 6.5 One of the following holds.

(1) If $(\beta, \delta) \in \mathcal{C}^{\prime}$, then

(i) $\left|U_{\beta}: U_{\beta} \cap Q_{\delta}\right|=\left|U_{\delta}: U_{\delta} \cap Q_{\beta}\right|=2$,

(ii) $U_{\beta}$ (resp. $\left.U_{\delta}\right)$ induces a quasitransvection but no transvection on $U_{\delta} / V_{\delta}\left(\right.$ resp. $\left.U_{\beta} / V_{\beta}\right)$,

(iii) $C_{U_{\delta} \bmod V_{\delta}}\left(U_{\beta}\right) \subseteq Q_{\beta}$ and $C_{U_{\beta} \bmod V_{\beta}}\left(U_{\delta}\right) \subseteq Q_{\delta}$,

(iv) $V_{\beta} V_{\delta} \subseteq\left[U_{\beta}, U_{\delta}\right] \subseteq U_{\beta} \cap U_{\delta}$, and

(v) $\quad\left|V_{\beta} V_{\delta}: V_{\beta}\right|=\left|V_{\beta} V_{\delta}: V_{\delta}\right|=\left|\left[U_{\beta}, U_{\delta}\right]: V_{\beta} V_{\delta}\right|=2$.

(2) Let $\eta \in \mathcal{B}$, and put $\widetilde{G}_{\eta}=G_{\eta} / C_{G_{\eta}}\left(U_{\eta} / V_{\eta}\right)$ and $\widehat{U}_{\eta}=\left[U_{\eta} / V_{\eta}, O^{2}\left(\widetilde{G}_{\eta}\right)\right]$. Then

$$
O^{2}\left(\widetilde{G}_{\eta}\right) \cong \mathbf{E}_{3^{k}} \quad \text { and } \quad\left|\widehat{U}_{\eta}\right|=2^{2 k}
$$

where $k=1$ or 2 . Moreover, $m_{2}\left(\widetilde{G}_{\eta}\right) \geqq 2$ if $k=2$.

Proof. Suppose (2) does not hold. Let $(\beta, \delta) \in \mathcal{C}^{\prime}$, and let $(\beta, \beta+1, \ldots, \delta)$ be a path of length $b$ from $\beta$ through $\delta$.

We will first prove (i) of (1). Assume $U_{\delta} \subseteq Q_{\beta}$. Since $\left[U_{\beta}, U_{\delta}\right] \subseteq V_{\beta} \subseteq V_{\beta} V_{\delta}$ and $\left|V_{\beta} V_{\delta}: V_{\delta}\right| \leqq 2$ by (6.3) and (6.4), $U_{\beta}$ induces a transvection on $U_{\delta} / V_{\delta}$. Thus (2) holds for $\delta$ by (2.10)-(1), a contradiction. Now, by symmetry, we may assume $\mid U_{\delta}$ : $U_{\delta} \cap Q_{\beta}|=| U_{\delta} Q_{\beta}: Q_{\beta} \mid=2^{2}$. Define $\widetilde{G}_{\beta}$ and $\widehat{U}_{\beta}$ as in $(2)$. Note that $m_{2}\left(\widetilde{G}_{\beta}\right) \geqq 2$, 
and so $\Omega_{1}\left(Z\left(\widetilde{G}_{\beta, \beta+1}\right)\right) \subset \widetilde{U}_{\delta}$ and $n_{*}\left(G_{\beta}\right)=2$ by $(2.1)-(5)(7)$. Take $z \in U_{\delta}-Q_{\beta}$ so that $\tilde{z} \in \Omega_{1}\left(Z\left(\widetilde{G}_{\beta, \beta+1}\right)\right)$. Then $\left[U_{\beta} \cap Q_{\delta}, z\right] \subseteq\left[U_{\beta} \cap Q_{\delta}, U_{\delta}\right] \subseteq V_{\delta} \subseteq V_{\beta} V_{\delta}$, and so $\left|\widehat{U}_{\beta}: C_{\widehat{U}_{\beta}}(z)\right| \leqq\left|\widehat{U}_{\beta}: C_{\widehat{U}_{\beta}}(z)\right| \leqq 2^{3}$. If $n\left(G_{\beta}, \widehat{U}_{\beta}\right) \geqq 2$, then $z$ induces a factorwise sesquitransvection on $\widehat{U}_{\beta}$ by $(2.11)-(2)$, and so $n_{*}\left(G_{\beta}\right)=1$ by $(2.11)-(1)$ and (2.1)-(9), a contradiction. If $n\left(G_{\beta}, \widehat{U}_{\beta}\right)=1$, then $\widehat{U}_{\beta}$ is irreducible and faithful as a $G F(2) \widetilde{G}_{\beta^{-}}$-module by $(2.4)-(2)(3)$, and hence either $O^{2}\left(\widetilde{G}_{\beta}\right) \cong \mathbf{E}_{3^{2}}$ and $\left|\widehat{U}_{\beta}\right|=2^{4}$, or $O^{2}\left(\widetilde{G}_{\beta}\right) \cong E s p_{27}$ and $\left|\widehat{U}_{\beta}\right|=2^{6}$. However, since $\left[U_{\beta}, z, U_{\delta}\right] \subseteq\left[U_{\beta}, U_{\delta}, U_{\delta}\right]=1$, we have $O^{2}\left(\widetilde{G}_{\beta}\right) \not E s p_{27}$ by (2.7)-(6), and hence (2) holds for $\beta$. This proves (i) of (1).

Take $\alpha \in \Delta(\beta)$ and $\lambda \in \Delta(\delta)$ so that $V_{\alpha} \nsubseteq Q_{\delta}$ and $V_{\lambda} \nsubseteq Q_{\beta}$. Since $U_{\beta}=$ $V_{\alpha}\left(U_{\beta} \cap Q_{\delta}\right)$ by (i) and $\left|U_{\delta}: U_{\delta} \cap Q_{\alpha}\right| \leqq 2^{2}, U_{\beta}$ induces a quasitransvection on $U_{\delta} / V_{\delta}$, and, by symmetry, $U_{\delta}$ also induces a quasitransvection on $U_{\beta} / V_{\beta}$. Moreover, neither of them is a transvection because (2) does not hold. Thus we have $U_{\delta} \cap Q_{\beta} \nsubseteq Q_{\alpha}$ and $U_{\beta} \cap Q_{\delta} \nsubseteq Q_{\lambda}$, and hence $C_{U_{\delta} \bmod V_{\delta}}\left(U_{\beta}\right)=U_{\delta} \cap Q_{\beta} \cap Q_{\alpha} \subset U_{\delta} \cap Q_{\beta}$ and $C_{U_{\beta} \bmod V_{\beta}}\left(U_{\delta}\right)=U_{\beta} \cap Q_{\delta} \cap Q_{\lambda} \subset U_{\beta} \cap Q_{\delta}$. This shows that

$$
V_{\beta} V_{\delta}=\left[U_{\beta}, U_{\delta} \cap Q_{\beta}\right]\left[U_{\beta} \cap Q_{\delta}, U_{\delta}\right] \subseteq\left[U_{\beta}, U_{\delta}\right] \subseteq U_{\beta} \cap U_{\delta},
$$

and so $\left|V_{\beta} V_{\delta}: V_{\beta}\right|=\left|V_{\beta} V_{\delta}: V_{\delta}\right|=\left|\left[U_{\beta}, U_{\delta}\right]: V_{\beta} V_{\delta}\right|=2$ by (6.4). This proves (ii) $-(\mathrm{v})$ of (1).

\section{$\S 7 . \quad$ Odd case with $b=3$}

In this section, we will consider the case where $b=3$.

Theorem 7.1 Let $\eta \in \mathcal{B}$. If $b=3$, then $n_{*}\left(G_{\eta}\right)=1$.

Proof. Suppose false. Let $(\beta, \delta) \in \mathcal{C}^{\prime}$ and $\gamma \in \Delta(\beta) \cap \Delta(\delta)$.

(1) $V_{\beta} \neq V_{\delta}$

Proof. Suppose false. Then we have $\left[U_{\beta}, U_{\delta} \cap Q_{\beta}\right] \subseteq V_{\beta}=V_{\delta}$ by (6.3), so $U_{\delta} \nsubseteq Q_{\beta}$ because $U_{\beta} \nsubseteq Q_{\delta}=C_{G_{\delta-1, \delta}}\left(U_{\delta} / V_{\delta}\right)$ by (6.3) and (2.4)-(1). Thus, by symmetry, we may assume $\left|U_{\beta}: U_{\beta} \cap Q_{\delta}\right| \leqq\left|U_{\delta}: U_{\delta} \cap Q_{\beta}\right|$. Then $U_{\delta}$ induces an offending action on $U_{\beta} / V_{\beta}$. Note that $U_{\delta} Q_{\beta} \supseteqq Z_{\beta \gamma}$ because $G_{\beta \gamma}=Q_{\beta} Q_{\gamma}$ and $Q_{\gamma} \triangleright U_{\delta}$. Since $n_{*}\left(G_{\beta} / C_{G_{\beta}}\left(U_{\beta} / V_{\beta}\right)\right)>1$, we have $\left|U_{\delta}: U_{\delta} \cap Q_{\beta}\right|=2^{2}$ and $O^{2}\left(G_{\beta} / C_{G_{\beta}}\left(U_{\beta} / V_{\beta}\right)\right) \cong \mathbf{E}_{3^{2}}$ by $(2.10)-(1)$, and so $G_{\beta \gamma} / Q_{\beta} \cong \mathbf{D}_{8}$ and $\mid U_{\beta}: U_{\beta} \cap$ $Q_{\delta} \mid=2^{2}$ by $(2.5)-(1)(5)$. Since $\left[U_{\beta}, Q_{\beta} \cap Q_{\gamma}\right] \subseteq V_{\beta} \subseteq Q_{\delta}$ and $Q_{\gamma} /\left(Q_{\gamma} \cap Q_{\delta}\right) \cong$ $G_{\gamma \delta} / Q_{\delta} \cong \mathbf{D}_{8}$, we have $Q_{\gamma} \neq\left(Q_{\beta} \cap Q_{\gamma}\right)\left(Q_{\gamma} \cap Q_{\delta}\right)$, so $\left(Q_{\beta} \cap Q_{\gamma}\right)\left(Q_{\gamma} \cap Q_{\delta}\right)=$ $U_{\beta}\left(Q_{\gamma} \cap Q_{\delta}\right)$. Hence $Q_{\beta} \cap Q_{\gamma}=U_{\beta}\left(Q_{\beta} \cap Q_{\gamma} \cap Q_{\delta}\right)$, and so $\left[Q_{\beta} \cap Q_{\gamma}, U_{\delta}\right] \subseteq U_{\beta}$. This shows that $U_{\delta}$ induces a factorwise transvection on $Q_{\beta} / U_{\beta}$, which contradicts (2.11)-(1).

$$
G_{\gamma}=\left\langle G_{\beta \gamma}, G_{\gamma \delta}\right\rangle=\left\langle Q_{\beta}, Q_{\delta}\right\rangle \text { and } Q_{\gamma}=\left(Q_{\beta} \cap Q_{\gamma}\right)\left(Q_{\gamma} \cap Q_{\delta}\right)
$$


Proof. Since $O^{2}\left(G_{\gamma} / C_{G_{\gamma}}\left(V_{\gamma}\right)\right)$ has prime order by $(6.2)$, we have $G_{\gamma}=$ $\left\langle G_{\beta \gamma}, G_{\gamma \delta}\right\rangle$ by (1) and (3.10)-(2). The remaining assertions follow from (3.2)-(3).

Notation. Let $\langle\xi, \eta\rangle \in E(\Gamma)$.

$$
\begin{aligned}
& Z_{\xi \eta}=\Omega_{1}\left(Z\left(G_{\xi \eta} \bmod Q_{\eta}\right)\right), \\
& W_{\xi}=\left\langle\left[U_{\eta}, Z_{\xi \eta}\right]^{G \xi}\right\rangle, \\
& C_{\xi}=C_{G_{\xi}}\left(W_{\xi}\right), \\
& T_{\xi}=\left\langle U_{\eta}^{G_{\xi}}\right\rangle, \text { and } \\
& T_{\xi}^{(1)}=\left[T_{\xi}, Q_{\xi}\right] .
\end{aligned}
$$

(3) $\quad U_{\beta} Q_{\delta}=Z_{\gamma \delta}, U_{\delta} Q_{\beta}=Z_{\beta \gamma}$, and $U_{\beta}\left(\right.$ resp. $\left.U_{\delta}\right)$ induces a quasitransvection on $U_{\delta} / V_{\delta}$ (resp. $U_{\beta} / V_{\beta}$ ), but no factorwise sesquitransvection on $Q_{\delta}$ (resp. $\left.Q_{\beta}\right)$.

Proof. Since $\left[U_{\beta}, Q_{\beta} \cap Q_{\gamma}\right] \subseteq V_{\beta} \subseteq Q_{\delta} \nsupseteq U_{\beta}$, we have $U_{\beta} Q_{\delta}=Z_{\gamma \delta}$, so $U_{\beta}$ induces no factorwise sesquitransvection on $Q_{\delta}$ by $(2.11)-(1)$ because $n_{*}\left(G_{\delta}\right)>$ 1. If $U_{\delta} \subseteq Q_{\beta}$, then $\left[U_{\beta}, U_{\delta}\right] \subseteq V_{\beta}$, so $U_{\beta}$ induces a transvection on $U_{\delta} / V_{\delta}$, a contradiction. Hence $U_{\delta} \nsubseteq Q_{\beta}$, so, by symmetry, $U_{\delta} Q_{\beta}=Z_{\beta \gamma}$ and $U_{\delta}$ induces no factorwise sesquitransvection on $Q_{\beta}$. Since $\left|U_{\beta}: U_{\beta} \cap Q_{\delta}\right|=\left|U_{\delta}: U_{\delta} \cap Q_{\beta}\right|=2$, we conclude that $U_{\beta}$ (resp. $U_{\delta}$ ) induces a quasitransvection on $U_{\delta} / V_{\delta}$ (resp. $U_{\beta} / V_{\beta}$ ) as in the proof of (6.5).

(4) Let $\langle\xi, \eta\rangle \in E(\Gamma)$. Then the following holds.

(i) $\quad G_{\xi} / C_{G_{\xi}}\left(V_{\xi}\right) \cong \mathbf{D}_{6},\left|V_{\xi}\right|=2^{2},\left|W_{\xi}: V_{\xi}\right|=2$, and $\left|Q_{\xi}: C_{\xi}\right|=2^{2}$.

(ii) Either

$$
\begin{aligned}
& O^{2}\left(G_{\eta} / C_{G_{\eta}}\left(U_{\eta} / V_{\eta}\right)\right) \cong \mathbf{E}_{3^{2}} \text { and }\left|U_{\eta} / V_{\eta}\right|=2^{4} \text {, or } \\
& O^{2}\left(G_{\eta} / C_{G_{\eta}}\left(U_{\eta} / V_{\eta}\right)\right) \cong E s p_{27} \text { and }\left|U_{\eta} / V_{\eta}\right|=2^{6} .
\end{aligned}
$$

(iii) $W_{\xi}=\left[U_{\eta}, Z_{\xi \eta}\right] \subseteq T_{\xi}, C_{G_{\xi \eta}}\left(W_{\xi} / V_{\eta}\right)=C_{\xi} Q_{\eta},\left|G_{\xi \eta}: C_{\xi} Q_{\eta}\right|=2$, and $\left|Q_{\eta}: C_{\xi} \cap Q_{\eta}\right|=2^{2}$.

Proof. We may assume $\xi=\gamma$ and $\eta=\beta$. Since $V_{\beta} \cap V_{\delta}$ is centalized both by $G_{\beta}$ and by $G_{\gamma}$ by $(2)$, we have $V_{\beta} \cap V_{\delta}=1$, and so $G_{\gamma} / C_{G_{\gamma}}\left(V_{\gamma}\right) \cong \mathbf{D}_{6}$ and $\left|V_{\gamma}\right|=2^{2}$ by $(6.2)$ and $(6.4)$.

By (3), we have $V_{\gamma}=V_{\beta} V_{\delta} \subseteq\left[U_{\beta}, U_{\delta}\right]$ and $\left|\left[U_{\beta}, U_{\delta}\right]: V_{\gamma}\right|=2$ as in the proof of (6.5). Thus $\left[U_{\beta}, Z_{\beta \gamma}\right]=\left[U_{\beta}, U_{\delta}\right] \triangleleft\left\langle Q_{\beta}, Q_{\delta}\right\rangle=G_{\gamma}$ by (2). Therefore $W_{\gamma}=$ $\left[U_{\beta}, Z_{\beta \gamma}\right] \subseteq U_{\beta} \subseteq Q_{\gamma}$ and $\left.\mid W_{\gamma}: V_{\gamma}\right]=2$, and hence $T_{\gamma} \subseteq C_{Q_{\gamma}}\left(W_{\gamma}\right)=C_{\gamma}$ and $\left|Q_{\gamma}: C_{\gamma}\right| \leqq 2^{2}$.

Let $E_{\gamma \delta}=C_{G_{\gamma \delta}}\left(W_{\gamma} / V_{\delta}\right)$. Then $C_{\gamma} \subseteq E_{\beta \gamma} \cap E_{\gamma \delta}$, and $Q_{\gamma}=\left(Q_{\beta} \cap Q_{\gamma}\right)$. $\left(Q_{\gamma} \cap Q_{\delta}\right)=\left(E_{\beta \gamma} \cap Q_{\gamma}\right)\left(Q_{\gamma} \cap E_{\gamma \delta}\right)$ by (2). Note that $V_{\gamma}=\Omega_{1}\left(Z\left(Q_{\gamma}\right)\right)$ because 
$V_{\beta}=C_{\Omega_{1}\left(Z\left(Q_{\gamma}\right)\right)}\left(G_{\beta \gamma}\right)$. Thus $Q_{\gamma} \nsubseteq E_{\gamma \delta}$, and so we have $\left|Q_{\gamma}: C_{\gamma}\right|=2^{2}$ and $C_{\gamma}=$ $E_{\beta \gamma} \cap E_{\gamma \delta}=\left(Q_{\beta} \cap C_{\gamma}\right)\left(C_{\gamma} \cap Q_{\delta}\right)$. Therefore $E_{\gamma \delta}=C_{\gamma} Q_{\delta}$ and $\left|Q_{\delta}: C_{\gamma} \cap Q_{\delta}\right|=2^{2}$. Hence (i) and (iii) hold.

Let $\widetilde{G}_{\beta}=G_{\beta} / C_{G_{\beta}}\left(U_{\beta} / V_{\beta}\right)$. Then

$$
U_{\beta} / V_{\beta}=\left[U_{\beta} / V_{\beta}, O^{2}\left(\widetilde{G}_{\beta}\right)\right] \times C_{U_{\beta} / V_{\beta}}\left(O^{2}\left(\widetilde{G}_{\beta}\right)\right)
$$

by (2.4)-(3) and (6.3). Note that $U_{\delta}$ induces a quasitransvection but no factorwise transvection on $\left[U_{\beta} / V_{\beta}, O^{2}\left(\widetilde{G}_{\beta}\right)\right]$ by $(3)$. Hence $\left[U_{\beta} / V_{\beta}, O^{2}\left(\widetilde{G}_{\beta}\right)\right]$ is irreducible as a $G F(2) \widetilde{G}_{\beta}$-module and $\left[U_{\beta} / V_{\beta}, U_{\delta}\right] \subseteq\left[U_{\beta} / V_{\beta}, O^{2}\left(\widetilde{G}_{\beta}\right)\right]$. Thus $V_{\gamma} \subseteq\left[U_{\beta}, U_{\delta}\right] \subseteq$ $V_{\beta}\left[U_{\beta}, O^{2}\left(\widetilde{G}_{\beta}\right)\right]$, so $U_{\beta}=\left\langle V_{\gamma} G_{\beta}\right\rangle=V_{\gamma} V_{\beta}\left[U_{\beta}, O^{2}\left(\widetilde{G}_{\beta}\right)\right]=V_{\beta}\left[U_{\beta}, O^{2}\left(G_{\beta}\right)\right]$. Since $U_{\beta} / V_{\beta}$ is a faithful $G F(2) \widetilde{G}_{\beta^{-}}$-module by $(2.4)-(2)$, we have either $O^{2}\left(\widetilde{G}_{\beta}\right) \cong \mathbf{E}_{3^{2}}$ and $\left|U_{\beta} / V_{\beta}\right|=2^{4}$, or $O^{2}\left(\widetilde{G}_{\beta}\right) \cong E s p_{27}$ and $\left|U_{\beta} / V_{\beta}\right|=2^{6}$ by (2.9). Hence (ii) holds.

Now, we divide the proof into two parts.

Case I. $O^{2}\left(G_{\eta} / C_{G_{\eta}}\left(U_{\eta} / V_{\eta}\right)\right) \cong E s p_{27}$ and $\left|U_{\eta} / V_{\eta}\right|=2^{6}$ for $\eta \in \mathcal{B}$.

Notation. Let $\langle\xi, \eta\rangle \in E(\Gamma)$.

$$
\begin{aligned}
& X_{\xi \eta}=C_{U_{\eta} \bmod V_{\eta}}\left(Z_{\xi \eta}\right), \quad \text { and } \\
& T_{\xi}^{*}=\left\langle X_{\xi \eta}{ }^{{ }^{*}}\right\rangle .
\end{aligned}
$$

(I-1) Let $\langle\xi, \eta\rangle \in E(\Gamma)$. Then

$$
\begin{aligned}
& G_{\xi \eta} / Q_{\eta} \cong \mathbf{Z}_{8}, \mathbf{D}_{8}, \text { or } S D_{16}, \text { and } C_{\xi} Q_{\eta} / Q_{\eta} \cong \mathbf{Z}_{4} \text { or } \mathbf{Q}_{8}, \\
& {\left[U_{\eta}, G_{\xi \eta}\right] \supset X_{\xi \eta} \supset W_{\xi},} \\
& \left|U_{\eta}:\left[U_{\eta}, G_{\xi \eta}\right]\right|=\left|\left[U_{\eta}, G_{\xi \eta}\right]: X_{\xi \eta}\right|=2, \\
& {\left[\left[U_{\eta}, G_{\xi \eta}\right], C_{\xi}\right] \varsubsetneqq W_{\xi} \supseteqq\left[X_{\xi \eta}, C_{\xi}\right], \quad \text { and }} \\
& {\left[\left[U_{\eta}, G_{\xi \eta}\right], Z_{\xi \eta}\right] \cong V_{\xi} \nsupseteq\left[X_{\xi \eta}, G_{\xi \eta}\right],}
\end{aligned}
$$

Proof. We see that this follows from (4-ii,iii) together with $(2.7)-(4)(5)$, regarding $U_{\eta} / V_{\eta}$ as a six dimensional $G F(2)\left(G_{\eta} / C_{G_{\eta}}\left(U_{\eta} / V_{\eta}\right)\right)$-module.

(I-2) Let $\langle\xi, \eta\rangle \in E(\Gamma)$. Then $W_{\xi} \subseteq T_{\xi}{ }^{*} \subseteq T_{\xi}^{(1)} \subseteq Q_{\eta}, U_{\eta} \cap T_{\xi}^{(1)}=$ $\left[U_{\eta}, G_{\xi \eta}\right]$, and $\left[T_{\xi}^{*}, C_{\xi}\right] \subseteq W_{\xi} \supseteqq\left(T_{\xi}^{*}\right)^{2}$.

Proof. We may assume $\xi=\gamma$ and $\eta=\delta$. Let $\lambda \in \Delta(\gamma)$. If $U_{\lambda} \nsubseteq Q_{\delta}$, then $(\lambda, \delta) \in \mathcal{C}^{\prime}$, so $U_{\lambda} \subseteq Z_{\gamma \delta}$ by (3). Thus $T_{\gamma}=\left\langle U_{\lambda} \mid \lambda \in \Delta(\gamma)\right\rangle \subseteq Z_{\gamma \delta}$, and so $T_{\gamma}{ }^{(1)}=\left[T_{\gamma}, Q_{\gamma}\right] \subseteq\left[Z_{\gamma \delta}, G_{\gamma \delta}\right] \subseteq Q_{\delta}$. Since $W_{\gamma}=\left[U_{\beta}, U_{\delta}\right]$ as in the proof of (4), we have $T_{\gamma}^{(1)} \supseteqq\left[U_{\beta}, U_{\delta}\right]=W_{\gamma} \supseteqq V_{\delta}$, and so $\left[U_{\delta}, G_{\gamma \delta}\right] \subseteq\left[U_{\delta}, Q_{\gamma}\right] V_{\delta}$ 
$\subseteq U_{\delta} \cap T_{\gamma}^{(1)} \subset U_{\delta}$. Thus $U_{\delta} \cap T_{\gamma}^{(1)}=\left[U_{\delta}, G_{\gamma \delta}\right] \supseteqq X_{\gamma \delta} \supseteqq W_{\gamma}$ by (I- 1$)$, and hence $T_{\gamma}{ }^{(1)}=\left\langle\left(U_{\delta} \cap T_{\gamma}{ }^{(1)}\right)^{G_{\gamma}}\right\rangle=\left\langle X_{\gamma \delta}^{G_{\gamma}}\right\rangle=T_{\gamma}{ }^{*}$ by $(2.14)-(1)$. The third assertion also holds because $\left[T_{\gamma}{ }^{*}, T_{\gamma}{ }^{*}\right] \subseteq\left[T_{\gamma}{ }^{*}, C_{\gamma}\right]=\left\langle\left[X_{\gamma \delta}, C_{\gamma}\right]^{G_{\gamma}}\right\rangle \subseteq W_{\gamma}$ and $T_{\gamma}{ }^{*} / W_{\gamma}=$ $\left\langle\left(X_{\gamma \delta} / W_{\gamma}\right)^{G_{\gamma}}\right\rangle=\Omega_{1}\left(T_{\gamma}{ }^{*} / W_{\gamma}\right)$.

$$
U_{\delta} \cap Q_{\beta}=\left[U_{\delta}, G_{\gamma \delta}\right]
$$

Proof. Since $G_{\gamma \delta}=Q_{\gamma} Q_{\delta}=\left(Q_{\beta} \cap Q_{\gamma}\right) Q_{\delta}$ by $(2),\left[U_{\delta}, G_{\gamma \delta}\right]=$ $\left[U_{\delta},\left(Q_{\beta} \cap Q_{\gamma}\right) Q_{\delta}\right]=\left[U_{\delta}, Q_{\beta} \cap Q_{\gamma}\right] W_{\gamma} \leqq U_{\delta} \cap Q_{\beta}$. Thus $U_{\delta} \cap Q_{\beta}=\left[U_{\delta}, G_{\gamma \delta}\right]$ because $\left|U_{\delta}:\left[U_{\delta}, G_{\gamma \delta}\right]\right|=2=\left|U_{\delta}: U_{\delta} \cap Q_{\beta}\right|$ by $(3)$ and (I-1).

(I-4) Let $\xi \in \mathcal{A}$. Then

$$
\left[T_{\xi}^{(1)}, O^{2}\left(G_{\xi}\right)\right] \nsubseteq T_{\xi}^{*} \quad \text { and } \quad\left[T_{\xi}^{*}, O^{2}\left(G_{\xi}\right)\right] \nsubseteq W_{\xi} .
$$

Proof. We may assume $\xi=\gamma$. Suppose first that $\left[T_{\gamma}{ }^{(1)}, O^{2}\left(G_{\gamma}\right)\right] \subseteq T_{\gamma}{ }^{*}$. Then we have $T_{\gamma}{ }^{(1)}=\left\langle\left(U_{\delta} \cap Q_{\beta}\right)^{G_{\gamma}}\right\rangle=\left(U_{\delta} \cap Q_{\beta}\right) T_{\gamma}{ }^{*}$ by (I-2), (I-3), and (2.14)(1). Since $\left[U_{\delta} \cap Q_{\beta}, C_{\gamma} \cap Q_{\delta}\right] \cong V_{\delta} \cong W_{\gamma} \supseteqq\left[T_{\gamma}{ }^{*}, C_{\gamma} \cap Q_{\delta}\right]$ by (I-2), we have $\left[T_{\gamma}{ }^{(1)}, C_{\gamma} \cap Q_{\delta}\right] \subseteq W_{\gamma}$, and hence $\left[\left[U_{\beta}, G_{\beta \gamma}\right], C_{\gamma}\right] \subseteq\left[U_{\beta} \cap T_{\gamma}{ }^{(1)},\left(Q_{\beta} \cap C_{\gamma}\right)\left(C_{\gamma} \cap Q_{\delta}\right)\right]$ $\subseteq W_{\gamma}$, contrary to (I-1).

Suppose next that $\left[T_{\gamma}{ }^{*}, O^{2}\left(G_{\gamma}\right)\right] \subseteq W_{\gamma}$. Then we have $X_{\beta \gamma}=X_{\gamma \delta} \subseteq U_{\beta} \cap U_{\delta}$, and so $\left[X_{\gamma \delta}, G_{\gamma \delta}\right] \subseteq\left[U_{\beta} \cap U_{\delta},\left\langle Q_{\beta}, Q_{\delta}\right\rangle\right] \subseteq V_{\beta} V_{\delta}=V_{\gamma}$. This also contradicts (I-1).

Now, take $\alpha \in \Delta(\beta)$ so that $V_{\alpha} \nsubseteq Q_{\delta}$.

(I-5) The following holds.

(i) $G_{\beta}=\left\langle Z_{\alpha \beta}, G_{\beta \gamma}\right\rangle$,

(ii) $U_{\delta} \cap Q_{\beta} \nsubseteq Q_{\alpha}$,

(iii) $\left[T_{\alpha}{ }^{*} \cap Z_{\gamma \delta}, U_{\delta} \cap Q_{\beta}\right] \subseteq W_{\alpha}$, and

(iv) $T_{\alpha}{ }^{*} \nsubseteq Z_{\gamma \delta}$.

Proof. Let $\widetilde{G}_{\beta}=G_{\beta} / C_{G_{\beta}}\left(U_{\beta} / V_{\beta}\right)$. Since $X_{\alpha \beta} \varsubsetneqq Q_{\delta} \supseteqq X_{\beta \gamma}$, we have $\widetilde{Z}_{\alpha \beta} \neq \widetilde{Z}_{\beta \gamma}$, so $\widetilde{G}_{\alpha \beta} \nsubseteq Z\left(O_{3}\left(\widetilde{G}_{\beta}\right)\right) \widetilde{G}_{\beta \gamma}$ because $\left[\widetilde{Z}_{\alpha \beta}, Z\left(O_{3}\left(\widetilde{G}_{\beta}\right)\right)\right]=1$ by $(2.7)-$ (1). Thus we have $\widetilde{G}_{\beta}=\left\langle\widetilde{G}_{\beta \gamma}, \widetilde{G}_{\gamma \delta}\right\rangle$ because $Z\left(O_{3}\left(\widetilde{G}_{\beta}\right)\right) \widetilde{G}_{\beta \gamma}$ is the unique maximal subgroup of $\widetilde{G}_{\beta}$ containing $\widetilde{G}_{\beta \gamma}$ by (2.1)-(8). Hence $G_{\beta}=\left\langle G_{\beta \gamma}, G_{\gamma \delta}\right\rangle$ by the 2irreducibility of $G_{\beta}$, and then (i) holds by (2.1)-(10).

Since $U_{\beta}=V_{\alpha}\left(U_{\beta} \cap Q_{\delta}\right)$ by the choice of $\alpha, V_{\alpha}$ does not induce a transvection on $U_{\delta} / V_{\delta}$ by (3), and hence (ii) holds also by (3).

Note that $X_{\alpha \beta} \subseteq U_{\beta} \cap T_{\alpha}{ }^{*} \subseteq U_{\beta} \cap T_{\alpha}{ }^{(1)}=\left[U_{\beta}, G_{\beta \gamma}\right]$. Therefore, if $U_{\beta} \cap T_{\alpha}{ }^{*} \neq$ $X_{\alpha \beta}$ then $U_{\beta} \cap T_{\alpha}{ }^{*}=U_{\beta} \cap T_{\alpha}{ }^{(1)}$ by (I-1), so $T_{\alpha}{ }^{*}=\left\langle\left(U_{\beta} \cap T_{\alpha}{ }^{(1)}\right)^{G_{\alpha}}\right\rangle=T_{\alpha}{ }^{(1)}$, a contradiction. This shows that $X_{\alpha \beta}=U_{\beta} \cap T_{\alpha}{ }^{*}$, and hence

$$
\left[T_{\alpha}{ }^{*} \cap Z_{\gamma \delta}, U_{\delta} \cap Q_{\beta}\right] \subseteq T_{\alpha}{ }^{*} \cap V_{\gamma}=T_{\alpha}{ }^{*} \cap U_{\beta} \cap V_{\gamma}=X_{\alpha \beta} \cap V_{\gamma}=V_{\beta} \leqq W_{\alpha}
$$


by (i) and (I-4). Thus, in particular, $T_{\alpha}{ }^{*} \nsubseteq Z_{\gamma \delta}$ by (ii). This proves (iii) and (iv).

(I-6) Let $\xi \in \mathcal{A}$. Then $O^{2}\left(G_{\xi} / C_{G_{\xi}}\left(T_{\xi}^{*} / W_{\xi}\right)\right) \cong \mathbf{Z}_{3}$.

Proof. Suppose first that $T_{\alpha}{ }^{*} \subseteq Q_{\gamma}$. Since $\left(T_{\alpha}{ }^{*}\right)^{2} \subseteq W_{\alpha} \subseteq Z_{\gamma \delta}$ and $G_{\gamma \delta} / Z_{\gamma \delta}$ is isomorphic to a subgroup of $\mathbf{D}_{8}$ by (I-1) and (I-2), we have

$$
\left|T_{\alpha}{ }^{*}: T_{\alpha}{ }^{*} \cap Z_{\gamma \delta}\right| \leqq 2^{2}
$$

and so $U_{\delta} \cap Q_{\beta}$ induces a quasitransvection on $T_{\alpha}{ }^{*} / W_{\alpha}$.

Suppose next that $T_{\alpha}{ }^{*} \nsubseteq Q_{\gamma}$. Then

$$
\left[T_{\alpha}^{*}, T_{\gamma}^{(1)} \cap C_{\alpha}\right] \subseteq W_{\alpha} \cap T_{\gamma}^{(1)} \subseteq W_{\alpha} \cap Q_{\delta} \neq W_{\alpha}
$$

by (I-2) and the choice of $\alpha$, so $\left|\left[T_{\alpha}{ }^{*}, T_{\gamma}^{(1)} \cap C_{\alpha}\right] W_{\gamma}: W_{\gamma}\right| \leqq 2$. Also, $\left|T_{\gamma}{ }^{(1)}: T_{\gamma}{ }^{(1)} \cap C_{\alpha}\right| \leqq\left|Q_{\beta}: C_{\alpha} \cap Q_{\beta}\right|=2^{2}$ by (4-iii) and (I-2), and hence

$$
\left|T_{\gamma}{ }^{(1)}: T_{\gamma}^{(1)} \cap C_{\alpha}\right| \cdot\left|\left[T_{\alpha}{ }^{*}, T_{\gamma}^{(1)} \cap C_{\alpha}\right] W_{\gamma}: W_{\gamma}\right| \leqq 2^{3} .
$$

Therefore $T_{\alpha}{ }^{*}$ induces a factorwise quasitransvection on $T_{\gamma}^{(1)} / W_{\gamma}$ by (I-4).

Thus, letting $\xi=\alpha$ or $\gamma$, we have $O^{2}\left(G_{\xi} / Q_{\xi} C_{G_{\xi}}\left(T_{\xi}{ }^{*} / W_{\xi}\right)\right) \cong \mathbf{Z}_{3}$ in either case by $(2.11)-(1)$ because $G_{\xi}$ is a $\{2,3\}$-group with cyclic Sylow 3 -subgroups.

(I-7) A contradiction.

Proof. By (I-6), $T_{\gamma}{ }^{*}=\left\langle X_{\beta \gamma}, X_{\gamma \delta}, X_{\gamma \delta}{ }^{t}\right\rangle$ for some $t \in G_{\beta \gamma}-Q_{\gamma}$. Note that $\left[U_{\beta}, X_{\beta \gamma}\right]=1$, that $\left[U_{\beta}, X_{\gamma \delta}\right] \subseteq V_{\beta} \cap V_{\delta}=1$, and that $\left[U_{\beta}, X_{\beta \gamma}{ }^{t}\right]=\left[U_{\beta}, X_{\beta \gamma}\right]^{t}=1$. Thus we have $\left[U_{\beta}, T_{\gamma}{ }^{*}\right]=1$, and hence $\left[T_{\gamma}, T_{\gamma}{ }^{*}\right]=\left[\left\langle U_{\beta}{ }^{G_{\gamma}}\right\rangle, T_{\gamma}{ }^{*}\right]=1$, and, in particular, $T_{\gamma}{ }^{*}$ is elementary abelian. Since $T_{\alpha}{ }^{*} \subseteq C_{Q_{\beta}}\left(U_{\beta}\right) \subseteq C_{Q_{\gamma}}\left(W_{\gamma}\right) \subseteq C_{\gamma}$, we have $T_{\alpha}{ }^{*} \subseteq \Omega_{1}\left(C_{\gamma}\right) \subseteq Z_{\gamma \delta}$ by (I-1), which contradicts (iv) of (I-5).

Case II. $O^{2}\left(G_{\eta} / C_{G_{\eta}}\left(U_{\eta} / V_{\eta}\right)\right) \cong \mathbf{E}_{3^{2}}$ and $\left|U_{\eta} / V_{\eta}\right|=2^{4}$ for $\eta \in \mathcal{B}$.

$$
U_{\beta} \cap Q_{\delta}=\left[U_{\beta}, G_{\beta \gamma}\right]=\left[U_{\beta}, Q_{\gamma}\right]=\left[U_{\beta}, Q_{\gamma} \cap Q_{\delta}\right] .
$$

Proof. We have $\left|U_{\beta}:\left[U_{\beta}, G_{\beta \gamma}\right]\right|=2$ by $(2.5)-(3)$, regarding $U_{\beta} / V_{\beta}$ as a four dimensional $G F(2)\left(G_{\beta} / C_{G_{\beta}}\left(U_{\beta} / V_{\beta}\right)\right)$-module, so the assertion follows as in the proof of (I-3).

(II-2) Let $\eta \in \mathcal{B}$. Then $O^{2}\left(G_{\eta} / C_{G_{\eta}}\left(Q_{\eta} / C_{Q_{\eta}}\left(U_{\eta}\right)\right)\right) \cong \mathbf{E}_{3^{2}}, Q_{\eta} / C_{Q_{\eta}}\left(U_{\eta}\right) \cong$ $\mathbf{E}_{2^{4}}$, and $\left[C_{Q_{\eta}}\left(U_{\eta}\right), O^{2}\left(G_{\eta}\right)\right] \subseteq U_{\eta}$.

Proof. We may assume $\eta=\delta$. Take $\lambda \in \Delta(\delta)$ so that $U_{\delta}=W_{\gamma} W_{\lambda}$. (This is possible by (2.5)-(11).) Then $C_{Q_{\delta}}\left(U_{\delta}\right)=\left(C_{\gamma} \cap Q_{\delta}\right) \cap\left(Q_{\delta} \cap C_{\lambda}\right) \cong$ $Q_{\gamma}$, so $\left|Q_{\delta} / C_{Q_{\delta}}\left(U_{\delta}\right)\right| \leqq 2^{4}$ by (4-iii) and $\left[Q_{\delta}, O^{2}\left(G_{\delta}\right)\right] \varsubsetneqq C_{Q_{\delta}}\left(U_{\delta}\right)$ by (3.2)-(2). Since $\left[U_{\beta}, C_{\gamma} \cap Q_{\delta}\right] V_{\beta} \subseteq W_{\gamma} \subseteq U_{\delta} \subseteq C_{Q_{\delta}}\left(U_{\delta}\right)$ by $(2.5)-(4),\left[U_{\beta}, C_{Q_{\delta}}\left(U_{\delta}\right)\right] \subseteq U_{\delta}$ and 
$\left|Q_{\delta} / C_{Q_{\delta}}\left(U_{\delta}\right): C_{Q_{\delta} / C_{Q_{\delta}}\left(U_{\delta}\right)}\left(U_{\beta}\right)\right| \leqq 2^{2}$ because $U_{\beta}$ does not induce a factorwise transvection on $Q_{\delta}$ by (3). Therefore $\left[C_{Q_{\delta}}\left(U_{\delta}\right), O^{2}\left(G_{\delta}\right)\right] \cong U_{\delta}$, and (2.9) shows that $Q_{\delta} / C_{Q_{\delta}}\left(U_{\delta}\right) \cong \mathbf{E}_{2^{4}}$ and that $O^{2}\left(G_{\delta} / C_{G_{\delta}}\left(Q_{\delta} / C_{Q_{\delta}}\left(U_{\delta}\right)\right)\right) \cong \mathbf{E}_{3^{2}}$.

Now, define $R_{\eta}=\left[Q_{\eta}, O^{2}\left(G_{\eta}\right)\right] U_{\eta}$ and $L_{\eta}=C_{R_{\eta}}\left(O^{2}\left(G_{\eta}\right)\right)$ for $\eta \in \mathcal{B}$.

(II-3) $\quad R_{\delta} \supseteqq U_{\beta} \cap Q_{\delta} \nsubseteq C_{R_{\delta}}\left(U_{\delta}\right)$.

Proof. Since $\left[U_{\beta}, C_{Q_{\delta}}\left(U_{\delta}\right)\right] \subseteq\left[U_{\beta}, C_{\gamma}\right] \subseteq W_{\gamma} \subseteq U_{\delta} \subseteq R_{\delta}$ by (2.5)-(4), we have $\left[U_{\beta}, Q_{\delta}\right]=\left[U_{\beta}, C_{Q_{\delta}}\left(U_{\delta}\right) R_{\delta}\right] \subseteq R_{\delta}$. Therefore $U_{\beta} \cap Q_{\delta}=\left[U_{\beta}, Q_{\gamma} \cap Q_{\delta}\right] \subseteq$ $R_{\delta}$ by (II- 1$)$ and $\left[U_{\beta} \cap Q_{\delta}, U_{\delta}\right] \nsubseteq V_{\beta}$ by (3).

Let $P \in \operatorname{Syl}_{3}\left(G_{\delta}\right)$. Then

$$
\begin{aligned}
& \Phi(P) Q_{\delta}=C_{G_{\delta}}\left(U_{\delta} / V_{\delta}\right)=C_{G_{\delta}}\left(Q_{\delta} / C_{Q_{\delta}}\left(U_{\delta}\right)\right)=Q_{\delta} C_{G_{\delta}}\left(Q_{\delta}\right) \text { and } \\
& P / \Phi(P) \cong \mathbf{E}_{3^{2}} .
\end{aligned}
$$

Take two subgroups $P_{1}$ and $P_{2}$ of $P$ so that

$$
\begin{aligned}
& P / \Phi(P)=P_{1} \Phi(P) / \Phi(P) \times P_{2} \Phi(P) / \Phi(P) \quad \text { and } \\
& \left|C_{Q_{\delta} / C_{Q_{\delta}}\left(U_{\delta}\right)}\left(P_{1}\right)\right|=\left|C_{Q_{\delta} / C_{Q_{\delta}}\left(U_{\delta}\right)}\left(P_{2}\right)\right|=2^{2} .
\end{aligned}
$$

$$
\left|C_{U_{\delta} / V_{\delta}}\left(P_{i}\right)\right|=2^{2}(i=1,2) .
$$

Proof. Suppose false. Since $P_{1} \Phi(P) Q_{\delta}$ and $P_{2} \Phi(P) Q_{\delta}$ are conjugate in $G_{\delta}$ by the 2-irreducibility of $G_{\delta}$, we have $U_{\delta}=\left[U_{\delta}, P\right] V_{\delta}=\left[U_{\delta}, P_{i}\right] V_{\delta}(i=1,2)$. Also, since $\left[P_{i}, C_{Q_{\delta}}\left(P_{i}\right), U_{\delta}\right]=1=\left[V_{\delta}, P_{i}\right] \supseteq\left[C_{Q_{\delta}}\left(P_{i}\right), U_{\delta}, P_{i}\right](i=1,2)$, the threesubgroup-lemma shows that $\left[U_{\delta}, P_{i}, C_{Q_{\delta}}\left(P_{i}\right)\right]=1(i=1,2)$. Therefore, since $Q_{\delta}=$ $\left\langle C_{Q_{\delta}}\left(U_{\delta}\right), C_{Q_{\delta}}\left(P_{1}\right), C_{Q_{\delta}}\left(P_{2}\right)\right\rangle$ by the choice of $P_{1}$ and $P_{2}$, we have $\left[U_{\delta}, Q_{\delta}\right]=1$, a contradiction.

(II-5) Let $\eta \in \mathcal{B}$. Then $R_{\eta} / U_{\eta} \cong \mathbf{E}_{2^{4}}$ or $\mathbf{Q}_{8} * \mathbf{Q}_{8}$. (We denote by $*$ the central product.)

Proof. We may assume $\eta=\delta$. Since $G_{\delta}=Q_{\delta} P N_{G_{\delta}}(P)$ by a Frattini argument, we have $\left[Q_{\delta}, P\right] U_{\delta} \triangleleft G_{\delta}$, so $R_{\delta}=\left[Q_{\delta}, P\right] U_{\delta}$.

Now, let $\bar{Q}_{\delta}=Q_{\delta} / U_{\delta}$. Note that $C_{\bar{Q}_{\delta}}(P)=\overline{C_{Q_{\delta}}\left(U_{\delta}\right)} \triangleleft \bar{Q}_{\delta}$ by (II-2), and hence $\left[P, \overline{C_{Q_{\delta}}\left(U_{\delta}\right)}, \bar{Q}_{\delta}\right]=1=\left[\overline{C_{Q_{\delta}}\left(U_{\delta}\right)}, \bar{Q}_{\delta}, P\right]$. Therefore the three-subgrouplemma shows that $\left[\bar{R}_{\delta}, \overline{C_{Q_{\delta}}\left(U_{\delta}\right)}\right]=\left[\bar{Q}_{\delta}, P, \overline{C_{Q_{\delta}}\left(U_{\delta}\right)}\right]=1$ so $\bar{Q}_{\delta}=\bar{R}_{\delta} * \overline{C_{Q_{\delta}}\left(U_{\delta}\right)}$. Moreover, as $C_{\overline{R_{\delta}}}\left(P_{i}\right) \triangleleft \bar{R}_{\delta}(i=1,2)$, the same argument also shows that $\bar{R}_{\delta}=$ $\left[\bar{R}_{\delta}, P_{i}\right] * C_{\bar{R}_{\delta}}\left(P_{i}\right)(i=1,2)$. Since $C_{\bar{R}_{\delta}}\left(P_{i}\right)=C_{\bar{R}_{\delta}}(P) *\left[\bar{R}_{\delta}, P_{3-i}\right](i=1,2)$, we conclude that $\bar{R}_{\delta}=C_{\bar{R}_{\delta}}(P)\left[\bar{R}_{\delta}, P_{1}\right]\left[\bar{R}_{\delta}, P_{2}\right]=C_{\bar{R}_{\delta}}\left(P_{1}\right) * C_{\bar{R}_{\delta}}\left(P_{2}\right)$. Since $\overline{C_{R_{\delta}}\left(U_{\delta}\right)}$ $\subseteq Z\left(\bar{R}_{\delta}\right)$ and $\left|C_{\bar{R}_{\delta}}\left(P_{i}\right): \overline{C_{R_{\delta}}\left(U_{\delta}\right)}\right|=2^{2}(i=1,2)$, we have $\left|\left[C_{\bar{R}_{\delta}}\left(P_{i}\right), C_{\bar{R}_{\delta}}\left(P_{i}\right)\right]\right| \leqq 2$ 
$(i=1,2)$, and hence $C_{\bar{R}_{\delta}}\left(P_{i}\right) \cong \mathbf{E}_{2^{2}}$ or $\mathbf{Q}_{8}$. Consequently, we have $\bar{R}_{\delta} \cong \mathbf{E}_{2^{4}}$ or $\mathrm{Q}_{8} * \mathrm{Q}_{8}$ because $\Omega_{1}\left(\bar{R}_{\delta}\right) \supseteqq \overline{U_{\beta} \cap Q_{\delta}} \varsubsetneqq \overline{C_{R_{\delta}}\left(U_{\delta}\right)}=\left[\bar{R}_{\delta}, \bar{R}_{\delta}\right]=\Phi\left(\bar{R}_{\delta}\right)$ by (II- 1$)$ and the definition of $R_{\delta}$.

(II-6) Let $\eta \in \mathcal{B}$. Then $R_{\eta} / L_{\eta} \cong \mathbf{E}_{2^{8}}$.

Proof. We may assume $\eta=\delta$. Note that $G_{\delta}=Q_{\delta} N_{G_{\delta}}(P)=R_{\delta} N_{G_{\delta}}(P)$. Since $U_{\delta} / V_{\delta} \subseteq Z\left(R_{\delta} / V_{\delta}\right)$ and $R_{\delta}=\langle X| C_{R_{\delta}}\left(U_{\delta}\right) \subseteq X$ and $X / U_{\delta}$ is cyclic $\rangle$, $R_{\delta}$ is generated by a set of abelian subgroups containing $C_{R_{\delta}}\left(U_{\delta}\right)$, and hence $C_{R_{\delta}}\left(U_{\delta}\right) / V_{\delta} \subseteq Z\left(R_{\delta} / V_{\delta}\right)$. Since $V_{\delta} \leqq C_{R_{\delta}}(P) \subseteq C_{R_{\delta}}\left(U_{\delta}\right)$, we have $C_{R_{\delta}}(P) \triangleleft G_{\delta}$, and so $L_{\delta}=C_{R_{\delta}}(P)$. Hence $\left|R_{\delta} / L_{\delta}\right|=2^{8}$.

Now, let $\widehat{R}_{\delta}=R_{\delta} / L_{\delta}$ and $\widetilde{P}=P / \Phi(P)$. Then $\widehat{R}_{\delta}=C_{\widehat{R}_{\delta}}\left(\widetilde{P}_{1}\right) C_{\widehat{R}_{\delta}}\left(\widetilde{P}_{2}\right)$ and $\widetilde{P}=\widetilde{P}_{1} \times \widetilde{P}_{2}$. Since $C_{\widehat{R}_{\delta}}\left(\widetilde{P}_{1}\right) \cap C_{\widehat{R}_{\delta}}\left(\widetilde{P}_{2}\right)=C_{\widehat{R}_{\delta}}(\widetilde{P})=1$, we have $\widehat{R}_{\delta}=C_{\widehat{R}_{\delta}}\left(\widetilde{P}_{1}\right) \times$ $C_{\widehat{R}_{\delta}}\left(\widetilde{P}_{2}\right)$ by $(1.4)$, so $C_{\widehat{R}_{\delta}}\left(\widetilde{P}_{i}\right) \cong \mathbf{E}_{2^{4}}$ or $\mathbf{Z}_{4} \times \mathbf{Z}_{4}(i=1,2)$. Thus $\widehat{R}_{\delta} \cong \mathbf{E}_{2^{8}}$ or $\mathbf{Z}_{4} \times \mathbf{Z}_{4} \times \mathbf{Z}_{4} \times \mathbf{Z}_{4}$. In the latter case, we have $\Omega_{1}\left(R_{\delta}\right) \subseteq C_{R_{\delta}}\left(U_{\delta}\right)$, contrary to (II-3). Therefore $R_{\delta} / L_{\delta} \cong \mathbf{E}_{2^{8}}$.

$$
O^{2}\left(G_{\gamma} / C_{G_{\gamma}}\left(T_{\gamma}^{(1)} / W_{\gamma}\right)\right) \cong \mathbf{Z}_{3}, T_{\gamma}^{(1)} \subseteq R_{\delta} \text {, and }\left|T_{\gamma}^{(1)} / W_{\gamma}\right|=2^{3} \text {. }
$$

Proof. Since $U_{\beta} \cap T_{\gamma}{ }^{(1)}=U_{\beta} \cap Q_{\delta}$ by (II- 1$),\left[T_{\gamma}{ }^{(1)}, U_{\delta}\right] \neq 1$, and hence we can choose $\lambda \in \Delta(\delta)$ and $\mu \in \Delta(\lambda)$ so that $T_{\gamma}^{(1)} \nsubseteq Q_{\lambda}$ and $(\delta, \mu) \in \mathcal{C}^{\prime}$. Then $\left[T_{\gamma}^{(1)}, T_{\lambda}{ }^{(1)} \cap Q_{\gamma}\right] \subseteq W_{\gamma} \cap T_{\lambda}{ }^{(1)} \subseteq U_{\delta} \cap T_{\lambda}^{(1)}$ and $\left|T_{\lambda}{ }^{(1)}: T_{\lambda}{ }^{(1)} \cap Q_{\gamma}\right| \leqq \mid G_{\gamma \delta}:$ $Q_{\gamma}|=2=| U_{\delta} \cap T_{\lambda}^{(1)}: W_{\lambda} \mid$, so $T_{\lambda}^{(1)}$ induces a quasitransvection on $T_{\lambda}^{(1)} / W_{\lambda}$. Thus $O^{2}\left(G_{\lambda} / C_{G_{\lambda}}\left(T_{\lambda}^{(1)} / W_{\lambda}\right)\right) \cong \mathbf{Z}_{3}$, and hence $T_{\gamma}^{(1)}=\left\langle\left(U_{\beta} \cap T_{\gamma}^{(1)}\right)^{G_{\gamma}}\right\rangle=$ $\left\langle\left(U_{\beta} \cap Q_{\delta}\right)^{G_{\gamma \delta}}, U_{\delta} \cap T_{\gamma}^{(1)}\right\rangle \subseteq R_{\delta}$ by (II-3).

If $L_{\delta}=V_{\delta}$, then $R_{\delta}{ }^{\prime} \subseteq V_{\delta} \subseteq W_{\gamma}$, so $\left[T_{\gamma}{ }^{(1)}, R_{\delta}\right] \subseteq W_{\gamma}$, a contradiction. Therefore $L_{\delta} \neq V_{\delta}$, and so $R_{\delta} / U_{\delta} \cong \mathrm{Q}_{8} * \mathrm{Q}_{8}$. Now, suppose $\left|T_{\gamma}^{(1)} / W_{\gamma}\right|=2^{2}$. Then $\left|T_{\gamma}{ }^{(1)} U_{\delta} / U_{\delta}\right|=2$, so $T_{\gamma}{ }^{(1)} U_{\delta} / U_{\delta} \subseteq Z\left(R_{\delta} / U_{\delta}\right)=C_{R_{\delta}}\left(U_{\delta}\right) / U_{\delta}$. Therefore $U_{\beta} \cap Q_{\delta} \subseteq T_{\gamma}^{(1)} \subseteq C_{R_{\delta}}\left(U_{\delta}\right)$, also a contradiction. Thus $\left|T_{\gamma}{ }^{(1)} / W_{\gamma}\right|=2^{3}$.

(II-8) A contradiction.

Proof. Since $\left[T_{\gamma}^{(1)}, Q_{\gamma}\right] \subseteq W_{\gamma} \subseteq C_{R_{\delta}}\left(U_{\delta}\right)$, we have $\left|T_{\gamma}^{(1)}: T_{\gamma}^{(1)} \cap C_{R_{\delta}}\left(U_{\delta}\right)\right|$ $=\left|T_{\gamma}{ }^{(1)} C_{R_{\delta}}\left(U_{\delta}\right): C_{R_{\delta}}\left(U_{\delta}\right)\right|=2$, and so $\left|C_{R_{\delta}}\left(U_{\delta}\right): T_{\gamma}{ }^{(1)} \cap C_{R_{\delta}}\left(U_{\delta}\right)\right|=2$. Let $N_{\gamma}=C_{R_{\beta}}\left(U_{\beta}\right) \cap T_{\gamma}{ }^{(1)} \cap C_{R_{\delta}}\left(U_{\delta}\right)$. Then $\left|T_{\gamma}^{(1)} / N_{\gamma}\right|=2^{2}=\left|C_{R_{\delta}}\left(U_{\delta}\right) N_{\gamma}\right|$. Choose $\lambda \in \Delta(\delta)$ and $\mu \in \Delta(\lambda)$ so that $(\delta, \mu) \in \mathcal{C}^{\prime}$ and $\left\langle U_{\beta}, U_{\mu}\right\rangle$ has a cyclic 3-subgroup $X$ with $C_{C_{R_{\delta}}\left(U_{\delta}\right)}(X)=L_{\delta}$. (This is possible by (II-4) and the choice of $P_{1}$ and $\left.P_{2}\right)$. Then $N_{\gamma} \cap N_{\lambda} \subseteq C_{C_{R_{\delta}}\left(U_{\delta}\right)}(X)=L_{\delta}$ and $\left|C_{R_{\delta}}\left(U_{\delta}\right): N_{\gamma} \cap N_{\lambda}\right| \leqq \mid C_{R_{\delta}}\left(U_{\delta}\right)$ : $L_{\delta} \mid=2^{4}$. Therefore $L_{\delta}=N_{\gamma} \cap N_{\lambda} \subseteq N_{\gamma}$, so, by symmetry, $L_{\beta} \subseteq N_{\gamma}$. Thus, since $\left[N_{\gamma}, Q_{\beta}\right] \subseteq L_{\beta} \subseteq N_{\gamma} \supseteqq L_{\delta} \supseteq\left[N_{\gamma}, Q_{\delta}\right], N_{\gamma} \triangleleft\left\langle Q_{\beta}, Q_{\delta}\right\rangle=G_{\gamma}$, and so $\left[T_{\gamma}^{(1)}, O^{2}\left(G_{\gamma}\right)\right] \varsubsetneqq N_{\gamma}$. However, by (II-6) and (II-7), we have $\left[T_{\gamma}{ }^{(1)}, R_{\delta}\right] \subseteq L_{\delta} \subseteq$ 
$N_{\gamma}$, a contradiction.

\section{$\S 8$. Odd case with $b \geqq 5$}

In this final section, we will argue for a contradiction under the following hypothesis.

HyPOTHESIS 8.1

(1) The parameter $b$ is odd and $b \geqq 5$.

(2) $n_{*}\left(G_{\eta}\right)>1$ for $\eta \in \mathcal{B}$.

Notation. Let $\beta, \delta \in V(\Gamma)$. We denote by $(\beta, \beta+1, \ldots, \delta-1, \delta)$ a path of length $d(\beta, \delta)$ from $\beta$ through $\delta$.

We will first prove the following.

Proposition 8.2 Part (2) of (6.5) holds.

Proof. We will assume otherwise, and derive a contradiction.

(1) Let $(\beta, \delta) \in \mathcal{C}^{\prime}$ and $\alpha \in \Delta(\beta)$. If $V_{\alpha} \nsubseteq Q_{\delta}$, then $G_{\beta}=\left\langle G_{\alpha \beta}, U_{\delta}\right\rangle$.

Proof. Take $(\beta, \delta) \in \mathcal{C}^{\prime}$ and $\alpha \in \Delta(\beta)$ so that $V_{\alpha} \nsubseteq Q_{\delta}$. Let $D_{\beta}=$ $C_{G_{\beta}}\left(U_{\beta} / V_{\beta}\right), \widetilde{G}_{\beta}=G_{\beta} / D_{\beta}$, and $\widehat{U}_{\beta}=\left[U_{\beta} / V_{\beta}, O^{2}\left(\widetilde{G}_{\beta}\right)\right]$. Note that $n_{*}\left(\widetilde{G}_{\beta}\right)>1$ by (2.1)-(9) and (8.1)-(2). If $n\left(G_{\beta}, \widehat{U}_{\beta}\right) \geqq 2$, then $\widetilde{U}_{\delta}$ induces a factorwise transvection on $\widehat{U}_{\beta}$ by $(6.5)-(1)$, and so $O^{2}\left(\widetilde{G}_{\beta}\right) \cong \mathbf{E}_{3^{2}}$ by $(2.11)-(1)$. Suppose $n\left(G_{\beta}, \widehat{U}_{\beta}\right)=1$. Then $\widetilde{U}_{\delta}$ induces a quasitransvection on the irreducible faithful $G F(2) \widetilde{G}_{\beta}$-module $\widehat{U}_{\beta}$ by $(2.4)-(2)(3)$ and $(6.5)-(1)$. Thus we have $O^{2}\left(\widetilde{G}_{\beta}\right) \cong \mathbf{E}_{3^{2}}, \mathbf{E}_{5^{2}}$, or $E s p_{27}$ by (2.9), and, in particular, $\widetilde{U}_{\delta}=\Omega_{1}\left(Z\left(\widetilde{G}_{\beta, \beta+1}\right)\right)$ if $O^{2}\left(\widetilde{G}_{\beta}\right) \cong E s p_{27}$.

Suppose $G_{\beta} \neq\left\langle G_{\alpha \beta}, U_{\delta}\right\rangle$. Then $\widetilde{G}_{\beta} \neq\left(\widetilde{G}_{\alpha \beta}, \widetilde{U}_{\delta}\right\rangle$ by the 2-irreducibility of $G_{\beta}$. Note that the unique maximal subgroup of $\widetilde{G}_{\beta}$ containing $\widetilde{G}_{\alpha \beta}$ is

$$
\begin{aligned}
& \widetilde{G}_{\alpha \beta} \text { if } O^{2}\left(\widetilde{G}_{\beta}\right) \cong \mathbf{E}_{3^{2}} \text { or } \mathbf{E}_{5^{2}}, \text { and } \\
& \widetilde{G}_{\alpha \beta} Z\left(O^{2}\left(\widetilde{G}_{\beta}\right)\right) \text { if } O^{2}\left(\widetilde{G}_{\beta}\right) \cong E s p_{27}
\end{aligned}
$$

by (2.1)-(8). Thus we have $\widetilde{U}_{\delta} \subseteq \widetilde{G}_{\alpha \beta}$ if $O^{2}\left(\widetilde{G}_{\beta}\right) \cong \mathbf{E}_{3^{2}}$ or $\mathbf{E}_{5^{2}}$. Suppose $O^{2}\left(\widetilde{G}_{\beta}\right) \cong$ $E s p_{27}$. Then, as $\left[\widetilde{U}_{\delta}, Z\left(O^{2}\left(\widetilde{G}_{\beta}\right)\right)\right]=1$ by $(2.7)-(1)$, we have $\widetilde{U}_{\delta} \subseteq \widetilde{G}_{\alpha \beta}$ also in this case. This shows that $U_{\delta} \subseteq G_{\alpha \beta} D_{\beta}$. Therefore $U_{\delta} \subseteq G_{\alpha \beta}{ }^{x}$ and $V_{\alpha}=V_{\alpha}{ }^{x}$ for some $x \in G_{\beta}$ as in the proof of (1) of (3.10). Thus we have $U_{\beta}=V_{\alpha}\left(U_{\beta} \cap Q_{\delta}\right)=$ $V_{\alpha}{ }^{x}\left(U_{\beta} \cap Q_{\delta}\right)$ and $\left|U_{\delta}: U_{\delta} \cap Q_{\alpha}{ }^{x}\right| \leqq\left|G_{\alpha \beta}{ }^{x}: Q_{\alpha}{ }^{x}\right| \leqq 2$, and then $U_{\beta}$ induces a transvection on $U_{\delta} / V_{\delta}$, contrary to (6.5)-(1).

Define $T_{\xi}=\left\langle U_{\eta} \mid \eta \in \Delta(\xi)\right\rangle$ for $\xi \in \mathcal{A}$.

Note that $T_{\xi}$ is elementary abelian by the minimality of $b$. 
(2) There exist $(\beta, \delta) \in \mathcal{C}^{\prime}$ and $\alpha \in \Delta(\beta)$ such that $V_{\alpha} \nsubseteq Q_{\delta}$ and $T_{\alpha} \leqq$ $Q_{\delta-2}$.

Proof. Suppose false. Let $(\beta, \delta) \in \mathcal{C}^{\prime}$ and $\alpha \in \Delta(\beta)$ so that $V_{\alpha} \nsubseteq Q_{\delta}$. Then $T_{\alpha} \nsubseteq Q_{\delta-2}$ by our hypothesis. Take $\theta \in \Delta(\alpha)$ so that $U_{\theta} \nsubseteq Q_{\delta-2}$. Then $(\theta, \delta-2) \in \mathcal{C}^{\prime}$, and so $V_{\theta} \cong U_{\delta-2} \cong T_{\delta-1}$ by $(6.5)-(1)$. Since $G_{\beta}=\left\langle G_{\alpha \beta}, U_{\delta}\right\rangle=$ $\left\langle G_{\alpha \beta}, T_{\delta-1}\right\rangle=\left\langle Q_{\alpha}, T_{\delta-1}\right\rangle$ by (1) and (3.2)-(3), we have $V_{\theta} \subseteq Z\left(G_{\beta}\right)$, and so $V_{\theta}=V_{\beta}$. Therefore, after repeated application of this "shift" operation, we can take $(\beta, \delta) \in \mathcal{C}^{\prime}$ such that $V_{\beta}=V_{\beta+2}=\cdots=V_{\delta-2}=V_{\delta}$, contrary to (6.5)-(1).

Now, take $(\beta, \delta) \in \mathcal{C}^{\prime}$ and $\alpha \in \Delta(\beta)$ so that

$$
V_{\alpha} \varsubsetneqq Q_{\delta}, G_{\beta}=\left\langle G_{\alpha \beta}, U_{\delta}\right\rangle, \quad \text { and } \quad T_{\alpha} \sqsubseteq Q_{\delta-2} \text {. }
$$

$$
U_{\delta} \cap Q_{\beta} \nsubseteq Q_{\alpha} \text { and } T_{\alpha} \nsubseteq Q_{\delta-1} \text {. }
$$

Proof. Since $V_{\alpha}$ does not induce a transvection on $U_{\delta} / V_{\delta}$ and $\mid U_{\delta}: U_{\delta} \cap$ $Q_{\beta} \mid=2$ by $(6.5)-(1)$, we have $U_{\delta} \cap Q_{\beta} \nsubseteq Q_{\alpha}$. Suppose $T_{\alpha} \subseteq Q_{\delta-1}$. If (2.13)-(1) holds, then (6.5)-(2) holds by (2.10)-(1), contrary to our hypothesis. Hence we have

$$
\left[T_{\alpha}, U_{\delta}\right] \subseteq\left[U_{\beta}, U_{\delta}\right]\left[T_{\alpha}, C_{U_{\delta} \bmod V_{\delta}}\left(U_{\beta}\right)\right] V_{\delta} \subseteq T_{\alpha}
$$

by (2.13)-(2) and (6.5)-(1). Therefore $T_{\alpha} \triangleleft\left\langle G_{\alpha \beta}, U_{\delta}\right\rangle=G_{\beta}$, a contradiction.

(4) $O^{2}\left(G_{\alpha} / C_{G_{\alpha}}\left(T_{\alpha} / V_{\alpha}\right)\right) \cong \mathbf{Z}_{3}$.

Proof. It suffices to show that $U_{\delta} \cap Q_{\beta}$ induces a factorwise quasitransvection on $T_{\alpha} / V_{\alpha}$ by (2.11)-(1) because $G_{\alpha}$ is a $\{2,3\}$-group with cyclic Sylow 3 subgroups.

Since $\left[U_{\beta}, U_{\delta} \cap Q_{\beta}\right] \subseteq V_{\beta} \subseteq V_{\alpha}$ and $\left[T_{\alpha} \cap Q_{\delta}, U_{\delta}\right] \subseteq V_{\delta}$, we have $\left[U_{\beta}\left(T_{\alpha} \cap\right.\right.$ $\left.\left.Q_{\delta}\right), U_{\delta} \cap Q_{\beta}\right] \subseteq V_{\alpha} V_{\delta}$. Thus

$$
\begin{aligned}
& \left|T_{\alpha} / V_{\alpha}:\left[U_{\beta}\left(T_{\alpha} \cap Q_{\delta-1} \cap Q_{\delta}\right) / V_{\alpha}, U_{\delta} \cap Q_{\beta}\right]\right| \\
& \quad \leqq\left|T_{\alpha}: T_{\alpha} \cap Q_{\delta-1}\right| \cdot\left|T_{\alpha} \cap Q_{\delta-1}: U_{\beta}\left(T_{\alpha} \cap Q_{\delta-1} \cap Q_{\delta}\right)\right| \cdot\left|V_{\alpha} V_{\delta}: V_{\alpha}\right| \\
& \quad \leqq 2 \cdot 2 \cdot 2 .
\end{aligned}
$$

If $n\left(G_{\alpha}, T_{\alpha} / V_{\alpha}\right)>1$, then $U_{\delta} \cap Q_{\beta}$ induces a factorwise sesquitransvection on $T_{\alpha} / V_{\alpha}$ by $(2.11)-(2)$.

Suppose $n\left(G_{\alpha}, T_{\alpha} / V_{\alpha}\right)=1$. Then we have $\left[T_{\alpha}, Q_{\alpha}\right] \subseteq U_{\beta}$ by (2.14)-(2), and so $\left[T_{\alpha}, Q_{\alpha}, Q_{\beta}\right] \subseteq V_{\beta} \subseteq V_{\alpha}$. Thus $\left[T_{\alpha}, Q_{\alpha}, G_{\alpha}\right] \subseteq\left[T_{\alpha}, Q_{\alpha},\left\langle Q_{\beta}^{G_{\alpha}}\right\rangle\right] \subseteq$ $\left\langle\left[T_{\alpha}, Q_{\alpha}, Q_{\beta}\right]^{G_{\alpha}}\right\rangle \subseteq V_{\alpha}$, and hence $\left[U_{\beta}, G_{\alpha \beta} ; 2\right]=\left[U_{\beta}, Q_{\alpha}, G_{\alpha \beta}\right] \subseteq V_{\alpha}$. Thus $\left[U_{\beta}, G_{\alpha \beta} ; 3\right] \leqq V_{\beta}$ and $\left|\left[U_{\beta}, G_{\alpha \beta} ; 2\right] V_{\beta}: V_{\beta}\right| \leqq 2^{2}$ by $(6.2)$, so $O^{2}\left(G_{\beta} / C_{G_{\beta}}\left(U_{\beta} / V_{\beta}\right)\right)$ 
$\cong E s p_{27}$ and $\left|\left[U_{\beta} / V_{\beta}, O^{2}\left(G_{\beta}\right)\right]\right|=2^{6}$ by $(2.12)$ and $(8.1)-(2)$. Since $\left[U_{\delta}, U_{\beta}, T_{\alpha} \cap\right.$ $\left.Q_{\delta-1}\right]=1$, we have $\left[U_{\delta} / V_{\delta}, Z_{\delta-1, \delta},\left(T_{\alpha} \cap Q_{\delta-1}\right) Q_{\delta}\right]=1$ by $(6.5)-(1)$, and so $T_{\alpha} \cap Q_{\delta-1}=Z_{\delta-1, \delta}=U_{\beta}\left(T_{\alpha} \cap Q_{\delta-1} \cap Q_{\delta}\right)$ by (2.7)-(6). This shows that

$$
\left|T_{\alpha} / V_{\alpha}:\left[U_{\beta}\left(T_{\alpha} \cap Q_{\delta-1} \cap Q_{\delta}\right) / V_{\alpha}, U_{\delta} \cap Q_{\beta}\right]\right| \leqq 2^{2}
$$

and so $U_{\delta} \cap Q_{\beta}$ induces a factorwise quasitransvection on $T_{\alpha} / V_{\alpha}$.

(5) A contradiction.

Proof. Since $U_{\delta-2} \neq U_{\delta}$ by the minimality of $b$, we have $G_{\delta-1}=$ $\left\langle G_{\delta-2, \delta-1}, G_{\delta-1, \delta}\right\rangle$ by (4) and $(3.10)-(2)$, so $G_{\delta-1}=\left\langle T_{\alpha}, G_{\delta-1, \delta}\right\rangle$. Therefore $V_{\delta}$ $\subseteq Z\left(G_{\delta-1}\right)$ because $V_{\delta} \subseteq U_{\beta} \subseteq T_{\alpha}$ by $(6.5)-(1)$, a contradiction.

(8.3) Let $\xi \in \mathcal{A}$ and $\eta \in \mathcal{B}$. Then the following holds.

(1) $G_{\xi} / C_{G_{\xi}}\left(V_{\xi}\right) \cong \mathbf{D}_{6}$ and $\left|V_{\xi}\right|=2^{2}$.

(2) $\left(G_{\eta}, U_{\eta} / V_{\eta}\right) \ll\left(O_{4}^{+}(2), G F(2)^{4}\right), G_{\eta} / C_{G_{\eta}}\left(U_{\eta} / V_{\eta}\right) \cong O_{4}^{+}(2)$, and $n\left(G_{\eta}, U_{\eta} / V_{\eta}\right)=1$.

Proof. We may assume $\langle\xi, \eta\rangle \in E(\Gamma)$. Then (2) follows from (8.1)-(2), (8.2), and (2.5)-(1). Hence $\left|V_{\xi}: V_{\eta}\right| \leqq 2$ by (2.5)-(3) and (2.15), and so (1) holds by (6.2).

Notation. Let $\langle\xi, \eta\rangle \in E(\Gamma)$.

$$
\begin{aligned}
& W_{\xi}=\cap U_{\eta}^{G_{\xi}} . \\
& T_{\xi}=\left\langle U_{\eta}^{G_{\xi}}\right\rangle . \\
& T_{\xi}^{(n)}=\left[T_{\xi}, Q_{\xi} ; n\right] V_{\xi} \text { for } n \geqq 0 . \\
& K_{\eta}=\left\langle T_{\xi}^{G_{\eta}}\right\rangle . \\
& Z_{\xi \eta}=Z\left(G_{\xi \eta} \bmod Q_{\eta}\right) . \\
& E_{\xi \eta}=C_{G_{\xi \eta}}\left(C_{U_{\eta} \bmod V_{\eta}}\left(Z_{\xi \eta}\right)\right) .
\end{aligned}
$$

(8.4) Let $\xi \in \mathcal{A}$ and $\eta \in \mathcal{B}$.

(1) One of the following holds.

(i) $\left|W_{\xi}\right|=2^{3},\left|U_{\eta}\right|=2^{5}, W_{\xi}=T_{\xi}^{(2)} \neq V_{\xi}, n\left(G_{\xi}, T_{\xi} / W_{\xi}\right) \geqq 2$.

(ii) $\left|W_{\xi}\right|=2^{2},\left|U_{\eta}\right|=2^{5}, W_{\xi}=T_{\xi}^{(3)}=V_{\xi}, n\left(G_{\xi}, T_{\xi} / W_{\xi}\right) \geqq 3$.

(iii) $\left|W_{\xi}\right|=2^{2},\left|U_{\eta}\right|=2^{6}, W_{\xi}=T_{\xi}^{(4)}=V_{\xi}, n\left(G_{\xi}, T_{\xi} / W_{\xi}\right) \geqq 4$.

(2) Let $M=\left[U_{\eta}, O^{2}\left(G_{\eta}\right)\right] V_{\eta}$ and $L=C_{U_{\eta}}\left(O^{2}\left(G_{\eta}\right)\right)$. Then

$$
U_{\eta} / V_{\eta}=M / V_{\eta} \times L / V_{\eta} \text { and }\left|L / V_{\eta}\right| \leqq 2 .
$$

Proof. We may assume $\langle\xi, \eta\rangle \in E(\Gamma)$. By (3.9), we have

$$
\begin{aligned}
& 2^{5} \leqq\left|U_{\eta}\right| \leqq 2^{6},\left|W_{\xi}: V_{\xi}\right| \leqq 2, \quad \text { and } \\
& T_{\xi}^{(m-1)} \neq W_{\xi}=T_{\xi}^{(m)} \text { if }\left|U_{\eta}: W_{\xi}\right|=2^{m} .
\end{aligned}
$$


Therefore, to prove (1), we will assume $\left|U_{\eta}\right|=2^{6}$ and $\left|W_{\xi}\right|=2^{3}$, and argue for a contradiction. Let $L=C_{U_{\eta}}\left(O^{2}\left(G_{\eta}\right)\right)$ and $D=C_{Q_{\eta}}(L)$. As $|L|=2^{2},\left|Q_{\eta}: D\right| \leqq 2$, so $D \nsubseteq Q_{\xi}$ by $(3.2)-(2)$. Suppose $L \nsubseteq W_{\xi}$. Then, since $\left|U_{\eta}: L W_{\xi}\right|=2^{2}$ and $\left[L W_{\xi}, Q_{\xi}\right] \subseteq L V_{\xi} \cap W_{\xi}=V_{\xi}$, we have $T_{\xi}^{(3)} \subseteq V_{\xi} \subset W_{\xi}$, a contradiction. Thus $L \subseteq W_{\xi}$, and so $\left|W_{\xi}: L\right|=2$. Now, take $x \in G_{\xi}$ so that $G_{\xi}=\left\langle G_{\xi \eta}, x\right\rangle$. Then $L \cap L^{x} \neq 1$. Since $\left\langle D, D^{x}\right\rangle$ contains a Sylow 3-subgroup of $G_{\xi}, L \cap L^{x}$ is centralized both by a Sylow 3-subgroup of $G_{\xi}$ and by a Sylow 3-subgroup of $G_{\eta}$. Therefore $L \cap L^{x}=1$ by (3.1), a contradiction. Thus (1) holds. Part (2) is a consequence of (6.3), (2.4)-(3), (8.3)-(2), and (1).

(8.5) Let $\langle\xi, \eta\rangle \in E(\Gamma)$, and suppose $\left|W_{\xi}\right|=2^{3}$. Then

$$
C_{G_{\xi \eta}}\left(W_{\xi}\right) \subseteq Q_{\xi} \cap E_{\xi \eta},\left[T_{\xi}, C_{G_{\xi \eta}}\left(W_{\xi}\right)\right] \subseteq W_{\xi} \text {, and }\left[K_{\eta}, C_{G_{\xi \eta}}\left(U_{\eta}\right)\right] \subseteq U_{\eta} \text {. }
$$

Proof. We have $E_{\xi \eta}=C_{G_{\xi \eta}}\left(W_{\xi} / V_{\eta}\right) \supseteqq C_{G_{\xi \eta}}\left(W_{\xi}\right) \cong C_{G_{\xi \eta}}\left(V_{\xi}\right)=Q_{\xi}$, $\left[U_{\eta}, E_{\xi \eta}\right]=W_{\xi}$ and $C_{G_{\xi \eta}}\left(U_{\eta}\right) \subseteq C_{G_{\xi \eta}}\left(U_{\eta} / V_{\eta}\right)=Q_{\eta}$ by $(2.5)-(3)(4),(2.4)-(1)$, and (6.3), and so $\left[U_{\eta}, C_{Q_{\xi}}\left(W_{\xi}\right)\right] \subseteq W_{\xi}$. Therefore $\left[T_{\xi}, C_{Q_{\eta}}\left(U_{\eta}\right)\right] \subseteq\left[T_{\xi}, C_{G_{\xi \eta}}\left(W_{\xi}\right)\right]=$ $\left[T_{\xi}, C_{Q_{\xi}}\left(W_{\xi}\right)\right]=\left\langle\left[U_{\eta}, C_{Q_{\xi}}\left(W_{\xi}\right)\right]^{G_{\xi}}\right\rangle \subseteq W_{\xi} \subseteq U_{\eta}$, and hence $\left[K_{\eta}, C_{G_{\xi \eta}}\left(U_{\eta}\right)\right]=$ $\left[K_{\eta}, C_{Q_{\eta}}\left(U_{\eta}\right)\right]=\left\langle\left[T_{\xi}, C_{Q_{\eta}}\left(U_{\eta}\right)\right]^{G_{\eta}}\right\rangle \subseteq U_{\eta}$.

(8.6) Let $\beta, \delta \in \mathcal{B}$ and $d(\beta, \delta)=b-1$. Let $\alpha \in \Delta(\beta)$ and $\theta \in \Delta(\alpha)$, and assume

$$
U_{\delta} \cap Q_{\beta} \nsubseteq Q_{\alpha} \quad \text { and } \quad G_{\alpha}=\left\langle G_{\theta \alpha}, G_{\alpha \beta}\right\rangle .
$$

Then $C_{U_{\theta}}\left(U_{\delta}\right) \subseteq W_{\alpha}$.

Proof. Let $F=C_{U_{\theta}}\left(U_{\delta}\right)$. Note that $G_{\alpha}=\left\langle Q_{\theta}, U_{\delta} \cap Q_{\beta}\right\rangle$ by (3.2)-(3). Since $V_{\alpha} F$ is normalized by $Q_{\theta}$ and $U_{\delta} \cap Q_{\beta}$, we have $V_{\alpha} F \triangleleft G_{\alpha}$, and hence $F \subseteq V_{\alpha} F$ $\subseteq \cap U_{\theta} G_{\alpha}=W_{\alpha}$.

(8.7) Let $\beta, \delta \in \mathcal{B}$ and $d(\beta, \delta)=b-1$, and $\alpha \in \Delta(\beta)$. Suppose $\left|W_{\alpha}\right|=2^{3}$ and $\left[V_{\alpha}, T_{\delta-1}\right] \neq 1$, and define $B=\left[T_{\alpha}, U_{\delta-2}\right]$. Then the following holds.

(1) $\quad B \subseteq W_{\alpha} \cap W_{\delta-3}$ and $\left|V_{\beta} B\right| \leqq 2^{2}$.

(2) Assume $Z_{\delta-3, \delta-2} \subseteq T_{\alpha} Q_{\delta-2}$. Then $C_{U_{\delta-2}}\left(T_{\alpha}\right)=W_{\delta-3}, B \cap V_{\delta-2}=1$, $V_{\beta} \subseteq B$, and $\left|W_{\alpha} \cap W_{\delta-3}\right| \geqq 2^{2}$.

(3) Assume $V_{\beta} \nsupseteq B \nsubseteq V_{\delta-2}$ and $\left|K_{\delta-2}: K_{\delta-2} \cap G_{\alpha \beta}\right| \leqq 2$. Then

$$
\left|K_{\delta-2}: K_{\delta-2} \cap Q_{\alpha}\right| \cdot\left|\left[T_{\alpha}, K_{\delta-2} \cap Q_{\alpha}\right] U_{\delta-2}: U_{\delta-2}\right| \leqq 2^{a}
$$

where $a=\max \{2,8-b\}$.

Proof. Since $\left[W_{\alpha}, U_{\delta-2}\right] \subseteq\left[U_{\beta}, U_{\delta-2}\right]=1$ and $\left[T_{\alpha}, W_{\delta-3}\right] \subseteq\left[T_{\alpha}, U_{\delta-4}\right]=$ 1 by the minimality of $b$, we have $B \subseteq W_{\alpha} \cap W_{\delta-3}$ and $T_{\alpha} \subseteq E_{\delta-3, \delta-2}$ by (8.5). Also, since $V_{\beta} B \subseteq C_{W_{\alpha}}\left(T_{\delta-1}\right) \nsupseteq V_{\alpha}$, we have $\left|V_{\beta} B\right| \leqq 2^{2}$. 
Suppose $Z_{\delta-3, \delta-2} \subseteq T_{\alpha} Q_{\delta-2}$. Then we have $C_{U_{\delta-2}}\left(T_{\alpha}\right)=W_{\delta-3}=B V_{\delta-2}$ by (2.5)-(4). Therefore $B \cap V_{\delta-2}=1$ and $\left|W_{\alpha} \cap W_{\delta-3}\right| \geqq|B|=2^{2}$, and hence $V_{\beta} \subseteq$ $B$.

Now, suppose $V_{\beta} \nsupseteq B \nsubseteq V_{\delta-2}$ and $\left|K_{\delta-2}: K_{\delta-2} \cap G_{\alpha \beta}\right| \leqq 2$. Then $C_{W_{\alpha}}\left(T_{\delta-1}\right)$ $=V_{\beta} B$ and $\left|W_{\alpha}: V_{\beta} B\right|=2$, so $W_{\alpha}=V_{\alpha} B$. Since $\left[B, K_{\delta-2}\right] \subseteq\left[U_{\delta-2}, K_{\delta-2}\right]=1$ by the minimality of $b$, we have $\left[W_{\alpha}, K_{\delta-2} \cap Q_{\alpha}\right]=\left[V_{\beta} B, K_{\delta-2} \cap Q_{\alpha}\right]=1$. Hence $\left[T_{\alpha}, K_{\delta-2} \cap Q_{\alpha}\right] \subseteq W_{\alpha} \cap K_{\delta-2}$ by (8.5), and, in particular, $\left[T_{\alpha}, K_{\delta-2} \cap Q_{\alpha}\right] \subseteq$ $V_{\beta} B$ if $b \geqq 7$ as $K_{\delta-2}$ is abelian in that case. Note that, if $K_{\delta-2} \cap G_{\alpha \beta} \nsubseteq Q_{\alpha}$, $V_{\beta}=\left[V_{\alpha}, K_{\delta-2} \cap G_{\alpha \beta}\right] \subseteq\left[U_{\beta}, K_{\delta-2}\right] \subseteq U_{\delta-2}$ by (8.5). Therefore, we have

$$
\left|K_{\delta-2}: K_{\delta-2} \cap Q_{\alpha}\right| \cdot\left|\left[T_{\alpha}, K_{\delta-2} \cap Q_{\alpha}\right] U_{\delta-2}: U_{\delta-2}\right| \leqq 2^{a}
$$

both in the case $K_{\delta-2} \cap G_{\alpha \beta} \subseteq Q_{\alpha}$ and in the case $K_{\delta-2} \cap G_{\alpha \beta} \nsubseteq Q_{\alpha}$.

(8.8) Let $\alpha \in \mathcal{A}$. Suppose $b=5$ and $\left|W_{\alpha}\right|=2^{3}$. Take $\theta, \beta \in \Delta(\alpha)$ so that $G_{\alpha}=\left\langle G_{\theta \alpha}, G_{\alpha \beta}\right\rangle$. Then the following holds.

(1) $K_{\beta} Q_{\theta}=Q_{\theta}, Z_{\theta \alpha}$, or $E_{\theta \alpha}$.

(2) If $K_{\beta} Q_{\theta}=E_{\theta \alpha}$, then $n\left(G_{\beta}, K_{\beta} / U_{\beta}\right) \geqq 2$.

Proof. Since $\left[W_{\alpha}, K_{\beta}\right] \subseteq\left[U_{\beta}, K_{\beta}\right]=1$, we have $K_{\beta} \subseteq E_{\theta \alpha}$ by (8.5). Also, since $G_{\alpha}=\left\langle G_{\theta \alpha}, G_{\alpha \beta}\right\rangle$, we have $G_{\theta \alpha}=Q_{\theta}\left(Q_{\alpha} \cap Q_{\beta}\right)$ by (3.2)-(3), and so $K_{\beta} Q_{\theta} \triangleleft G_{\theta \alpha}$. Therefore $K_{\beta} Q_{\theta}=Q_{\theta}, Z_{\theta \alpha}$, or $E_{\theta \alpha}$ because $G_{\theta \alpha} / Q_{\theta} \cong \mathbf{D}_{8}$.

Suppose $K_{\beta} Q_{\theta}=E_{\theta \alpha}$. Then $\left[K_{\beta}, G_{\theta \alpha}\right] Q_{\theta}=\left[K_{\beta}, Q_{\beta} \cap Q_{\alpha}\right] Q_{\theta} \neq Q_{\theta}$, and so $\left[K_{\beta}, Q_{\beta}\right] \nsubseteq T_{\alpha}$. Thus $n\left(G_{\beta}, K_{\beta} / U_{\beta}\right) \geqq 2$ by $(2.14)-(2)$. holds.

(8.9) Let $\beta \in \mathcal{B}$ and $\alpha, \xi \in \Delta(\beta)$, and suppose $\left|W_{\alpha}\right|=2^{3}$. Then the following

(1) If $W_{\alpha} \cap W_{\xi}=V_{\beta}$ (or, equivalently, $U_{\beta}=W_{\alpha} W_{\xi}$ ), then $G_{\beta}=\left\langle G_{\alpha \beta}, x\right\rangle$ $=\left\langle Q_{\alpha}, x\right\rangle$ for all $x \in E_{\beta \xi}-Q_{\beta}$.

(2) If $W_{\alpha} \neq W_{\alpha} \cap W_{\xi} \neq V_{\beta}$ (or, equivalently, $W_{\alpha} \neq W_{\alpha} W_{\xi} \neq U_{\beta}$ ), then $W_{\alpha}=V_{\alpha}\left(W_{\alpha} \cap W_{\xi}\right)$ and $G_{\alpha \beta} \cap G_{\beta \xi}=E_{\alpha \beta} \cap E_{\beta \xi}$.

Proof. Put $D_{\beta}=C_{G_{\beta}}\left(U_{\beta} / V_{\beta}\right)$. Then $D_{\beta} \cap G_{\beta \xi}=Q_{\beta}$ by (6.3) and (2.4)-(1).

Suppose first that $W_{\alpha} \cap W_{\xi}=V_{\beta}$, and let $x \in E_{\beta \xi}-Q_{\beta}$. Then $G_{\alpha \beta} D_{\beta} \cap E_{\beta \xi}=$ $E_{\alpha \beta} D_{\beta} \cap E_{\beta \xi}=D_{\beta} \cap G_{\beta \xi}=Q_{\beta}$ by $(2.5)-(12)$, so $x \notin G_{\alpha \beta} D_{\beta}$. Thus $G_{\beta}=\left\langle G_{\alpha \beta}, x\right\rangle$ because $G_{\alpha \beta} D_{\beta}$ is the unique maximal subgroup of $G_{\beta}$ containing $G_{\alpha \beta}$, and hence $G_{\beta}=\left\langle Q_{\alpha}, x\right\rangle$ by $(3.2)-(3)$.

Suppose next that $W_{\alpha} \neq W_{\alpha} \cap W_{\xi} \neq V_{\beta}$. Since $W_{\alpha} \neq W_{\xi}$, we have $G_{\beta}=$ $\left\langle G_{\alpha \beta}, G_{\beta \xi}\right\rangle$ by (8.2) and (3.10)-(2), and hence $G_{\beta}=\left\langle G_{\alpha \beta}, Z_{\beta \xi}\right\rangle$ by (2.1)-(10). Thus $V_{\alpha} \nsubseteq C_{U_{\beta} \bmod V_{\beta}}\left(Z_{\beta \xi}\right)=W_{\xi}$ by $(2.5)-(4)$, and so $W_{\alpha}=V_{\alpha}\left(W_{\alpha} \cap W_{\xi}\right)$. Put $R=E_{\alpha \beta} D_{\beta} \cap E_{\beta \xi}$. Then $R \nsubseteq Q_{\beta}$ by (2.5)-(11). If $G_{\alpha \beta} \cap G_{\beta \xi} \nsubseteq R$, then $Z_{\beta \xi} \subseteq$ $\left\langle G_{\alpha \beta} \cap G_{\beta \xi}, R\right\rangle \cong G_{\alpha \beta}$ because $G_{\beta \xi} / Q_{\beta} \cong \mathbf{D}_{8}$, a contradiction. Hence we have $G_{\alpha \beta} \cap G_{\beta \xi} \subseteq R \cong E_{\beta \xi}$, and so, by symmetry, $G_{\alpha \beta} \cap G_{\beta \xi} \subseteq E_{\alpha \beta} \cap E_{\beta \xi}$. 
Proposition 8.10 Let $\beta, \delta \in \mathcal{B}$ and $d(\beta, \delta)=b-1$. Then

$$
\left[U_{\beta}, U_{\delta} \cap Q_{\beta}\right]=1 \text {. }
$$

Proof. Suppose false. Then $\left[U_{\beta}, U_{\delta} \cap Q_{\beta}\right]=V_{\beta}$. Define $D_{\eta}=C_{G_{\eta}}\left(U_{\eta} / V_{\eta}\right)$ for all $\eta \in \mathcal{B}$.

(1) There exists $\alpha \in \Delta(\beta)$ such that

$$
\left[V_{\alpha}, U_{\delta} \cap Q_{\beta}\right]=V_{\beta} \quad \text { and } \quad G_{\alpha \beta} \cap G_{\beta, \beta+1} \neq Q_{\beta} .
$$

Proof. Choose $\alpha \in \Delta(\beta)$ so that $\left[V_{\alpha}, U_{\delta} \cap Q_{\beta}\right]=V_{\beta}$, and put $R=G_{\alpha \beta} D_{\beta} \cap$ $G_{\beta, \beta+1}$. Then $R \neq Q_{\beta}$ by (8.3)-(2) and (2.5)-(12). Since $G_{\beta} \neq\left\langle G_{\alpha \beta}, R\right\rangle$, there exists $x \in G_{\beta}$ such that $R \subseteq G_{\alpha \beta}{ }^{x}$ and $V_{\alpha^{x}}=V_{\alpha}$ by (8.2) and (3.10)-(1). Thus $\alpha^{x}$ has the desired properties.

Now, let $\alpha \in \Delta(\beta)$ be as in (1). Define

$$
B=\left[T_{\alpha}, U_{\delta-2}\right] \text { and } D=\left[T_{\alpha} \cap Q_{\delta-1}, U_{\delta} \cap Q_{\beta}\right] .
$$

(2) The following holds.

(i) $U_{\delta} \cap Q_{\beta} \nsubseteq Q_{\alpha}$.

(ii) $V_{\beta} \subseteq D \leqq T_{\alpha} \cap\left[T_{\alpha} \cap Q_{\delta-1}, U_{\delta}\right] \subseteq T_{\alpha} \cap U_{\delta}$.

(iii) $|D| \leqq 2^{3}$ and $\left|W_{\alpha} D: W_{\alpha}\right| \leqq\left|D: V_{\beta}\right| \leqq 2^{2}$.

(iv) If $|D|=2^{3}$ and $\left|U_{\delta}\right|=2^{5}$, then $D \supseteqq V_{\delta-1}$.

Proof. Parts (i) and (ii) follow from the choice of $\alpha$ and the definition of $D$. Now, suppose $\left|U_{\delta}: D\right| \leqq 2^{2}$. Then $T_{\alpha} \cap Q_{\delta-1}$ induces a quasitransvection on $U_{\delta} / V_{\delta}$, and so $|D| \leqq\left|\left[T_{\alpha} \cap Q_{\delta-1}, U_{\delta}\right] V_{\delta}\right|=\left|\left[T_{\alpha} \cap Q_{\delta-1}, M\right] V_{\delta}\right| \leqq 2^{3}$ by $(2.5)-(10)$ and (8.4)-(2), where $M=\left[U_{\delta}, O^{2}\left(G_{\delta}\right)\right] V_{\delta}$. Thus, since $\left|U_{\delta}\right| \leqq 2^{6}$, we have $|D| \leqq 2^{3}$, and so $\left|W_{\alpha} D: W_{\alpha}\right| \leqq\left|D: V_{\beta}\right| \leqq 2^{2}$. Finally, let $|D|=2^{3}$ and $\left|U_{\delta}\right|=2^{5}$. Then $D=\left[T_{\alpha} \cap Q_{\delta-1}, U_{\delta}\right] V_{\delta} \supseteqq V_{\delta-1}$ by the above remark and (2.5)-(10).

Now, let $n=n\left(G_{\xi}, T_{\xi} / W_{\xi}\right)$ for $\xi \in \mathcal{A}$. Note that $n \geqq 2$ by $(8.4)$.

$$
O^{2}\left(G_{\xi} / C_{G_{\xi}}\left(T_{\xi} / W_{\xi}\right)\right) \cong \mathbf{Z}_{3} \text { for } \xi \in \mathcal{A} \text {. }
$$

Proof. We may assume $\xi=\alpha$, and it is sufficient to prove that $U_{\delta} \cap Q_{\beta}$ induces a factorwise quasitransvection on $T_{\alpha} / W_{\alpha}$ as in the proof of (4) of (8.2).

Since $T_{\alpha} \subseteq Q_{\delta-3}$ by the minimality of $b$,

$$
\begin{aligned}
& \left|T_{\alpha}: T_{\alpha} \cap Q_{\delta-1}\right| \cdot\left|W_{\alpha} D: W_{\alpha}\right| \\
& \quad \leqq\left|T_{\alpha}: T_{\alpha} \cap Q_{\delta-2}\right| \cdot\left|T_{\alpha} \cap Q_{\delta-2}: T_{\alpha} \cap Q_{\delta-2} \cap Q_{\delta-1}\right| \cdot\left|D: V_{\beta}\right| \\
& \quad \leqq 2^{2} \cdot 2 \cdot 2^{2}
\end{aligned}
$$


by (2-iii), so we may assume $n \leqq 3$, and hence $\left|U_{\beta}\right|=2^{5}$ by (8.4). If $|D|=2^{3}$, then $V_{\delta-1} \subseteq D \subseteq T_{\alpha}$ by (2-ii,iv), so $T_{\alpha} \cap Q_{\delta-2} \subseteq C_{G_{\delta-2, \delta-1}}\left(V_{\delta-1}\right)=Q_{\delta-1}$. Hence we have either

$$
\left|W_{\alpha} D: W_{\alpha}\right| \leqq\left|D: V_{\beta}\right| \leqq 2 \text { or } \quad T_{\alpha} \cap Q_{\delta-2} \leqq Q_{\delta-1}
$$

Therefore

$$
\left|T_{\alpha}: T_{\alpha} \cap Q_{\delta-1}\right| \cdot\left|W_{\alpha} D: W_{\alpha}\right| \leqq 2^{4},
$$

so we may assume $n=2$, and then $\left|W_{\alpha}\right|=2^{3}$ by (8.4).

Now, suppose

$$
\left|T_{\alpha}: T_{\alpha} \cap Q_{\delta-1}\right| \cdot\left|W_{\alpha} D: W_{\alpha}\right|=2^{4} .
$$

Then $\left|T_{\alpha}: T_{\alpha} \cap Q_{\delta-2}\right|=2^{2} \leqq\left|T_{\alpha}: T_{\alpha} \cap Q_{\delta-1}\right|$ by $(*)$, and hence $C_{U_{\delta-2}}\left(T_{\alpha}\right)=W_{\delta-3}$, $B \cap V_{\delta-2}=1$, and $\left|W_{\alpha} \cap W_{\delta-3}\right| \geqq 2^{2}$ by (8.7)-(2). Since $\left[T_{\alpha} \cap Q_{\delta-2}, W_{\delta-1}\right] \subseteq\left[T_{\alpha} \cap\right.$ $\left.Q_{\delta-2}, U_{\delta-2}\right] \subseteq B \cap V_{\delta-2}=1$, we have $T_{\alpha} \cap Q_{\delta-2} \subseteq Q_{\delta-1}$, so $T_{\alpha} \cap Q_{\delta-1}=T_{\alpha} \cap Q_{\delta-2}$. Thus $D \subseteq\left[T_{\alpha} \cap Q_{\delta-1}, U_{\delta}\right] \subseteq W_{\delta-1} \subseteq U_{\delta-2}$, and so $D \subseteq C_{U_{\delta-2}}\left(T_{\alpha}\right)=W_{\delta-3}$. Therefore $\left|W_{\alpha} D: W_{\alpha}\right| \leqq\left|W_{\alpha} W_{\delta-3}: W_{\alpha}\right| \leqq 2$, and hence

$$
\left|T_{\alpha}: T_{\alpha} \cap Q_{\delta-1}\right| \cdot\left|W_{\alpha} D: W_{\alpha}\right| \leqq 2^{3},
$$

which is a contradiction as required.

(4) $T_{\alpha} \nsubseteq Q_{\delta-1}$.

Proof. Suppose false. Since $\left|W_{\alpha} D: W_{\alpha}\right| \leqq 2^{2}$ by (2-iii), we have $n=2$ by (2.11)-(2). Therefore

by (8.4), and so

(i) $\left|W_{\alpha}\right|=2^{3}$ and $\left|U_{\beta}\right|=2^{5}$

(ii) $|D|=2^{3}$ and $W_{\alpha} \cap D=V_{\beta}$.

Here, we will prove that

(iii) $\left[T_{\alpha}, U_{\delta}\right] \subseteq D$ and $\left|U_{\delta}: U_{\delta} \cap Q_{\alpha}\right|=2$.

To show this, we may assume $U_{\delta} \nsubseteq Q_{\beta}$. As $\left|U_{\delta} \cap Q_{\beta}: D\right| \leqq 2$ and $\left|D: V_{\delta}\right|=2^{2}$ by (i) and (ii), $\left|T_{\alpha}: T_{\alpha} \cap Q_{\delta}\right|=2^{2}=\left|U_{\delta}: D\right| \geqq\left|U_{\delta}: C_{U_{\delta}}\left(T_{\alpha}\right)\right|$, so $T_{\alpha} Q_{\delta}=$ $E_{\delta-1, \delta}$ and $D=W_{\delta-1}$ by $(2.5)-(4)(5)$. Therefore, $\left[T_{\alpha}, U_{\delta}\right] \subseteq W_{\delta-1}=D \subseteq T_{\alpha}$ by (2.5)-(4) and (2-ii), and hence $G_{\beta} \neq\left\langle G_{\alpha \beta}, U_{\delta}\right\rangle$. If $U_{\delta} \varsubsetneqq G_{\alpha \beta}$, then $Z_{\beta, \beta+1} \leqq$ $\left\langle G_{\alpha \beta} \cap G_{\beta, \beta+1}, U_{\delta}\right\rangle$, so $G_{\beta}=\left\langle G_{\alpha \beta}, Z_{\beta, \beta+1}\right\rangle=\left\langle G_{\alpha \beta}, U_{\delta}\right\rangle$, a contradiction. Thus we have $U_{\delta} \subseteq G_{\alpha \beta}$, and hence $\left|U_{\delta}: U_{\delta} \cap Q_{\alpha}\right| \leqq\left|G_{\alpha \beta}: Q_{\alpha}\right|=2$, proving (iii).

Now, take $\theta \in \Delta(\alpha)$ so that $G_{\alpha}=\left\langle G_{\theta \alpha}, G_{\alpha \beta}\right\rangle$. Then $G_{\alpha}=\left\langle G_{\theta \alpha}, U_{\delta} \cap Q_{\beta}\right\rangle=$ $\left\langle Q_{\theta}, U_{\delta} \cap Q_{\beta}\right\rangle$ by $(2-\mathrm{i})$ and $(3.2)-(3)$. Since $\left[U_{\theta}, U_{\delta} \cap Q_{\alpha}\right] \subseteq C_{U_{\theta}}\left(U_{\delta}\right) \subseteq W_{\alpha}$ by (8.6), we have $\left[U_{\theta}, U_{\delta} \cap Q_{\alpha}\right] \subseteq W_{\alpha} \cap D=V_{\beta} \subseteq V_{\alpha}$ by (ii) and (iii), so $U_{\delta} \cap Q_{\alpha}$ $\subseteq Q_{\theta}$ by $(2.5)-(9)$. Therefore $\left[U_{\theta}, U_{\delta} \cap Q_{\alpha}\right] \subseteq C_{V_{\theta}}\left(U_{\delta}\right)=1$ by the choice of $\theta$, and hence $U_{\theta}$ induces a transvection on $U_{\delta} / V_{\delta}$ by (iii). Thus $\left|\left[U_{\theta}, U_{\delta}\right]\right| \leqq 2^{2}$, and so 
$\left|U_{\theta}\left[U_{\theta}, U_{\delta}\right]: U_{\theta}\right| \leqq\left|\left[U_{\theta}, U_{\delta}\right]: V_{\beta}\right| \leqq 2$ as $V_{\beta}=\left[V_{\alpha}, U_{\delta} \cap Q_{\beta}\right] \leqq\left[U_{\theta}, U_{\delta}\right]$. Now, take $x \in U_{\delta} \cap Q_{\beta}-Q_{\alpha}$. Then $\cap U_{\theta} G_{\alpha}=W_{\alpha} \subset U_{\theta} \cap U_{\theta}{ }^{x} \triangleleft\left\langle Q_{\theta}, x\right\rangle=G_{\alpha}$, a contradiction.

(5) $\quad T_{\alpha} \nsubseteq Q_{\delta-2},\left|W_{\alpha}\right|=2^{3},\left|U_{\beta}\right|=2^{5}, W_{\delta-1} \supseteqq V_{\beta} \nsupseteq B \nsubseteq V_{\delta-2}$, and $\left|K_{\delta-2}: K_{\delta-2} \cap G_{\alpha \beta}\right| \leqq 2$.

Proof. Take $\theta \in \Delta(\alpha)$ so that $U_{\theta} \nsubseteq Q_{\delta-1}$. Then $U_{\theta} \neq U_{\beta}$, so $G_{\alpha}=$ $\left\langle G_{\theta \alpha}, G_{\alpha \beta}\right\rangle$ by (3) together with (3.10)-(2), and then $V_{\theta} \neq V_{\beta}$ by (8.3)-(1) and (3.10)-(2). Let $F=\left[U_{\theta}, U_{\delta-2}\right]$. Then $B \supseteqq F \cong C_{U_{\theta}}\left(U_{\delta}\right) \subseteq W_{\alpha}$ by (8.6). Suppose $F \subseteq V_{\alpha}$. Then $U_{\delta-2} \subseteq Q_{\theta}$ by $(2.5)-(9)$, and so $F \cong C_{V_{\theta}}\left(U_{\delta}\right)=1$. Thus $U_{\theta}$ $\subseteq C_{G_{\delta-3, \delta-2}}\left(U_{\delta-2}\right) \subseteq C_{Q_{\delta-2}}\left(V_{\delta-1}\right) \subseteq Q_{\delta-1}$ by (8.5), a contradiction. Therefore $F \nsubseteq V_{\alpha}$, so

(i) $\quad B \nsubseteq V_{\beta} \subseteq V_{\alpha} \neq V_{\alpha} F \cong W_{\alpha}$.

Hence we have

(ii) $\quad\left|W_{\alpha}\right|=2^{3}$ and $\left|U_{\beta}\right|=2^{5}$

by (8.4), and then, as $\left[U_{\beta}, W_{\delta-1}\right] \subseteq\left[U_{\beta}, U_{\delta-2}\right]=1$ by the minimality of $b, V_{\beta} \subseteq$ $\left[U_{\beta}, U_{\delta}\right] \subseteq W_{\delta-1}$ by $(8.5)$.

If $W_{\alpha} \cap W_{\beta+1} \neq V_{\beta}$, then $E_{\alpha \beta} \cap E_{\beta, \beta+1} \neq Q_{\beta}$ by the choice of $\alpha$ and (8.9)-(2), and so

$$
\left|K_{\delta-2}: K_{\delta-2} \cap G_{\alpha \beta}\right| \leqq\left|E_{\beta, \beta+1}: E_{\alpha \beta} \cap E_{\beta, \beta+1}\right| \leqq 2 .
$$

If $W_{\alpha} \cap W_{\beta+1}=V_{\beta}$, then $B \nsubseteq W_{\beta+1}$ as $B \subseteq W_{\alpha}$ by $(8.7)-(1)$, so $K_{\delta-2}$ centralizes a hyperplane $B W_{\beta+1}$ of $U_{\beta}$. Thus

$$
\left|K_{\delta-2}: K_{\delta-2} \cap G_{\alpha \beta}\right| \leqq\left|K_{\delta-2}: K_{\delta-2} \cap Q_{\beta}\right| \leqq 2
$$

by $(2.5)-(5)$.

Finally, suppose $T_{\alpha} \subseteq Q_{\delta-2}$. If $|D|=2^{3}$, then $V_{\delta-1} \subseteq D \subseteq T_{\alpha}$ by (2-ii,iv), and so $T_{\alpha} \leqq C_{Q_{\delta-2}}\left(V_{\delta-1}\right)=Q_{\delta-1}$, which contradicts (4). Therefore $|D| \leqq 2^{2}$, and hence $\left|T_{\alpha}: T_{\alpha} \cap Q_{\delta-1}\right| \cdot\left|W_{\alpha} D: W_{\alpha}\right| \leqq 2 \cdot 2=2^{2}$. Thus we have $n=2$ by (8.4), and so

(iii) $|D|=2^{2}$ and $D \nsubseteq W_{\alpha}$.

Here, we will prove that

(iv) $D \supseteqq V_{\delta-2}$.

Note that $D \supseteqq\left[T_{\alpha} \cap Q_{\delta-1}, W_{\delta-1}\right] \subseteq\left[T_{\alpha}, U_{\delta-2}\right] \subseteq V_{\delta-2}$. Thus we may assume that $\left[T_{\alpha} \cap Q_{\delta-1}, W_{\delta-1}\right]=1$, so $D \subseteq\left[T_{\alpha} \cap Q_{\delta-1}, U_{\delta}\right] \subseteq W_{\delta-1}$ by (8.5). Therefore $D \cap V_{\delta-1} \neq 1$ by (ii) and (iii), and hence $D \cap V_{\delta-1}=C_{V_{\delta-1}}\left(T_{\alpha}\right)=V_{\delta-2}$, proving (iv).

Now, since $V_{\delta-2}=F \nsubseteq V_{\alpha}$ by (i), $V_{\beta} \neq V_{\delta-2}$, so $D=V_{\beta} V_{\delta-2}=V_{\beta} F \subseteq W_{\alpha}$ by (iv) and (i), contrary to (iii). Thus $T_{\alpha} \nsubseteq Q_{\delta-2}$, and hence $B \nsubseteq V_{\delta-2}$.

(6) If $T_{\alpha} Q_{\delta-2} \neq Z_{\delta-3, \delta-2}$, then $b \geqq 7, G_{\delta-2}=\left\langle G_{\delta-3, \delta-2}, G_{\delta-2, \delta-1}\right\rangle$, and $O^{2}\left(G_{\eta} / Q_{\eta} C_{G_{\eta}}\left(K_{\eta}\right)\right) \cong \mathbf{E}_{3^{2}}$ for $\eta \in \mathcal{B}$. 
Proof. We may assume $\eta=\delta-2$. Let $\bar{G}_{\delta-2}=G_{\delta-2} / Q_{\delta-2} C_{G_{\delta-2}}\left(K_{\delta-2} / U_{\delta-2}\right)$. By (5) together with (8.7)-(3), we have

$$
\left|K_{\delta-2}: K_{\delta-2} \cap Q_{\alpha}\right| \cdot\left|\left[T_{\alpha}, K_{\delta-2} \cap Q_{\alpha}\right] U_{\delta-2}: U_{\delta-2}\right| \leqq 2^{a}
$$

where $a=\max \{2,8-b\}$.

Now, suppose $b=5$. As $Q_{\delta-2} \neq K_{\beta} Q_{\delta-2} \neq Z_{\delta-3, \delta-2}, n\left(G_{\beta}, K_{\beta} / U_{\beta}\right) \geqq 2$ by (8.8)-(2). Thus the above inequality shows that $T_{\alpha}$ induces a factorwise sesquitransvection on $K_{\delta-2} / U_{\delta-2}$ by $(2.11)-(2)$, and hence $O^{2}\left(\bar{G}_{\delta-2}\right) \cong \mathbf{E}_{32}$ by (2.11)-(1). Therefore $G_{\delta-2}=\left\langle G_{\delta-3, \delta-2}, G_{\delta-2, \delta-1}\right\rangle$ by $(3.10)-(2)$ because $T_{\delta-3} \neq$ $T_{\delta-1}$ by the minimality of $b$, and so $G_{\delta-2}=\left\langle G_{\delta-3, \delta-2}, Z_{\delta-2, \delta-1}\right\rangle$ by (2.11)-(11). Hence $V_{\delta-3} \triangleleft G_{\delta-2}$ because $\left[W_{\delta-1}, Z_{\delta-2, \delta-1}\right] \subseteq V_{\delta-2} \subseteq V_{\delta-3}=V_{\beta} V_{\delta-2} \subseteq W_{\delta-1}$ by (3) and (5) together with (2.5)--(4), a contradiction. Thus we have $b \geqq 7$ so $a=2$, and hence $T_{\alpha}$ induces a factorwise quasitransvection on $K_{\delta-2} / U_{\delta-2}$. Since $T_{\alpha} Q_{\delta-2} \neq Z_{\delta-3, \delta-2}$ and $O^{2}\left(\bar{G}_{\delta-2}\right)$ is a 3 -group, we have $O^{2}\left(\bar{G}_{\delta-2}\right) \cong \mathbf{E}_{3^{2}}$ by (2.11)-(1). This shows that $D_{\delta-2}=Q_{\delta-2} C_{G_{\delta-2}}\left(K_{\delta-2} / U_{\delta-2}\right)$, and hence $D_{\delta-2}=$ $Q_{\delta-2} C_{G_{\delta-2}}\left(K_{\delta-2}\right)$.

(7) We have $b \geqq 7, G_{\delta-2}=\left\langle G_{\delta-3, \delta-2}, G_{\delta-2, \delta-1}\right\rangle,\left|T_{\alpha} Q_{\delta-2}: Q_{\delta-2}\right|=2$, $T_{\alpha} Q_{\delta-2} \neq Z_{\delta-3, \delta-2}$, and $O^{2}\left(G_{\eta} / G_{\eta} C_{G_{\eta}}\left(K_{\eta}\right)\right) \cong \mathbf{E}_{3^{2}}$ for $\eta \in \mathcal{B}$.

ProOF. In view of $(6)$, it suffices to show that $Z_{\delta-3, \delta-2} \nsubseteq T_{\alpha} Q_{\delta-2}$. Suppose $Z_{\delta-3, \delta-2} \subseteq T_{\alpha} Q_{\delta-2}$. Then we have $V_{\beta} \subseteq B \subseteq W_{\delta-3}$ and $B \cap V_{\delta-2}=1$ by $(8.7)-(1)(2)$, and so

(i) $\quad W_{\delta-3} \cap W_{\delta-1} \supseteqq V_{\beta} V_{\delta-2} \neq V_{\delta-2}$.

Take $\theta \in \Delta(\alpha)$ so that $U_{\theta} \nsubseteq Q_{\delta-2}$. Then $U_{\theta} \neq U_{\beta}$, so $G_{\alpha}=\left\langle G_{\theta \alpha}, G_{\alpha \beta}\right\rangle$ by (3) and (3.10)-(2), and, moreover, $T_{\alpha}=\left\langle U_{\theta}, U_{\theta^{\prime}}, U_{\beta}\right\rangle$ where $\theta^{\prime}=\theta^{x}$ for some $x \in$ $U_{\delta} \cap Q_{\beta}-Q_{\alpha}$ by $(2-\mathrm{i})$ and (3). Therefore $B=\left[U_{\theta}, U_{\delta-2}\right]\left[U_{\theta^{\prime}}, U_{\delta-2}\right]$ and $\left[U_{\theta}, U_{\delta-2}\right]=$ $\left[U_{\theta}, U_{\delta-2}\right]^{x}=\left[U_{\theta^{\prime}}, U_{\delta-2}\right]$, so $B=\left[U_{\theta}, U_{\delta-2}\right]$. Hence we have $Z_{\delta-3, \delta-2} \subseteq U_{\theta} Q_{\delta-2}$, and so $C_{U_{\delta-2}}\left(U_{\theta}\right)=W_{\delta-3}$ by $(2.5)-(4)$. As $\left[U_{\theta}, U_{\delta-2} \cap Q_{\theta}\right] \subseteq V_{\theta} \cap B=1, U_{\delta-2} \cap Q_{\theta}$ $\subseteq C_{U_{\delta-2}}\left(U_{\theta}\right)=W_{\delta-3}$. Thus $\left|U_{\delta-2} Q_{\theta}: Q_{\theta}\right|=2^{2}$ and so

(ii) $U_{\delta-2} Q_{\theta}=E_{\theta \alpha}$.

Similarly, since $\left[U_{\theta} \cap Q_{\delta-2}, U_{\delta-2}\right] \subseteq B \cap V_{\delta-2}=1$, we have $U_{\theta} \cap Q_{\delta-2} \subseteq$ $C_{U_{\theta}}\left(U_{\delta-2}\right)=W_{\alpha}$ by (ii), so $\left|U_{\theta}: U_{\theta} \cap Q_{\delta-2}\right|=2^{2}$. Thus $T_{\alpha} Q_{\delta-2} \supseteq U_{\theta} Q_{\delta-2}=$ $E_{\delta-3, \delta-2}$, and hence $O^{2}\left(G_{\delta-2} / Q_{\delta-2} C_{G_{\delta-2}}\left(K_{\delta-2}\right)\right) \cong \mathrm{E}_{3^{2}}$ by $(6)$. Therefore we have $G_{\delta-2}=\left\langle G_{\delta-3, \delta-2}, G_{\delta-2, \delta-1}\right\rangle$ and

(iii) $\quad W_{\delta-3} \cap W_{\delta-1}=V_{\beta} V_{\delta-2} \neq V_{\delta-2}$

by (i) and (3.10)-(2) because $T_{\delta-3} \neq T_{\delta-1}$ by the minimality of $b$.

Now, put $R=U_{\theta} \cap E_{\delta-2, \delta-1} D_{\delta-2}$. Since $R \nsubseteq Q_{\delta-2}$ by (iii) and (2.5)-(11), we have $\left|U_{\theta}: R\right|=\left|R: W_{\alpha}\right|=2$. However, $\left[R, U_{\delta-2}\right] \subseteq B \cap W_{\delta-3} \cap W_{\delta-1}=V_{\beta} \subseteq$ $V_{\alpha}$, which is impossible under the condition (ii) by (2.5)-(8). 
(8) $V_{\beta} \neq V_{\delta-2}$.

Proof. Suppose false. As $U_{\delta-2} \neq U_{\delta}, G_{\delta-1}=\left\langle G_{\delta-2, \delta-1}, G_{\delta-1, \delta}\right\rangle$ by (3) and $(3.10)-(2)$, so

$$
\left[U_{\beta}, U_{\delta} \cap Q_{\beta}\right]=V_{\beta}=V_{\delta-2} \neq V_{\delta} .
$$

Therefore $V_{\delta-1} \supseteqq\left[U_{\beta}, U_{\delta} \cap Q_{\beta}\right] \varsubsetneqq V_{\delta}$, and hence $U_{\beta} \varsubsetneqq Q_{\delta}$. If $U_{\delta} \subseteq Q_{\beta}$, then $\left[U_{\beta}, U_{\delta}\right]=V_{\delta-2} \subseteq V_{\delta-1}$, so $U_{\beta} \subseteq Q_{\delta}$ by $(2.5)-(9)$, a contradiction. Thus $U_{\delta} \nsubseteq Q_{\beta}$, and hence

$$
U_{\beta} Q_{\delta}=Z_{\delta-1, \delta}
$$

by $(2.5)-(8)$. As $U_{\beta} \neq U_{\beta+2}$, we have $G_{\beta+1}=\left\langle G_{\beta, \beta+1}, G_{\beta+1, \beta+2}\right\rangle$ and $V_{\beta} \neq V_{\beta+2}$ by (3.10)-(2) as above. Therefore,

$$
W_{\beta+1}=V_{\beta+1}\left[U_{\beta}, U_{\delta}\right]=V_{\beta} V_{\beta+2}\left[U_{\beta}, U_{\delta}\right]=V_{\delta-2} V_{\beta+2}\left[U_{\beta}, U_{\delta}\right]
$$

so $\left[W_{\beta+1}, K_{\delta}\right]=1$, and hence $G_{\beta+2} \neq\left\langle G_{\beta+1, \beta+2}, K_{\delta}\right\rangle$. Thus, by $(7)$ and $(3.10)-$ (1), there exists $\xi \in \Delta(\beta+2)$ such that $K_{\delta} \subseteq G_{\xi, \beta+2}, T_{\xi}=T_{\beta+1}$, and $W_{\xi}=$ $W_{\beta+1}$. Hence $\left[U_{\beta}, K_{\delta}\right] \subseteq\left[T_{\xi}, K_{\delta}\right] \subseteq W_{\xi}$ by $(8.5)$, and so $U_{\beta}$ induces a factorwise sesquitransvection on $K_{\delta}$ because $n\left(G_{\delta}, K_{\delta}\right) \geqq 2$ and $\left|W_{\xi}\right|=2^{3}$. Thus $O^{2}\left(G_{\delta} / Q_{\delta} C_{G_{\delta}}\left(K_{\delta}\right)\right) \cong \mathbf{Z}_{3}$ by $(*)$ and $(2.11)-(1)$, which contradicts (7).

$$
T_{\alpha} \cap Q_{\delta-2}=T_{\alpha} \cap Q_{\delta-1} \text { and } D \subseteq W_{\delta-1} .
$$

Proof. Suppose first that $B \cap V_{\delta-2}=1$. Then, since $\left[T_{\alpha} \cap Q_{\delta-2}, U_{\delta-2}\right] \subseteq$ $B \cap V_{\delta-2}=1, T_{\alpha} \cap Q_{\delta-2}=T_{\alpha} \cap Q_{\delta-1}$ by (4) and (7), so $D \subseteq\left[T_{\alpha} \cap Q_{\delta-1}, U_{\delta}\right] \subseteq$ $W_{\delta-1}$. Therefore it is sufficient to prove that $B \cap V_{\delta-2}=1$.

Assume otherwise. Then $B \supset V_{\delta-2}$ by (4), so

$$
W_{\alpha} \supseteqq B=V_{\beta} V_{\delta-2}=W_{\delta-3} \cap W_{\delta-1} \neq V_{\delta-2} \quad \text { and } \quad W_{\delta-1}=B V_{\delta-1}
$$

by (8), (8.7)-(1), and (8.9)-(2). Therefore, by (3.10)-(1), there exists $\lambda \in \Delta(\delta-2)$ such that $T_{\alpha} \subseteq G_{\delta-2, \lambda}, T_{\delta-1}=T_{\lambda}, W_{\delta-1}=W_{\lambda}$, and $V_{\delta-1}=V_{\lambda}$. Thus $\mid T_{\alpha}$ : $T_{\alpha} \cap Q_{\lambda}|\leqq| G_{\delta-2, \lambda}: Q_{\lambda}|\leqq 2=| W_{\alpha} W_{\lambda}: W_{\alpha} \mid$ and $W_{\lambda}=B V_{\lambda}$.

Let $F=\left[T_{\alpha} \cap Q_{\lambda}, U_{\delta} \cap Q_{\beta}\right]$. Since $\left[T_{\alpha} \cap Q_{\lambda}, W_{\lambda}\right]=\left[T_{\alpha} \cap Q_{\lambda}, B V_{\lambda}\right]=1, F \subseteq$ $\left[T_{\alpha} \cap Q_{\lambda}, T_{\lambda} \cap Q_{\beta}\right] \subseteq T_{\alpha} \cap W_{\lambda}$. If $F \varsubsetneqq B$, then $V_{\lambda} \leqq W_{\lambda}=B F \subseteq T_{\alpha}$, so $T_{\alpha} \leqq$ $Q_{\lambda}$. Therefore, $\left|T_{\alpha}: T_{\alpha} \cap Q_{\lambda}\right| \cdot\left|W_{\alpha} F: W_{\alpha}\right| \leqq 2$ both in the case $F \nsubseteq B$ and in the case $F \subseteq B$, so we have $n \leqq 1$, a contradiction.

(10) $W_{\delta-1} \sqsubseteq T_{\alpha}$.

Proof. Since $\left|T_{\alpha}: T_{\alpha} \cap Q_{\delta-1}\right| \cdot\left|W_{\alpha} D: W_{\alpha}\right| \geqq 2^{n} \geqq 2^{2}$ by (2.11)-(2),

(i) $\quad W_{\alpha} \nsupseteq D$ 
by (7) and (9).

Suppose $W_{\delta-1} \nsubseteq T_{\alpha}$. Then

$$
V_{\beta} \subset D=T_{\alpha} \cap W_{\delta-1} \subset W_{\delta-1} \text { and }|D|=2^{2} .
$$

First, we will prove that

(ii) $\quad W_{\delta-1}=D V_{\delta}$ and $V_{\beta} V_{\delta} \nsubseteq V_{\delta-1}$.

Assume otherwise. Then $D \cap V_{\delta-1} \neq V_{\delta-2}$, so $V_{\delta-1}=V_{\delta-2}\left(D \cap V_{\delta-1}\right)$. Thus $\left[T_{\alpha}, V_{\delta-1}\right]=1$. If $U_{\delta-2}=W_{\delta-3} W_{\delta-1}$, then $G_{\delta-2}=\left\langle T_{\alpha}, Q_{\delta-1}\right\rangle$ by $(8.9)-(1)$, and hence $V_{\delta-1} \subseteq Z\left(G_{\delta-2}\right)$, a contradiction. Therefore $U_{\delta-2} \neq W_{\delta-3} W_{\delta-1}=$ $W_{\delta-3} V_{\delta-1}$ by $(8.9)-(2)$, and so $\left[T_{\alpha}, W_{\delta-1}\right]=1$. Thus, by $(3.10)-(1)$, there exists $\xi \in \Delta(\delta-2)$ such that $T_{\alpha} \subseteq G_{\delta-2, \xi}, T_{\xi}=T_{\delta-1}$, and $W_{\xi}=W_{\delta-1}$, so we have $B=\left[T_{\alpha}, U_{\delta-2}\right] \subseteq T_{\alpha} \cap W_{\xi}=D$. Hence $D=V_{\beta} B \subseteq W_{\alpha}$, contrary to (i). This proves (ii).

Now, we will derive a contradiction. Suppose first that $U_{\delta} \nsubseteq Q_{\beta}$. Then, since $V_{\beta} \subset\left[U_{\beta}, U_{\delta}\right] \subseteq T_{\alpha} \cap W_{\delta-1}=D$, we have $\left[U_{\beta}, U_{\delta}\right]=D$. Therefore, by (ii),

(iii) $Z_{\delta-1, \delta} \subseteq U_{\beta} Q_{\delta} \subseteq\left(T_{\alpha} \cap Q_{\delta-1}\right) Q_{\delta} \subseteq E_{\delta-1, \delta}$ and

(iv) $\quad W_{\delta-1} \supset V_{\beta} V_{\delta}=\left[U_{\beta}, U_{\delta} \cap Q_{\beta}\right] V_{\delta} \nsubseteq V_{\delta-1}$.

Thus (iii) together with (2.5)-(8) shows that

$$
U_{\delta} \cap Q_{\beta}=\left[U_{\delta}, G_{\delta-1, \delta}\right] V_{\delta},
$$

but then (iv) is impossible also by (2.5)-(8).

Suppose next that $U_{\delta} \subseteq Q_{\beta}$. Then $U_{\beta}=V_{\alpha}\left(U_{\beta} \cap Q_{\delta}\right)$ by the choice of $\alpha$. Let $H=U_{\delta} \cap Q_{\alpha}$. Then $H=C_{U_{\delta} \bmod V_{\delta}}\left(U_{\beta}\right)$, so

$$
\begin{aligned}
& D V_{\delta}=\left[U_{\beta}, U_{\delta}\right]\left[T_{\alpha} \cap Q_{\delta-1}, H\right] V_{\delta} \quad \text { and } \\
& {\left[U_{\beta}, U_{\delta}\right]\left[T_{\alpha} \cap Q_{\delta-1}, H\right] \subseteq D .}
\end{aligned}
$$

Thus $D=\left[U_{\beta}, U_{\delta}\right]\left[T_{\alpha} \cap Q_{\delta-1}, H\right]=V_{\beta}\left[T_{\alpha} \cap Q_{\delta-1}, H\right] \subseteq W_{\alpha}$, contrary to (i).

(11) A contradiction.

Proof. Take $\theta \in \Delta(\alpha)$ so that $G_{\alpha}=\left\langle G_{\theta \alpha}, U_{\delta} \cap Q_{\beta}\right\rangle$. Let $x \in O^{2}\left(G_{\theta}\right)$ so that $C_{U_{\theta} / V_{\theta}}(x)=1$, let $t \in Z_{\alpha^{x}, \theta}-Q_{\theta}$, and put $\kappa=\alpha^{t}$. Then $W_{\kappa} \cap W_{\alpha}=V_{\theta}$ by (2.5)-(11), and $t$ permutes $T_{\kappa}$ and $T_{\alpha}$ because $t$ inverts $\langle x\rangle Q_{\theta} / Q_{\theta}$. Since $\left[W_{\kappa}, U_{\delta-4}\right]$ $\subseteq\left[U_{\theta}, U_{\delta-4}\right]=1$, we have $\left[T_{\kappa}, U_{\delta-4}\right] \subseteq C_{W_{\kappa}}\left(U_{\delta}\right) \subseteq W_{\alpha}$ by $(8.5)$, so $\left[T_{\kappa}, U_{\delta-4}\right]$ $\subseteq C_{W_{\kappa} \cap W_{\alpha}}\left(U_{\delta}\right)=C_{V_{\theta}}\left(U_{\delta}\right)=1$. Thus $T_{\kappa} \subseteq C_{Q_{\delta-5}}\left(U_{\delta-4}\right) \subseteq Q_{\delta-3}$.

Now, let $K=T_{\kappa} T_{\alpha}$. Note that $K$ is abelian as $b \geqq 7$. Since $\left[K, W_{\delta-1}\right]=1$ by (10), we have $G_{\delta-3} \neq\left\langle K, G_{\delta-2, \delta-1}\right\rangle$, so there exists $\xi \in \Delta(\delta-2)$ such that $K \subseteq G_{\delta-2, \xi}, T_{\xi}=T_{\delta-1}$, and $W_{\xi}=W_{\delta-1}$, and hence $\left[K, T_{\xi}\right] \subseteq W_{\xi} \subseteq K$. Therefore $K \triangleleft\left\langle Q_{\theta}, U_{\delta} \cap Q_{\beta}\right\rangle=G_{\alpha}$, and so $K \triangleleft G_{\alpha}{ }^{t}=G_{\kappa}$. This shows that $K \triangleleft\left\langle G_{\kappa}, G_{\alpha}\right\rangle=\left\langle G_{\theta}, G_{\alpha}\right\rangle$, a contradiction. This completes the proof of (8.10). 
Now, let $\mathcal{C}_{0}{ }^{\prime}=\left\{(\beta, \delta) \in \mathcal{C}^{\prime} \mid U_{\delta} \nsubseteq Q_{\beta}\right\}$.

Corollary 8.11 Let $\beta, \delta \in \mathcal{B}$ and $d(\beta, \delta) \leqq b-1$. Then the following holds.

(1) Either $(\beta, \delta) \in \mathcal{C}_{0}{ }^{\prime}$ or $\left[U_{\beta}, U_{\delta}\right]=1$

(2) $U_{\delta} \cap Q_{\beta}=C_{U_{\delta}}\left(U_{\beta}\right)=C_{U_{\delta} \bmod V_{\delta}}\left(U_{\beta}\right)$.

(3) $\left|U_{\beta}: U_{\beta} \cap Q_{\delta}\right|=\left|U_{\delta}: U_{\delta} \cap Q_{\beta}\right|$.

(4) $Z_{\delta-1, \delta} \neq U_{\beta} Q_{\delta} \subseteq E_{\delta-1, \delta}$.

(5) If $U_{\beta} \subseteq M \cong E_{\delta-1, \delta}$, then $\left[M, U_{\delta}\right] \subseteq\left[U_{\beta}, U_{\delta}\right]\left[M, U_{\delta} \cap Q_{\beta}\right] V_{\delta}$.

(6) If $(\beta, \delta) \in \mathcal{C}_{0}{ }^{\prime}$, then $K_{\beta} \cap G_{\delta-1, \delta} \leqq E_{\delta-1, \delta}$.

Proof. Suppose $\left[U_{\beta}, U_{\delta}\right] \neq 1$. Then we have $U_{\beta} \nsubseteq Q_{\delta}$ and $U_{\delta} \nsubseteq Q_{\beta}$ by (8.10), and then $d(\beta, \delta) \geqq b-1$ by the minimality of $b$. This shows that $(\beta, \delta) \in \mathcal{C}_{0}{ }^{\prime}$, so (1) holds.

In proving $(2)-(6)$, we may assume that $\left[U_{\beta}, U_{\delta}\right] \neq 1$, and, by symmetry, that $\left|U_{\beta}: U_{\beta} \cap Q_{\delta}\right| \geqq\left|U_{\delta}: U_{\delta} \cap Q_{\beta}\right|$. Let $\widetilde{G}_{\delta}=G_{\delta} / C_{G_{\delta}}\left(U_{\delta} / V_{\delta}\right)$ and $\widehat{U}_{\delta}=U_{\delta} / V_{\delta}$. Then we have

$$
\left|\widehat{U}_{\delta}: C_{\widehat{U}_{\delta}}\left(\widetilde{U}_{\beta}\right)\right| \leqq\left|U_{\delta}: U_{\delta} \cap Q_{\beta}\right| \leqq\left|U_{\beta}: U_{\beta} \cap Q_{\delta}\right|=\left|\widetilde{U}_{\beta}\right|
$$

by $(6.3),(2.4)-(1)$, and (8.10). Hence $\left|\widetilde{U}_{\beta}\right|=\left|\widehat{U}_{\delta}: C_{\widehat{U}_{\delta}}\left(\widetilde{U}_{\beta}\right)\right|$ by $(2.10)-(1)$, and so (2) and (3) holds. Since $\left(G_{\delta},\left[\widehat{U}_{\delta}, O^{2}\left(G_{\delta}\right)\right]\right) \ll\left(O_{4}^{+}(2), G F(2)^{4}\right)$ by $(8.3)-(2),(4)$ and (5) follow from (5) and (6) of (2.5), respectively. Let $K=K_{\beta} \cap G_{\delta-1, \delta}$. Since $K_{\beta}$ centralizes $U_{\beta}$ by the minimality of $b, \widetilde{K} \subseteq C_{\widetilde{G}_{\delta-1, \delta}}\left(\widetilde{U}_{\beta}\right) \subseteq \widetilde{E}_{\delta-1, \delta}$ by (4), so (6) holds.

Lemma $8.12 \operatorname{Let}(\beta, \delta) \in \mathcal{C}_{0}{ }^{\prime}$, and suppose there exist $\alpha \in \Delta(\beta)$ and $M \triangleleft G_{\alpha \beta}$ such that

$$
M \not G_{\beta}=\left\langle G_{\alpha \beta}, U_{\delta}\right\rangle \text { and } U_{\beta} \subseteq M \subseteq T_{\alpha} \cap Q_{\delta-1} .
$$

Define $K=\left\langle M^{G_{\beta}}\right\rangle$. Then the following holds.

(1) $\quad M \nsupseteq V_{\delta} \subseteq\left[M, U_{\delta}\right] \subseteq M V_{\delta} \subseteq K$.

(2) If $U_{\beta} \subseteq K^{*} \subseteq K$ and $V_{\delta} \subseteq K^{*} \triangleleft G_{\beta}$, then $\left[K, O^{2}\left(G_{\beta}\right)\right] \subseteq K^{*}$.

Proof. Let $N=\left[U_{\beta}, U_{\delta}\right]\left[M, U_{\delta} \cap Q_{\beta}\right]$. Then $M \supseteqq N \cong\left[M, U_{\delta}\right] \subseteq N V_{\delta}$ by (8.11)-(5)(6), and $\left|N V_{\delta}: N\right| \leqq 2$. Therefore, since $U_{\delta}$ does not normalize $M$, $\left[M, U_{\delta}\right]=N V_{\delta}$ and $V_{\delta} \varsubsetneqq M$, so $V_{\delta} \subseteq\left[M, U_{\delta}\right] \subseteq M V_{\delta} \subseteq K$. Thus (1) holds.

Let $K^{*} \subseteq K^{\prime} \subseteq K$ and assume $K^{\prime} \triangleleft G_{\alpha \beta}$. Since $\left[M, U_{\delta}\right] \subseteq M V_{\delta} \subseteq M K^{*}$, $K=M K^{*}$, so $K^{\prime}=\left(M \cap K^{\prime}\right) K^{*}$. Thus we have $\left[K^{\prime}, U_{\delta}\right] \subseteq\left[M \cap K^{\prime}, U_{\delta}\right] K^{*} \subseteq$ $\left(M \cap K^{\prime}\right) V_{\delta} K^{*}=K^{\prime}$. Therefore all $G_{\alpha \beta^{-}}$invariant subgroups between $K^{*}$ and $K$ are normal in $G_{\beta}$, and so $\left[K, O^{2}\left(G_{\beta}\right)\right] \subseteq K^{*}$. Hence (2) holds.

Proposition 8.13 Let $\xi \in \mathcal{A}$ and $\eta \in \mathcal{B}$. Then

$$
\left|W_{\xi}\right|=2^{3} \text { and }\left|U_{\eta}\right|=2^{5} .
$$


Proof. We may assume $\langle\xi, \eta\rangle \in E(\Gamma)$. Define $K_{\eta}{ }^{*}=\left\langle T_{\xi}{ }^{(2)^{G} \eta}\right\rangle$.

$$
\begin{array}{r}
\text { Let } \beta, \mu \in \mathcal{B} \text { and } d(\beta, \mu) \leqq b-3 \text {. Then } \\
{\left[K_{\beta}{ }^{*}, U_{\mu}\right]=1 .}
\end{array}
$$

Proof. Let $\alpha \in \Delta(\beta)$ and $\theta \in \Delta(\alpha)$. Then $d(\theta, \mu) \leqq b-1$, so we have $\left[U_{\theta}, Q_{\alpha} ; 2\right] \subseteq C_{U_{\theta} \bmod V_{\theta}}\left(U_{\mu}\right)=C_{U_{\theta}}\left(U_{\mu}\right)$ by $(8.11)-(1)(4)$ and $(2.5)-(4)$. Since $K_{\beta}{ }^{*}=\left\langle\left[U_{\theta}, Q_{\alpha} ; 2\right] \mid \alpha \in \Delta(\beta), \theta \in \Delta(\alpha)\right\rangle$, we have $\left[K_{\beta}{ }^{*}, U_{\mu}\right]=1$.

(2) One of the following holds.

(i) There exist $(\beta, \delta) \in \mathcal{C}_{0}{ }^{\prime}$ and $\alpha \in \Delta(\beta)$ such that $G_{\beta}=\left\langle G_{\alpha \beta}, U_{\delta}\right\rangle$ and $\left[T_{\alpha}, U_{\delta-2}\right]=1$.

(ii) There exist $(\beta, \delta) \in \mathcal{C}_{0}{ }^{\prime}, \alpha \in \Delta(\beta)$ and $\theta \in \Delta(\alpha)$ such that $G_{\beta}=$ $\left\langle G_{\alpha \beta}, U_{\delta}\right\rangle, G_{\alpha}=\left\langle G_{\theta \alpha}, G_{\alpha \beta}\right\rangle$, and $U_{\theta} \nsubseteq Q_{\delta-2}$.

(iii) $\left|W_{\xi}\right|=2^{3}$ for $\xi \in \mathcal{A}$.

Proof. Suppose that neither (i) nor (ii) holds.

Let $(\beta, \delta) \in \mathcal{C}_{0}{ }^{\prime}$, and take $\alpha \in \Delta(\beta)$ so that $G_{\beta}=\left\langle G_{\alpha \beta}, U_{\delta}\right\rangle$. Then $T_{\alpha} \nsubseteq$ $Q_{\delta-2}$ by our hypothesis. Take $\theta \in \Delta(\alpha)$ so that $U_{\theta} \nsubseteq Q_{\delta-2}$. Then $(\theta, \delta-2) \in \mathcal{C}_{0}{ }^{\prime}$, and $G_{\alpha} \neq\left\langle G_{\theta \alpha}, G_{\alpha \beta}\right\rangle$ also by our hypothesis, and so $V_{\theta}=V_{\beta}$ by (3.10)-(2). Thus, changing notation, there exists $(\beta, \delta) \in \mathcal{C}_{0}{ }^{\prime}$ such that $V_{\beta}=V_{\beta+2}=\cdots=V_{\delta-2}=V_{\delta}$.

Now, let $(\beta, \delta) \in \mathcal{C}_{0}{ }^{\prime}$ and $\alpha \in \Delta(\beta)$ so that $G_{\beta}=\left\langle G_{\alpha \beta}, U_{\delta}\right\rangle$ and $U_{\beta} \supseteqq V_{\delta}$. Then $T_{\alpha} \subseteq K_{\beta}{ }^{*} \subseteq C_{Q_{\delta-3}}\left(U_{\delta-2}\right) \subseteq Q_{\delta-1}$ by (1), and so $T_{\alpha}^{(2)} U_{\beta} \triangleleft G_{\beta}$ by (8.12)(1). Since $\left[T_{\alpha}{ }^{(2)}, Q_{\alpha}, Q_{\alpha}\right] \subseteq W_{\alpha}$ by (8.4) and $n_{*}\left(G_{\beta}\right)>1, G_{\beta}$ has no noncentral chief factor within $T_{\alpha}{ }^{(2)} U_{\beta} / U_{\beta}$ by (2.12)-(1), and so $\left[T_{\alpha}^{(2)} U_{\beta}, O^{2}\left(G_{\beta}\right)\right] \subseteq U_{\beta}$. Thus $\left[T_{\alpha}{ }^{(2)}, O^{2}\left(G_{\alpha}\right)\right] \subseteq W_{\alpha}$ by (3.8). Therefore $T_{\alpha}{ }^{(2)}=W_{\alpha}$ by $(2.14)-(1)$, so $\left|W_{\alpha}\right|=2^{3}$ by (8.4). Hence (iii) holds.

(3) Part (iii) of (2) holds.

Proof. Suppose false. Then either (i) or (ii) of (2) holds.

First, let $(\beta, \delta) \in \mathcal{C}_{0}{ }^{\prime}$ and $\alpha \in \Delta(\beta)$ as in (i) of (2). Since $\left[T_{\alpha}{ }^{(2)}, Q_{\alpha}, Q_{\alpha}\right] \subseteq$ $W_{\alpha} \nsupseteq\left[T_{\alpha}{ }^{(2)}, O^{2}\left(G_{\alpha}\right)\right], T_{\alpha}{ }^{(2)} U_{\beta}, \Varangle G_{\beta}$ by (3.8). Thus $V_{\delta} \subseteq K_{\beta}{ }^{*}$ by (8.12)-(1), and hence $\left[K_{\beta}, O^{2}\left(G_{\beta}\right)\right] \subseteq K_{\beta}{ }^{*}$ by $(8.12)-(2)$. Therefore $\left[U_{\beta}, U_{\delta}\right] \subseteq\left[K_{\beta+2}, U_{\delta}\right]=$ $\left[K_{\beta+2}{ }^{*} T_{\beta+3}, U_{\delta}\right]=1$ by $(1)$ and the minimality of $b$, a contradiction.

Next, let $(\beta, \delta) \in \mathcal{C}_{0}{ }^{\prime}, \alpha \in \Delta(\beta)$, and $\theta \in \Delta(\alpha)$ as in (ii) of (2). Then $G_{\alpha \beta}=$ $\left(Q_{\theta} \cap Q_{\alpha}\right) Q_{\beta}$ by (3.2)-(3), and so $\left\langle Q_{\theta} \cap Q_{\alpha}, U_{\delta}\right\rangle$ contains a Sylow 3-subgroup, say $P$, of $G_{\beta}$. Define $F=\left[U_{\theta}, U_{\delta-2}\right]$ and $L=\cap\left(T_{\alpha}{ }^{(2)} U_{\beta}\right)^{G_{\beta}}$. Then, as $F \subseteq\left[U_{\theta}, Q_{\alpha} ; 2\right]$ $\subseteq T_{\alpha}{ }^{(2)}$ and $U_{\theta} \supseteqq V_{\alpha} F \supseteqq V_{\theta} \subseteq U_{\beta},\left[U_{\beta} F, Q_{\theta} \cap Q_{\alpha}\right] \subseteq U_{\beta} \supseteq\left[U_{\beta} F, U_{\delta}\right]$, so $U_{\beta} F \subseteq \cap\left(T_{\alpha}{ }^{(2)} U_{\beta}\right)^{P}=\cap\left(T_{\alpha}{ }^{(2)} U_{\beta}\right)^{G_{\beta}}=L$. Moreover, since $\left[L, Q_{\beta}, Q_{\beta}\right] \subseteq U_{\beta}$ and $n_{*}\left(G_{\beta}\right)>1$, we have also $\left[L, O^{2}\left(G_{\beta}\right)\right] \subseteq U_{\beta}$. Let $H=\left[Q_{\beta}, O^{2}\left(G_{\beta}\right)\right]$. Then 
$[F, H] \subseteq\left[T_{\alpha}{ }^{(2)} \cap L, H\right] \subseteq W_{\alpha}$ by (3.8), and so $\left[F,\left\langle Q_{\theta}, H\right\rangle\right] \subseteq W_{\alpha}$. Therefore, since $\left\langle Q_{\theta}, H\right\rangle$ contains a Sylow 3 -subgroup, say $R$, of $G_{\alpha}, V_{\alpha} \neq V_{\alpha} F \subseteq W_{\alpha} F \subseteq$ $\cap U_{\theta}{ }^{R} \subseteq \cap U_{\theta}{ }^{G_{\alpha}}=W_{\alpha}$, a contradiction.

(8.14) Let $(\beta, \delta) \in \mathcal{C}_{0}{ }^{\prime}$ and suppose $K_{\beta} \subseteq Q_{\delta-1}$. Then $\left|T_{\delta-1}: T_{\delta-1} \cap Q_{\beta}\right|=2$, and $\left(G_{\eta}, K_{\eta} / U_{\eta}\right) \ll\left(O_{4}^{+}(2), G F(2)^{4}\right)$ and $n\left(G_{\eta}, K_{\eta} / U_{\eta}\right)=1$ for $\eta \in \mathcal{B}$.

Proof. We may assume $\eta=\beta$. Since $W_{\delta-1}=V_{\delta-1}\left[U_{\beta}, U_{\delta}\right]$ by $(2.5)-(7)$ and (8.11)-(4), $K_{\beta}$ centralizes $W_{\delta-1}$, and so

(i) $\left[K_{\beta}, T_{\delta-1}\right] \subseteq W_{\delta-1}$

by (8.5). Since $K_{\beta}$ centralizes $U_{\beta}$ by the minimality of $b$,

(ii) $\left[K_{\beta}, U_{\delta}\right] \subseteq\left[U_{\beta}, U_{\delta}\right]\left[K_{\beta}, U_{\delta} \cap Q_{\beta}\right] V_{\delta} \subseteq U_{\beta} V_{\delta}$

by $(2.5)-(6)$ and $(8.11)-(2)$. Hence (ii) shows that $n\left(G_{\beta}, K_{\beta} / U_{\beta}\right)=1$, and so $n\left(G_{\beta}, K_{\beta}\right)=2$. Thus $T_{\delta-1}$ induces a factorwise sesquitransvection on $K_{\beta}$ by (i) as $\left|W_{\delta-1}\right|=2^{3}$, and hence $\left(G_{\beta}, K_{\beta}\right) \ll\left(O_{4}^{+}(2), G F(2)^{4}\right)$ and $\left|T_{\delta-1}: T_{\delta-1} \cap Q_{\beta}\right|=2$.

(8.15) Let $(\beta, \delta) \in \mathcal{C}_{0}{ }^{\prime}$ and $\alpha \in \Delta(\beta)$ so that $W_{\alpha} \cap W_{\beta+1}=V_{\beta}$. If $\mid K_{\delta-2}$ : $K_{\delta-2} \cap Q_{\beta} \mid=2^{2}$, then $\left[T_{\alpha}, U_{\delta-2}\right]=1$.

Proof. Suppose $\left|K_{\delta-2}: K_{\delta-2} \cap Q_{\beta}\right|=2^{2}$. Then, as $C_{U_{\beta}}\left(K_{\delta-2}\right)=W_{\beta+1}$, $\left[T_{\alpha}, U_{\delta-2}\right] \subseteq C_{W_{\alpha}}\left(K_{\delta-2}\right)=W_{\alpha} \cap W_{\beta+1}=V_{\beta} \subseteq V_{\alpha}$. Thus $U_{\delta-2} \subseteq Q_{\theta}$ for all $\theta \in \Delta(\alpha)$ by $(2.5)-(9)$, and hence $\left[T_{\alpha}, U_{\delta-2}\right]=1$ by $(8.11)-(1)$.

(8.16) Let $(\beta, \delta) \in \mathcal{C}_{0}{ }^{\prime}$. Then

$$
\left|T_{\beta+1}: T_{\beta+1} \cap Q_{\delta}\right|=\left|T_{\delta-1}: T_{\delta-1} \cap Q_{\beta}\right|=2 .
$$

Proof. Suppose false. Take $(\beta, \delta) \in \mathcal{C}_{0}{ }^{\prime}$ and $\alpha \in \Delta(\beta)$ so that $\mid T_{\delta-1}: T_{\delta-1} \cap$ $Q_{\beta} \mid=2^{2}$ and $W_{\alpha} \cap W_{\beta+1}=V_{\beta}$. Define

$$
M=T_{\alpha}^{(1)} U_{\beta}, L=\left\langle M^{T_{\delta-1}}, \quad \text { and } \quad K=\left\langle M^{G_{\beta}}\right\rangle .\right.
$$

(1) $|L: M| \leqq 2$.

Proof. Since $\left[M, Q_{\alpha}\right] \subseteq U_{\beta}$ and $\left[T_{\alpha}^{(1)}, O^{2}\left(G_{\alpha}\right)\right] \varsubsetneqq W_{\alpha}$, we have $M \not G_{\beta}$ by (3.8). Moreover, $G_{\beta}=\left\langle G_{\alpha \beta}, U_{\delta}\right\rangle$ by the choice of $\alpha$ and (8.9)-(1), and $M \subseteq$ $T_{\alpha} \subseteq C_{Q_{\delta-3}}\left(U_{\delta-2}\right) \subseteq Q_{\delta-1}$ by (8.5) and (8.15). Thus (8.12)-(1) shows that $V_{\delta} \nsubseteq M$, so $V_{\delta} \varsubsetneqq W_{\beta+1}$. Since $W_{\beta+1}=V_{\beta}\left[U_{\beta}, T_{\delta-1}\right]$ and $\left[U_{\beta}, T_{\delta-1}\right] \leqq W_{\beta+1} \cap$ $W_{\delta-1}$, we have $\left|W_{\delta-1}: W_{\beta+1} \cap W_{\delta-1}\right| \leqq 2$, and hence $W_{\delta-1}=\left(W_{\beta+1} \cap W_{\delta-1}\right) V_{\delta}$. Therefore $\left\langle M^{T_{\delta-1}}\right\rangle=M\left[M, T_{\delta-1}\right] \subseteq M W_{\delta-1}=M V_{\delta}$, and so $|L: M| \leqq 2$.

(2) A contradiction. 
Proof. Let $K / N$ be a noncentral chief factor of $G_{\beta}$ within $K / U_{\beta}$. Since $1 \neq M N / N \subseteq C_{K / N}\left(G_{\alpha \beta}\right) \neq K / N$ and $G_{\beta}=\left\langle G_{\alpha \beta}, T_{\delta-1}\right\rangle$, we have $L N \neq M N$, so $|L N: M N|=2$ and $|L N / N| \geqq 2^{2}$ by (1). Let $F / N$ be a $T_{\delta-1}$-invariant fours subgroup of $L / N$, and set $C=C_{T_{\delta-1}}(F / N)$. Since $\left|T_{\delta-1}: T_{\delta-1} \cap Q_{\beta}\right|=2^{2}$, we have $C \nsubseteq Q_{\beta}$, and hence $G_{\beta}=\left\langle G_{\alpha \beta}, C\right\rangle$ by the choice of $\alpha$ and (8.9)-(1). Thus we have $1 \neq M N / N \cap F / N \subseteq C_{K_{\beta} / N}\left(G_{\beta}\right)$, a contradiction.

(8.17) We have $b \geqq 7$ (and hence $K_{\eta}$ is elementary abelian for $\eta \in \mathcal{B}$ ).

Proof. Suppose $b=5$, and let $(\beta, \delta) \in \mathcal{C}_{0}{ }^{\prime}$. Since $Q_{\delta-3} \triangleright K_{\delta-2} \nsubseteq Q_{\beta}$, we have $K_{\delta-2} Q_{\beta}=E_{\beta, \delta-3}$. Take $\alpha \in \Delta(\beta)$ so that $W_{\alpha} \cap W_{\beta+1}=V_{\beta}$. Then $\left[T_{\alpha}, U_{\delta-2}\right]=1$ by (8.15), and hence $V_{\delta} \nsubseteq U_{\beta}$ by (8.12)-(1). Thus $V_{\delta-2} \neq V_{\delta}$, and so $G_{\delta-1}=$ $\left\langle G_{\delta-2, \delta-1}, G_{\delta-1, \delta}\right\rangle$ by $(8.3)-(1)$ and $(3.10)-(2)$, so $G_{\delta-1, \delta}=\left(Q_{\delta-2} \cap Q_{\delta-1}\right) Q_{\delta}$ by (3.2)-(3). Therefore $T_{\delta-3} Q_{\delta} \triangleleft G_{\delta-1, \delta}$, and hence $\left|T_{\delta-1}: T_{\delta-1} \cap Q_{\beta}\right|=2^{2}$, contrary to $(8.16)$.

Proposition 8.18 Let $\eta \in \mathcal{B}$. Then $n\left(G_{\eta}, K_{\eta} / U_{\eta}\right) \leqq 2$ and $\left(G_{\eta}, K_{\eta} / U_{\eta}\right) \ll$ $\left(O_{4}^{+}(2), G F(2)^{4}\right)$.

Proof. Suppose false.

$$
K_{\beta} \nsubseteq Q_{\delta-2} \text { for all }(\beta, \delta) \in \mathcal{C}_{0}{ }^{\prime} .
$$

Proof. Suppose $K_{\beta} \subseteq Q_{\delta-2}$ for some $(\beta, \delta) \in \mathcal{C}_{0}{ }^{\prime}$. Then $K_{\beta} \subseteq C_{Q_{\delta-2}}\left(U_{\delta-2}\right)$ $\subseteq Q_{\delta-1}$ by (8.11)-(1) and (8.5). Hence (8.18) holds by (8.14).

(2) $\left|K_{\beta}: H\right| \cdot\left|\left[H, U_{\delta}\right] U_{\beta}: U_{\beta}\right| \geqq 2^{3}$ for all $(\beta, \delta) \in \mathcal{C}_{0}{ }^{\prime}$ and all $U_{\delta}$-invariant subgroups $H$ between $U_{\beta}$ and $K_{\beta}$.

Proof. Suppose false. Then $n\left(G_{\beta}, K_{\beta} / U_{\beta}\right) \leqq 2$ by $(2.11)-(2)$, and $U_{\delta}$ induces a factorwise quasitransvection on $K_{\beta} / U_{\beta}$. Thus (8.18) holds by (2.11)-(1) because $G_{\beta}$ is a $\{2,3\}$-group and $U_{\delta} Q_{\beta} \subseteq Z_{\beta, \beta+1}$ by (8.11)-(4).

(3) There exist $(\beta, \delta) \in \mathcal{C}_{0}{ }^{\prime}$ and $\alpha \in \Delta(\beta)$ such that

$$
T_{\alpha} \nsubseteq Q_{\delta-2} \quad \text { and } \quad G_{\beta}=\left\langle G_{\alpha \beta}, G_{\beta, \beta+1}\right\rangle \text {. }
$$

Proof. Suppose false. Let $(\beta, \delta) \in \mathcal{C}_{0}{ }^{\prime}$. We can take, by (1), $\alpha \in \Delta(\beta)$ so that $T_{\alpha} \nsubseteq Q_{\delta-2}$. Then we have $G_{\beta} \neq\left\langle G_{\alpha \beta}, G_{\beta, \beta+1}\right\rangle$ by our present hypothesis, and so $V_{\alpha}=V_{\beta+1}$ by (8.3)-(2) and (3.10)-(2). Take $\theta \in \Delta(\alpha)$ so that $U_{\theta} \nsubseteq Q_{\delta-2}$. Then $(\theta, \delta-2) \in \mathcal{C}_{0}{ }^{\prime}$. Therefore, changing notation, there exists $(\beta, \delta) \in \mathcal{C}_{0}{ }^{\prime}$ such that

$$
U_{\beta} \supseteqq V_{\beta+1}=V_{\beta+3}=\cdots=V_{\delta-3}=V_{\delta-1} \supseteq V_{\delta} .
$$

Now, let $(\beta, \delta) \in \mathcal{C}_{0}{ }^{\prime}$ and $\alpha \in \Delta(\beta)$ so that $U_{\beta} \supseteq V_{\delta}$ and $G_{\beta}=\left\langle G_{\alpha \beta}, U_{\delta}\right\rangle$. 
Then $T_{\alpha} \subseteq Q_{\delta-2}$ by our hypothesis, and hence $T_{\alpha} \subseteq C_{Q_{\delta-2}}\left(U_{\delta-2}\right) \subseteq Q_{\delta-1}$ by the minimality of $b$ and (8.11)-(1). Thus $T_{\alpha} \triangleleft G_{\beta}$ by (8.12)-(1), a contradiction.

Now, take $\alpha \in \Delta(\beta)$ and $\theta \in \Delta(\alpha)$ so that

$$
G_{\beta}=\left\langle G_{\alpha \beta}, G_{\beta, \beta+1}\right\rangle \text { and } \quad U_{\theta} \varsubsetneqq Q_{\delta-2} .
$$

(4) $\left|K_{\delta-2}: K_{\delta-2} \cap Q_{\beta}\right|=2^{2}$.

Proof. Suppose $\left|K_{\delta-2}: K_{\delta-2} \cap Q_{\beta}\right|=2$. Since $V_{\beta} \nsupseteq\left[T_{\alpha}, U_{\delta-2}\right] \subseteq W_{\alpha} \cap$ $U_{\delta-2}$, and since $V_{\beta} \subseteq U_{\delta-2}$ if $K_{\delta-2} \cap Q_{\beta} \nsubseteq Q_{\alpha}$, we have

$$
\left|K_{\delta-2}: K_{\delta-2} \cap Q_{\alpha}\right| \cdot\left|W_{\alpha}: W_{\alpha} \cap U_{\delta-2}\right| \leqq 2^{3} .
$$

Moreover, we have $\left[U_{\theta}, K_{\delta-2} \cap Q_{\alpha}\right] \subseteq W_{\alpha}$ because $K_{\delta-2} \cap Q_{\alpha} \subseteq E_{\theta \alpha}$ by (8.11)-(6), so

$$
V_{\alpha} \subseteq W_{\alpha}=\left[U_{\theta}, K_{\delta-2} \cap Q_{\alpha}\right]\left(W_{\alpha} \cap U_{\delta-2}\right) \subseteq K_{\delta-2},
$$

by (2) and the above inequality. Thus $K_{\delta-2} \cap Q_{\beta} \subseteq Q_{\alpha}$, and hence $\left[U_{\theta}, K_{\delta-2} \cap Q_{\beta}\right]$ $\subseteq\left[U_{\theta}, U_{\delta-2}\right]\left[U_{\theta} \cap Q_{\delta-2}, K_{\delta-2} \cap Q_{\beta}\right] V_{\theta} \subseteq U_{\delta-2} V_{\theta}$.

Consequently, we have

$$
\left|K_{\delta-2}: K_{\delta-2} \cap Q_{\beta}\right| \cdot\left|\left[U_{\theta}, K_{\delta-2} \cap Q_{\beta}\right] U_{\delta-2}: U_{\delta-2}\right| \leqq 2 \cdot 2=2^{2},
$$

contrary to (2).

Let $L_{\eta}=\cap T_{\xi}^{G_{\eta}}$ for $\langle\xi, \eta\rangle \in E(\Gamma)$.

(5) $\left|T_{\xi}^{(1)} L_{\eta}: L_{\eta}\right| \leqq 2$.

Proof. We may assume $\eta=\beta$, and $W_{\xi} \cap W_{\beta+1}=V_{\beta}$. Let $x \in U_{\delta}-Q_{\beta}$ and put $M=T_{\xi}^{(1)} L_{\beta}$. By (4) and (8.15), $\left[M, U_{\delta-2}\right] \subseteq\left[T_{\xi}, U_{\delta-2}\right]=1$, and so $M\left[M, U_{\delta}\right]$ $\subseteq M V_{\delta}$ by (8.12)-(1). Therefore we have $\left|M: M \cap M^{x}\right| \leqq 2$. Since $\left[T_{\xi}^{(1)}, Q_{\xi}\right]$ $\subseteq W_{\xi} \subseteq U_{\beta}$ and $G_{\beta}=\left\langle G_{\xi \beta}, x\right\rangle=\left\langle Q_{\xi}, Q_{\xi}{ }^{x}\right\rangle$ by (8.9)-(1) and (3.2)-(3), we have $\left[M \cap M^{x}, G_{\beta}\right] \subseteq U_{\beta}$, and so $M \cap M^{x} \subseteq L_{\beta}$. Hence we have $\left|T_{\xi}^{(1)} L_{\beta}: L_{\beta}\right| \leqq 2$.

(6) $\quad O^{2}\left(G_{\xi} / C_{G_{\xi}}\left(T_{\xi}^{(1)} / W_{\xi}\right)\right) \cong \mathbf{Z}_{3}$ for $\xi \in \mathcal{A}$.

Proof. This follows from (2.11)-(1) because $G_{\xi}$ is a $\{2,3\}$-group with cyclic Sylow 3 -subgroups and $\left[Q_{\eta}, O^{2}\left(G_{\eta}\right)\right]$ induces a transvection on $T_{\xi}^{(1)} / W_{\xi}$ by $(5)$ and (3.8), where $\eta \in \Delta(\xi)$.

(7) $\left|K_{\beta}: K_{\beta} \cap Q_{\delta-2}\right|=2$.

Proof. Suppose $U_{\theta} \cap T_{\alpha}{ }^{(1)}=U_{\beta} \cap T_{\alpha}{ }^{(1)}$. Then we have $\left[\left[U_{\theta}, G_{\theta \alpha}\right], U_{\delta-2}\right]=$ $\left[U_{\theta} \cap T_{\alpha}^{(1)}, U_{\delta-2}\right] \subseteq\left[U_{\beta}, U_{\delta-2}\right]=1$ by the minimality of $b$. Hence $U_{\delta-2} \subseteq Q_{\theta}$ by 
(2.5)-(9), and so $U_{\theta} \subseteq C_{Q_{\delta-3}}\left(U_{\delta-2}\right) \subseteq Q_{\delta-2}$, a contradiction. Thus $U_{\theta} \cap T_{\alpha}{ }^{(1)} \neq$ $U_{\beta} \cap T_{\alpha}{ }^{(1)}$, and so $G_{\alpha}=\left\langle G_{\theta \alpha}, G_{\alpha \beta}\right\rangle$ by (6) and (3.10)-(2), and then $V_{\alpha}=V_{\theta} V_{\beta}$.

Now, suppose $\left|K_{\beta}: K_{\beta} \cap Q_{\delta-2}\right|=2^{2}$. Let $\lambda \in \Delta(\delta-2)$ so that $W_{\delta-3} \cap$ $W_{\lambda}=V_{\delta-2}$. Then, by (8.15), $\left[U_{\beta}, T_{\lambda}\right]=1$, and so $T_{\lambda} \subseteq Q_{\alpha}$. Since $T_{\lambda} \not G_{\delta-2}=$ $\left\langle U_{\theta}, G_{\delta-2, \lambda}\right\rangle$, we have $V_{\theta} \subseteq K_{\delta-2}$ by $(8.12)-(1)$, and so $\left[V_{\alpha}, K_{\delta-2}\right]=1$. As $G_{\beta}=$ $\left\langle G_{\alpha \beta}, G_{\beta, \beta+1}\right\rangle=\left\langle Q_{\alpha}, K_{\delta-2}\right\rangle$ by (4) and the choice of $\alpha, V_{\alpha} \subseteq Z\left(G_{\beta}\right)$, a contradiction.

(8) A contradiction.

Proof. If $G_{\delta-2}=\left\langle G_{\delta-3, \delta-2}, G_{\delta-2, \delta-1}\right\rangle$, then, for $(\delta-2, \theta) \in \mathcal{C}_{0}{ }^{\prime}$ and $\delta-1 \epsilon$ $\Delta(\delta-2)$, we have $\left|K_{\beta}: K_{\beta} \cap Q_{\delta-2}\right|=2^{2}$ by (4), contrary to (7). Thus $G_{\delta-2} \neq$ $\left\langle G_{\delta-3, \delta-2}, G_{\delta-2, \delta-1}\right\rangle$, and hence $W_{\delta-3}=W_{\delta-1}$ by $(3.10)-(1)$. Therefore $\left[K_{\beta} \cap\right.$ $\left.Q_{\delta-2}, W_{\delta-1}\right]=1$, and so

$$
\left[K_{\beta} \cap Q_{\delta-2}, U_{\delta}\right] \subseteq\left[U_{\beta}, U_{\delta}\right]\left[K_{\beta} \cap Q_{\delta-2}, U_{\delta} \cap Q_{\beta}\right] V_{\delta} \subseteq U_{\beta} V_{\delta}
$$

Thus $\left|K_{\beta}: K_{\beta} \cap Q_{\delta-2}\right| \cdot\left|\left[K_{\beta} \cap Q_{\delta-2}, U_{\delta}\right] U_{\beta}: U_{\beta}\right| \leqq 2 \cdot 2=2^{2}$, contrary to (2).

Corollary 8.19 Let $\xi \in \mathcal{A}$. Then $O^{2}\left(G_{\xi} / C_{G_{\xi}}\left(T_{\xi}^{(1)} / W_{\xi}\right)\right) \cong \mathbf{Z}_{3}$. have

Proof. Define $L_{\eta}=\cap T_{\xi} G_{\eta}$ for $\eta \in \triangle(\xi)$. Then, by (8.18) and (2.15), we

$$
\left|T_{\xi}^{(1)} L_{\eta}: L_{\eta}\right| \leqq\left|C_{K_{\eta} / L_{\eta}}\left(Q_{\xi}\right)\right| \leqq 2^{2},
$$

so $\left[Q_{\eta}, O^{2}\left(G_{\eta}\right)\right]$ induces a factorwise quasitransvection on $T_{\xi}^{(1)} / W_{\xi}$ by (3.8). Hence the assertion follows from (2.11)-(1).

(8.20) Let $(\beta, \delta) \in \mathcal{C}_{0}{ }^{\prime}$ and suppose $\left|K_{\beta}: K_{\beta} \cap Q_{\delta-2}\right|=2$. Then either $V_{\delta} \subseteq$ $K_{\beta}$ or $V_{\delta-2} \subseteq U_{\beta}$.

Proof. Take $\alpha, \alpha^{\prime} \in \Delta(\beta)$ so that

$$
W_{\alpha} \cap W_{\beta+1}=W_{\alpha^{\prime}} \cap W_{\beta+1}=W_{\alpha} \cap W_{\alpha^{\prime}}=V_{\beta} .
$$

If $T_{\alpha} \subseteq Q_{\delta-2}$, then, since $G_{\beta}=\left\langle G_{\alpha \beta}, U_{\delta}\right\rangle \not T_{\alpha} \subseteq Q_{\delta-1}, V_{\delta} \subseteq K_{\beta}$ by (8.12)-(1). Thus we may assume that $T_{\alpha} \varsubsetneqq Q_{\delta-2} \nsupseteq T_{\alpha^{\prime}}$. Hence

$$
W_{\alpha} \supseteqq\left[T_{\alpha}, U_{\delta-2}\right] \varsubsetneqq V_{\beta} \nsupseteq\left[T_{\alpha^{\prime}}, U_{\delta-2}\right] \leqq W_{\alpha^{\prime}}
$$

so $\left[T_{\alpha}, U_{\delta-2}\right] \neq\left[T_{\alpha^{\prime}}, U_{\delta-2}\right]$. Since $K_{\beta} Q_{\delta-2}=U_{\theta} Q_{\delta-2}$ for $\theta \in \Delta(\alpha)$ with $U_{\theta} \varsubsetneqq$ $Q_{\delta-2}$, we have $\left|\left[K_{\beta}, U_{\delta-2}\right]\right| \leqq\left|\left[U_{\theta}, U_{\delta-2}\right] V_{\delta-2}\right|=\left|U_{\theta}: U_{\theta} \cap Q_{\delta-2}\right| \cdot\left|V_{\delta-2}\right|=\mid K_{\theta}$ : $K_{\theta} \cap Q_{\delta-2}|\cdot| V_{\delta-2} \mid \leqq 2^{2}$ by (8.11)-(2), so $V_{\delta-2} \subseteq\left[K_{\beta}, U_{\delta-2}\right]=\left[T_{\alpha}, U_{\delta-2}\right]\left[T_{\alpha^{\prime}}, U_{\delta-2}\right]$ $\subseteq W_{\alpha} W_{\alpha^{\prime}}=U_{\beta}$. 
Proposition 8.21 Let $\eta \in \mathcal{B}$. Then $n\left(G_{\eta}, K_{\eta} / U_{\eta}\right)=1$ and $\left(G_{\eta}, K_{\eta} / U_{\eta}\right) \ll$ $\left(O_{4}^{+}(2), G F(2)^{4}\right)$.

Proof. It suffices to prove that $n\left(G_{\eta}, K_{\eta} / U_{\eta}\right)=1$ by (8.18). Assume otherwise.

(1) $\quad K_{\beta} \varsubsetneqq Q_{\delta-2}$ for all $(\beta, \delta) \in \mathcal{C}_{0}{ }^{\prime}$.

Proof. This follows from (8.14) as in the proof of (1) of (8.18).

(2) $\quad K_{\beta} \nsupseteq V_{\delta}$ for all $(\beta, \delta) \in \mathcal{C}_{0}{ }^{\prime}$.

Proof. Suppose $K_{\beta} \supseteq V_{\delta}$ for some $(\beta, \delta) \in \mathcal{C}_{0}{ }^{\prime}$. Since $U_{\delta-2} \cap T_{\delta-1}{ }^{(1)} \neq$ $U_{\delta} \cap T_{\delta-1}{ }^{(1)}$ as in the proof of (7) of (8.18), $G_{\delta-1}=\left\langle G_{\delta-2, \delta-1}, G_{\delta-1, \delta}\right\rangle$ by (8.19) and (3.10)-(2), and so $V_{\delta-1}=V_{\delta-2} V_{\delta}$. Therefore $K_{\beta}$ centralizes $W_{\delta-1}=V_{\delta-2} V_{\delta}\left[U_{\beta}, U_{\delta}\right]$, so $G_{\delta-2} \neq\left\langle K_{\beta}, G_{\delta-2, \delta-1}\right\rangle$. Thus, by (8.18) and (3.10)-(1), there exists $\lambda \in \Delta(\delta-2)$ such that $K_{\beta} \subseteq G_{\delta-2, \lambda}, W_{\lambda}=W_{\delta-1}$, and $T_{\lambda}=T_{\delta-1} \varsubsetneqq Q_{\beta}$. Take $\mu \in \Delta(\lambda)$ so that $U_{\mu} \varsubsetneqq Q_{\beta}$. Since $K_{\beta} \subseteq C_{G_{\delta-2}}\left(W_{\lambda}\right) \subseteq Q_{\lambda}$, we have $n\left(G_{\beta}, K_{\beta} / U_{\beta}\right)=1$ by (8.14), a contradiction.

(3) A contradiction.

Proof. Take $(\beta, \delta) \in \mathcal{C}_{0}{ }^{\prime}, \alpha \in \Delta(\beta)$, and $\theta \in \Delta(\alpha)$ so that $(\theta, \delta-2) \in \mathcal{C}_{0}{ }^{\prime}$.

Suppose $\left|K_{\beta}: K_{\beta} \cap Q_{\delta-2}\right|=2^{2}$, and choose $\lambda \in \Delta(\delta-2)$ so that $W_{\delta-3} \cap W_{\lambda}=$ $V_{\delta-2}$. Then $\left[U_{\beta}, T_{\lambda}\right]=1$ by $(8.15)$, so $V_{\theta} \subseteq K_{\delta-2}$ by $(8.12)-(1)$, which contradicts (2).

Therefore we have $\left|K_{\beta}: K_{\beta} \cap Q_{\delta-2}\right|=2$, and hence either $V_{\delta} \subseteq K_{\beta}$ or $V_{\delta-2}$ $\subseteq U_{\beta} \subseteq K_{\theta}$ by $(8.20)$, which also contradicts (2).

Define $L_{\eta}=\cap T_{\xi}^{G_{\eta}}$ and $N_{\eta}=C_{K_{\eta} \bmod L_{\eta}}\left(O^{2}\left(G_{\eta}\right)\right)$ for $\langle\xi, \eta\rangle \in E(\Gamma)$.

COROLlaRY 8.22 The following holds.

(1) $L_{\eta}=T_{\xi} \cap N_{\eta} \supseteqq\left[K_{\eta}, Q_{\eta}\right]$.

(2) $\quad 2^{4}=\left|K_{\eta} / N_{\eta}\right| \leqq\left|K_{\eta} / L_{\eta}\right| \leqq 2^{6}$.

(3) $\quad T_{\xi}^{(1)} N_{\eta} / N_{\eta}=C_{K_{\eta} / N_{\eta}}\left(G_{\xi \eta}\right)$ and $\left|T_{\xi}^{(1)} N_{\eta} / N_{\eta}\right|=2$.

(4) $T_{\xi} N_{\eta} / N_{\eta}=\left[K_{\eta} / N_{\eta}, E_{\xi \eta}\right]=C_{K_{\eta} / N_{\eta}}\left(E_{\xi \eta}\right)$ and $\left|T_{\xi} N_{\eta} / N_{\eta}\right|=2^{2}$.

(5) $O^{2}\left(G_{\xi} / Q_{\xi} C_{G_{\xi}}\left(T_{\xi}\right)\right) \cong \mathbf{Z}_{3}$.

(6) The following four conditions for $x \in O^{2}\left(G_{\eta}\right)$ are equivalent.

(i) $\quad C_{U_{\eta} / V_{\eta}}(x)=1$.

(ii) $\quad C_{K_{\eta} / U_{\eta}}(x)=1$.

(iii) $W_{\xi} \cap W_{\xi}^{x}=V_{\eta}$.

(iv) $T_{\xi} N_{\eta} \cap T_{\xi}^{x} N_{\eta}=N_{\eta}$.

(7) Let $x \in O^{2}\left(G_{\eta}\right)$. If $C_{K_{\eta} / U_{\eta}}(x)=1$, then $K_{\eta}=\left\langle T_{\xi}\langle x\rangle\right.$.

Proof. Part (1) follows from (8.21), (2.14)-(1), and the definition of $L_{\eta}$ and 
$N_{\eta}$, and then (8.21) and (2.4)-(3) show that $\left|K_{\eta} / N_{\eta}\right|=2^{4}$. Since $T_{\xi}^{(2)} \subseteq W_{\xi} \subseteq$ $L_{\eta} \nsupseteq T_{\xi}^{(1)}$ by (8.13), (8.4), and (3.8), $T_{\xi}^{(1)} N_{\eta} / N_{\eta}=C_{K_{\eta} / N_{\eta}}\left(G_{\xi \eta}\right),\left|T_{\xi}^{(1)} N_{\eta} / N_{\eta}\right|=$ 2 , and $\left|T_{\xi} / L_{\eta}\right|=\left|T_{\xi} N_{\eta} / N_{\eta}\right|=2^{2}$ by $(2.5)-(3)$. Therefore $\left|K_{\eta} / L_{\eta}\right| \leqq 2^{4} \cdot\left|T_{\xi} / L_{\eta}\right|=$ $2^{6}$ by $(3.9)-(1)$. Since $E_{\xi \eta}=C_{Q_{\xi}}\left(W_{\xi}\right) Q_{\eta}$ and $\left[T_{\xi}, C_{Q_{\xi}}\left(W_{\xi}\right)\right] \subseteq W_{\xi} \cong N_{\eta}$ by (8.5), (2.5)-(4) shows that $T_{\xi} N_{\eta} / N_{\eta}=\left[K_{\eta} / N_{\eta}, E_{\xi \eta}\right]=C_{K_{\eta} / N_{\eta}}\left(E_{\xi \eta}\right)$, proving (2)-(4).

Since $\left|T_{\xi}: T_{\xi}^{(1)} L_{\eta}\right|=\left|T_{\xi}^{(1)} U_{\eta}: T_{\xi}^{(1)}\right|=2$ and $\left[L_{\eta},\left[Q_{\eta}, O^{2}\left(G_{\eta}\right)\right]\right] \subseteq U_{\eta}$, $\left[Q_{\eta}, O^{2}\left(G_{\eta}\right)\right]$ induces a quasitransvection on $T_{\xi} / T_{\xi}^{(1)}$, and hence $O^{2}\left(G_{\xi} / C_{G_{\xi}}\left(T_{\xi} / T_{\xi}^{(1)}\right)\right) \cong \mathbf{Z}_{3}$. Thus, together with (8.19) and (8.2)-(1), we have $O^{2}\left(G_{\xi} / Q_{\xi} C_{G_{\xi}}\left(T_{\xi}\right)\right) \cong \mathbf{Z}_{3}$, so (5) holds.

Now, let $x \in O^{2}\left(G_{\eta}\right)$. Define $D=C_{G_{\eta}}\left(U_{\eta} / V_{\eta}\right)=C_{G_{\eta}}\left(K_{\eta} / U_{\eta}\right)$ and $H=$ $E_{\xi \eta} O^{2}\left(G_{\eta}\right)$. Then any of the four conditions in (6) is equivalent to the condition that $\langle x\rangle D \Varangle H$ by (2.5)-(11), so (6) follows. Suppose $C_{K_{\eta} / N_{\eta}}(x)=1$, and let $M=$ $\left\langle T_{\xi}^{\langle x\rangle}\right\rangle$. Since $K_{\eta}=M N_{\eta}$ and $\langle x\rangle D \triangleleft O^{2}\left(G_{\eta}\right) D,\left[K_{\eta}, O^{2}\left(G_{\eta}\right)\right] L_{\eta}=\left[K_{\eta}, x\right] L_{\eta} \subseteq$ $M$, so $K_{\eta}=T_{\xi}\left[K_{\eta}, O^{2}\left(G_{\eta}\right)\right]=M$, proving (7).

We will now derive a final contradiction.

\section{There exists $(\beta, \delta) \in \mathcal{C}_{0}{ }^{\prime}$ such that}

$$
\begin{aligned}
& W_{\beta+1} \cap W_{\beta+3}=V_{\beta+2}, W_{\delta-3} \cap W_{\delta-1}=V_{\delta-2}, \quad \text { and } \\
& \text { either }\left[K_{\beta}, U_{\delta-2}\right]=1 \quad \text { or }\left[K_{\delta}, U_{\beta+2}\right]=1 .
\end{aligned}
$$

Proof. Let $(\beta, \delta) \in \mathcal{C}_{0}{ }^{\prime}$. Since $K_{\beta+2} \nsubseteq Q_{\delta}$, there exists $\xi \in \Delta(\beta+2)$ such that $T_{\xi} \nsubseteq Q_{\delta}$ and $W_{\xi} \cap W_{\beta+3}=V_{\beta+2}$ by $(8.22)-(6)(7)$. Take $\eta \in \Delta(\xi)$ so that $U_{\eta} \nsubseteq Q_{\delta}$. Then $(\eta, \delta) \in \mathcal{C}_{0}{ }^{\prime}$. Similarly, we can take $\lambda \in \Delta(\delta-2)$ and $\mu \in \Delta(\lambda)$ such that $W_{\delta-3} \cap W_{\lambda}=V_{\delta-2}$ and $(\eta, \mu) \in \mathcal{C}_{0}{ }^{\prime}$.

Changing notation, let $(\beta, \delta) \in \mathcal{C}_{0}^{\prime}$ so that $W_{\beta+1} \cap W_{\beta+3}=V_{\beta+2}$ and $W_{\delta-3} \cap$ $W_{\delta-1}=V_{\delta-2}$. Suppose

$$
\left[K_{\beta}, U_{\delta-2}\right] \neq 1 \neq\left[K_{\delta}, U_{\beta+2}\right]
$$

Since $K_{\delta} \nsubseteq Q_{\beta+2}$ by (8.11)-(1), we can take, as above, $\lambda \in \Delta(\delta)$ and $\mu \in \Delta(\lambda)$ so that $W_{\delta-1} \cap W_{\lambda}=V_{\delta}$ and $(\beta+2, \mu) \in \mathcal{C}_{0}{ }^{\prime}$. Therefore $G_{\delta-2}=\left\langle G_{\delta-3, \delta-2}, G_{\delta-2, \delta-1}\right\rangle=$ $\left\langle K_{\beta}, Q_{\delta-1}\right\rangle$ by $(8.9)-(1)$ because $Q_{\delta-2} \nsupseteq K_{\beta} \leqq E_{\delta-3, \delta-2}$. Note that $V_{\delta-1}=V_{\delta-2} V_{\delta}$ by (3.10)-(2) and (8.22)-(5) as $U_{\delta-2} \neq U_{\delta}$. If $V_{\delta} \subseteq K_{\beta}$, then $\left[K_{\beta}, V_{\delta-1}\right]=1$, so $\left[G_{\delta-2}, V_{\delta-1}\right]=\left[\left\langle K_{\beta}, Q_{\delta-1}\right\rangle, V_{\delta-1}\right]=1$, a contradiction. Thus $V_{\delta} \nsubseteq K_{\beta}$. If $\left[U_{\beta+2}, U_{\mu}\right]$ $\subseteq W_{\delta-1}$, then $\left[U_{\delta+2}, U_{\mu}\right] \subseteq W_{\delta-1} \cap W_{\lambda}=V_{\delta} \subseteq V_{\lambda}$, which contradicts (2.5)-(9). Thus $\left|U_{\delta}: W_{\delta-1}\left[U_{\beta+2}, U_{\mu}\right]\right| \leqq 2$. Since $K_{\beta+2}$ centralizes $W_{\delta-1}\left[U_{\beta+2}, U_{\mu}\right]$, we have $\left|K_{\beta+2}: K_{\beta+2} \cap Q_{\delta}\right| \leqq 2$, and hence $K_{\beta+2} \subseteq Q_{\delta}$ by $(8.20)$ applied to $(\beta+2, \mu) \in \mathcal{C}_{0}{ }^{\prime}$. Therefore $\left[K_{\beta+2}, U_{\delta}\right]=1$ by $(8.11)-(1)$. 
Now, let $(\beta, \delta) \in \mathcal{C}_{0}{ }^{\prime}$ so that

$W_{\beta+1} \cap W_{\beta+3}=V_{\beta+2}, W_{\delta-3} \cap W_{\delta-1}=V_{\delta-2}, \quad$ and $\quad\left[K_{\beta}, U_{\delta-2}\right]=1$.

(2) $T_{\beta+1} \nsupseteq V_{\delta} \leqq K_{\beta}$.

Proof. By (8.12)-(1), we have $V_{\delta} \subseteq K_{\beta}$. Suppose $V_{\delta} \subseteq T_{\beta+1}$. Then $\left[K_{\beta}, U_{\delta}\right] \subseteq\left[U_{\beta}, U_{\delta}\right]\left[K_{\beta}, U_{\delta} \cap Q_{\beta}\right] V_{\delta} \subseteq T_{\beta+1}$, and so $\left[K_{\beta}, E_{\beta, \beta+1}\right] \subseteq$ $\left[K_{\beta},\left\langle U_{\delta}{ }^{G_{\beta, \beta+1}}\right\rangle Q_{\beta}\right] \subseteq T_{\beta+1}$ by (8.11)-(4), (8.21), and (3.14)-(2). Thus $K_{\beta+2}=$ $T_{\beta+1} T_{\beta+3}=T_{\lambda} T_{\beta+3}$ for $\lambda \in \Delta(\beta+2)$ with $W_{\beta+1} \cap W_{\lambda}=W_{\lambda} \cap W_{\beta+3}=V_{\beta+2}$ because $\left\langle E_{\beta+1, \beta+2}, E_{\beta+2, \beta+3}\right\rangle \supseteqq O^{2}\left(G_{\beta+2}\right) \subseteq\left\langle E_{\lambda, \beta+2}, E_{\beta+2, \beta+3}\right\rangle$ by (2.5)-(11). Therefore $\left[U_{\beta}, U_{\delta}\right] \subseteq\left[K_{\beta+2}, U_{\delta}\right]=\left[T_{\beta+1}, U_{\delta}\right]=\left[T_{\lambda}, U_{\delta}\right] \subseteq W_{\beta+1} \cap W_{\lambda}=V_{\beta+2}$ $\subseteq V_{\beta+1}$, so $\left[U_{\beta}, U_{\delta}\right]=1$, a contradiction.

(3) $\left[U_{\beta}, K_{\delta-2} \cap Q_{\beta}\right]=1$.

Proof. Suppose false. Then $V_{\beta}=\left[U_{\beta}, K_{\delta-2} \cap Q_{\beta}\right] \subseteq U_{\delta-2} \subseteq T_{\delta-1}$. Thus $\left[K_{\delta}, V_{\beta+1}\right]=\left[K_{\delta}, V_{\beta} V_{\beta+2}\right]=1$, and so $K_{\delta} \subseteq Q_{\beta+2}$ by $(8.9)-(1)$, and then $\left[K_{\delta}, U_{\beta+2}\right]=1$ by $(8.11)-(1)$. However, applying $(2)$ to $(\delta, \beta) \in \mathcal{C}_{0}{ }^{\prime}$, we have $V_{\beta} \nsubseteq$ $T_{\delta-1}$, a contradiction.

(4) Let $\lambda \in \Delta(\delta-2)$, and assume $T_{\lambda} \nsubseteq Q_{\beta}$. Then

$$
\left[K_{\beta}, T_{\lambda}\right] U_{\beta}=U_{\beta} W_{\lambda} \quad \text { and } \quad\left|U_{\beta} W_{\lambda}: U_{\beta}\right|=2 \text {. }
$$

Proof. Let $\mu, \mu^{\prime} \in \Delta(\lambda)$ so that $T_{\lambda}=\left\langle U_{\delta-2}, U_{\mu}, U_{\mu^{\prime}}\right\rangle$. Since $\left[K_{\delta-2}, Q_{\delta-2}\right]$ $\subseteq L_{\delta-2} \subseteq T_{\lambda} \cap Q_{\beta}$, and $U_{\mu}$ and $U_{\mu^{\prime}}$ are conjugate in $Q_{\delta-2}, U_{\mu} \nsubseteq Q_{\beta} \nsupseteq U_{\mu^{\prime}}$. Thus $T_{\lambda}=\left(T_{\lambda} \cap Q_{\beta}\right) U_{\mu}=\left(T_{\lambda} \cap Q_{\beta}\right) U_{\mu^{\prime}}$ by $(8.16)$, so

$$
\left[K_{\beta}, T_{\lambda}\right] U_{\beta}=\left[K_{\beta}, U_{\mu}\right] U_{\beta}=\left[K_{\beta}, U_{\mu^{\prime}}\right] U_{\beta}
$$

by (3) and (8.5). Therefore,

$$
\begin{aligned}
& {\left[K_{\beta}, U_{\mu}\right] U_{\beta}=U_{\beta}\left[U_{\beta}, U_{\mu}\right]\left[K_{\beta}, U_{\mu} \cap Q_{\beta}\right] V_{\mu}=U_{\beta} V_{\mu} \text { and, similarly, }} \\
& {\left[K_{\beta}, U_{\mu^{\prime}}\right] U_{\beta}=U_{\beta} V_{\mu^{\prime}} .}
\end{aligned}
$$

Hence $\left[K_{\beta}, T_{\lambda}\right] U_{\beta}=U_{\beta} V_{\mu} V_{\mu^{\prime}}=U_{\beta}\left[U_{\beta}, U_{\mu}\right] V_{\lambda}=U_{\beta} W_{\lambda}$ by (8.9)-(1) and $\mid U_{\beta} W_{\lambda}$ : $U_{\beta}|=| U_{\beta} V_{\mu}: U_{\beta} \mid=2$.

(5) Let $\lambda_{1}, \lambda_{2}, \lambda_{3} \in \Delta(\delta-2)$ so that

$$
W_{\lambda_{i}} \cap W_{\lambda_{j}}=V_{\delta-2} \quad(i \neq j) .
$$

Then precisely two of $T_{\lambda_{1}}, T_{\lambda_{2}}, T_{\lambda_{3}}$ are contained in $Q_{\beta}$. In particular, $\mid K_{\delta-2}$ : $K_{\delta-2} \cap Q_{\beta} \mid=2$. 
Proof. $\quad$ Note that $K_{\delta-2}=\left\langle T_{\lambda_{1}}, T_{\lambda_{2}}, T_{\lambda_{3}}\right\rangle$, and suppose $T_{\lambda_{1}} \nsubseteq Q_{\beta} \nsupseteq T_{\lambda_{2}}$. We may assume that $K_{\delta-2}=\left(K_{\delta-2} \cap Q_{\beta}\right) T_{\lambda_{1}} T_{\lambda_{2}}$. Hence we have

$$
U_{\beta} U_{\delta-2} \supseteq\left[K_{\beta}, K_{\delta-2}\right] U_{\beta} \supseteq\left[K_{\beta}, T_{\lambda_{1}}\right]\left[K_{\beta}, T_{\lambda_{2}}\right] U_{\beta}=U_{\beta} W_{\lambda_{1}} W_{\lambda_{2}}=U_{\beta} U_{\delta-2}
$$

by (3).

If $\left|K_{\delta-2}: K_{\delta-2} \cap Q_{\beta}\right|=2^{2}$, then $\left|\left[K_{\beta}, K_{\delta-2}\right] U_{\beta}: U_{\beta}\right| \leqq 2^{2}$ by (4), so $\left|U_{\beta}: U_{\beta} \cap U_{\delta-2}\right|=\left|U_{\beta} U_{\delta-2}: U_{\beta}\right| \leqq 2^{2}$. If $\left|K_{\delta-2}: K_{\delta-2} \cap Q_{\beta}\right|=2$, then $\left|\left[K_{\beta}, K_{\delta-2}\right] U_{\beta}: U_{\beta}\right| \leqq 2$ by (3) and (4) as $K_{\delta-2}=\left(K_{\delta-2} \cap Q_{\beta}\right) T_{\lambda_{1}}$, so $\left|U_{\beta}: U_{\beta} \cap U_{\delta-2}\right|=\left|U_{\beta} U_{\delta-2}: U_{\beta}\right| \leqq 2$. Therefore $\left|U_{\beta} / V_{\beta}: C_{U_{\beta} / V_{\beta}}\left(K_{\delta-2}\right)\right| \leqq$ $\left|U_{\beta}: U_{\beta} \cap Q_{\delta-2}\right| \leqq\left|K_{\delta-2}: K_{\delta-2} \cap Q_{\beta}\right|$, so $V_{\beta} \subseteq W_{\beta+1} \subseteq C_{U_{\beta}}\left(K_{\delta-2}\right)=U_{\beta} \cap U_{\delta-2}$ $\subseteq T_{\delta-1}$ by $(2.5)-(5)$, which is a contradiction as in (3).

Now, choose $\lambda \in \Delta(\delta-2)$ so that

$$
W_{\delta-3} \cap W_{\lambda}=W_{\lambda} \cap W_{\delta-1}=W_{\delta-3} \cap W_{\delta-1}=V_{\delta-2} .
$$

Then, since $\left|T_{\delta-1}: T_{\delta-1} \cap Q_{\beta}\right|=2$ by (8.16), we have $T_{\lambda} \subseteq Q_{\beta}$, so $\mid K_{\delta-2}$ : $K_{\delta-2} \cap Q_{\beta} \mid=2$.

$$
\left|K_{\delta-2}: L_{\delta-2}\right|=2^{6} \text { and }\left[N_{\delta-2}, G_{\delta-2}\right] L_{\delta-2} \nsubseteq Q_{\beta} .
$$

Proof. Suppose false. Let $H=K_{\delta-2} \cap Q_{\beta}$ and $\widetilde{K}_{\delta-2}=$ $K_{\delta-2} /\left[N_{\delta-2}, G_{\delta-2}\right] L_{\delta-2}$. Then $\left|\widetilde{K}_{\delta-2}\right| \leqq 2^{5}$ and $\left|\widetilde{K}_{\delta-2}: \widetilde{H}\right|=2$. We see that this contradicts (5), applying (2.6) for $\widetilde{K}_{\delta-2}$ and $\widetilde{H}$.

Let $M_{\xi}=\cap K_{\eta}{ }^{G_{\xi}}$ and $F_{\xi}=\left\langle K_{\eta}{ }^{G_{\xi}}\right\rangle$ for $\langle\xi, \eta\rangle \in E(\Gamma)$.

(7) $\quad M_{\xi}=T_{\xi}$ for $\xi \in \mathcal{A}$.

Proof. We may assume $\xi=\delta-3$. Suppose $M_{\delta-3} \neq T_{\delta-3}$. Then $\mid M_{\delta-3}$ : $T_{\delta-3} \mid=2$ by $(3.9)-(3)$, and hence $\left[N_{\delta-2}, G_{\delta-2}\right] L_{\delta-2}=M_{\delta-3} \cap N_{\delta-2} \subseteq Q_{\beta}$, which contradicts $(6)$.

(8) A contradiction.

Proof. Let $\eta \in \Delta(\beta+1)$ so that $U_{\beta} \neq U_{\eta} \neq U_{\beta+2}$. Since $U_{\beta}$ and $U_{\eta}$ are conjugate in $Q_{\beta+2}$ and $\left[K_{\beta+2}, Q_{\beta+2}\right] \subseteq L_{\beta+2} \subseteq Q_{\delta}$, we have $U_{\eta} \varsubsetneqq Q_{\delta}$, and, moreover, $T_{\beta+1}=K_{\beta} \cap K_{\beta+2}=K_{\beta} \cap K_{\eta}$ by (7) and (8.22)-(1)(5).

Suppose $b=7$. Then $T_{\beta+1} \nsupseteq V_{\delta} \subseteq K_{\beta} \cap K_{\beta+2}=T_{\beta+1}$ by (2), a contradiction.

Suppose $b \geqq 9$. Then $F_{\beta+1}$ is abelian, so $\left[K_{\eta}, V_{\delta}\right] \subseteq\left[F_{\beta+1}, V_{\delta}\right]=1$. Thus $\left[K_{\eta}, U_{\delta-2}\right]=1$ by $(8.9)-(1)$ and (8.11)-(1). Therefore, applying $(2)$ for $(\eta, \delta) \in \mathcal{C}_{0}{ }^{\prime}$, we have $V_{\delta} \subseteq K_{\eta}$, so $T_{\beta+1} \varsubsetneqq V_{\delta} \subseteq K_{\beta} \cap K_{\eta}=T_{\beta+1}$, also a contradiction.

Acknowledgement. I would like to express my thanks to Professor Kensaku Gomi for his valuable advices and for showing me his unpublished results in Section 3 and in the second case of Section 7. 


\title{
References
}

[1] M. Aschbacher, $G F(2)$-representation of finite groups, Amer. J. Math., 104 (1982), 683771 .

[2] A. Delgado, D.M. Goldshmidt, and B. Stellmacher, Groups and Graphs: New Results and Methods, Birkhäuser Verlag, Basel, Boston, Stuttgart, 1985.

[3] K. Gomi, A note on the thin finite simple groups, J. Algebra, 102 (1986), 194-198.

[4] K. Gomi, A pushing up theorem for groups of characteristic 2 type, Sci. Papers College Arts Sci. Univ. Tokyo, 37 (1987), 73-102.

[ 5 ] K. Gomi, Characteristic pairs for 2-groups, J. Algebra, 94 (1985), 488-510.

[6] K. Gomi and M. Hayashi, A pushing-up approach to the quasithin simple finite groups with solvable 2-local subgroups, preprint.

[ 7 ] K. Gomi and Y. Tanaka, On pairs of groups having a common 2-subgroup of odd indices I-II, Sci. Papers College Arts Sci. Univ. Tokyo, 35 (1985), 11-30; 40 (1990), 37-47.

[8] D. Gorenstein and R. Lyons, Nonsolvable finite groups with solvable 2-local subgroups, J. Algebra, 38 (1976), 453-522.

[ 9 ] Z. Janko, Nonsolvable finite groups all of whose 2-local subgroups are solvable, J. Algebra, 21 (1972), 458-517.

[10] F. Smith, Finite simple groups all of whose 2-local subgroups are solvable, J. Algebra, 34 (1975), 481-520.

[11] Y. Tanaka, Certain pairs of finite groups with a common 2-subgroup, J. Fac. Sci. Univ. Tokyo Sect. IA Math., 33 (1986), 247-272.

[12] J.G. Thompson, Nonsolvable finite groups all of whose local subgroups are solvable I-VI, Bull. Amer. Math. Soc., 74 (1968), 387-483; Pacific J. Math., 33 (1970), 431-536; 39 (1971), 483-534; 48 (1973), 511-592; 50 (1974), 215-297; 51 (1974), 573-630.

\author{
Department of Mathematics, \\ FaCUlty of SCience \\ UNIVERSITY OF TOKYO, \\ HONGO, TOKYO, 113 JAPAN
}

